\title{
TWO-DIMENSIONAL NONLOCAL CAHN-HILLIARD-NAVIER-STOKES SYSTEMS WITH VARIABLE VISCOSITY, DEGENERATE MOBILITY AND SINGULAR POTENTIAL
}

\author{
S. FRIGERI, C.G. GAL, M. GRASSELLI, AND J. SPREKELS
}

\begin{abstract}
We consider a nonlinear system which consists of the incompressible Navier-Stokes equations coupled with a convective nonlocal Cahn-Hilliard equation. This is a diffuse interface model which describes the motion of an incompressible isothermal mixture of two (partially) immiscible fluids having the same density. We assume that both the viscosity and mobility functions depend smoothly on the order parameter. Moreover, we assume that the mobility degenerates at the pure phases and that the potential is singular (e.g. of logarithmic type). This system is endowed with a no-slip boundary condition for the (average) velocity and a homogeneous Neumann boundary condition for the chemical potential. Thus the total mass is conserved. In the two-dimensional case, this problem was already analyzed in some joint papers of the first three authors. However, in the present general case, only the existence of a global weak solution, the (conditional) weak-strong uniqueness and the existence of the global attractor were proven. Here we are able to establish the existence of a (unique) strong solution through an approximation procedure based on time discretization. As a consequence, we can prove suitable uniform estimates which allow us to show some smoothness of the global attractor. Finally, we discuss the existence of strong solutions for the convective nonlocal Cahn-Hilliard equation, with a given velocity field, in the three dimensional case as well.
\end{abstract}

\section{INTRODUCTION}

The so-called model $\mathbf{H}$ (see, for instance, [36] and references therein) has been proposed to describe the motion of a binary mixture of two isothermal, partially immiscible and incompressible fluids. This model is based on the diffuse interface approach and leads to the formulation of a Cahn-Hilliard-Navier-Stokes (CHNS) system for the average velocity $\boldsymbol{u}$ and the order parameter $\varphi$ (i.e., the relative concentration of one of the fluid components). In the case of matched constant densities, a rather general CHNS system is the following:

$$
\begin{aligned}
& \boldsymbol{u}_{t}-2 \operatorname{div}(\nu(\varphi) D \boldsymbol{u})+(\boldsymbol{u} \cdot \nabla) \boldsymbol{u}+\nabla \pi=\mu \nabla \varphi+\boldsymbol{v}, \\
& \varphi_{t}+\boldsymbol{u} \cdot \nabla \varphi=\operatorname{div}(m(\varphi) \nabla \mu), \\
& \mu=-K * \varphi+F^{\prime}(\varphi), \\
& \operatorname{div}(\boldsymbol{u})=0
\end{aligned}
$$

in $\Omega \times(0, T)$, where $\Omega \subset \mathbb{R}^{d}, d=2,3$, is a bounded smooth domain (say, e.g., of class $\left.\mathcal{C}^{2}\right), T>0$ is a prescribed final time, $\nu$ stands for the fluid viscosity, $D$ denotes the symmetric gradient, that is, $D \boldsymbol{u}:=\left(\nabla \boldsymbol{u}+\nabla^{T} \boldsymbol{u}\right) / 2$ and $\boldsymbol{v}$ is a given external force (the density has been taken equal to one). The Cahn-Hilliard $(\mathrm{CH})$ equation (1.2) with mobility $m$ is characterized by a nonlocal chemical potential (1.3) where $K: \mathbb{R}^{d} \rightarrow \mathbb{R}$ is a (sufficiently) smooth even interaction kernel (see [33], cf. also $[7,32,34]$ and the discussion in [35]).

2010 Mathematics Subject Classification. 35Q30, 37L30, 45K05, 76D03, 76T99.

Key words and phrases. Incompressible binary fluids, Navier-Stokes equations, nonlocal Cahn-Hilliard equations, time discretization schemes, strong solutions, regularization, global attractors. 
System (1.1)-(1.4) is subject to the no-slip boundary condition for the velocity $\boldsymbol{u}$ and to the homogeneous Neumann boundary condition for the chemical potential $\mu$ (which ensures the conservation of the total mass), namely,

$$
\boldsymbol{u}=\mathbf{0}, \quad m(\varphi) \nabla \mu \cdot \boldsymbol{n}=0,
$$

on $\partial \Omega \times(0, T)$. Initial conditions must also be prescribed,

$$
\boldsymbol{u}(0)=\boldsymbol{u}_{0}, \quad \varphi(0)=\varphi_{0}, \text { in } \Omega .
$$

Here, $\boldsymbol{n}$ stands for the outward normal to the boundary $\partial \Omega$ of $\Omega$, while $\boldsymbol{u}_{0}$ and $\varphi_{0}$ are given.

Problem (1.1)-(1.6) has been studied so far under various assumptions on $\nu, m$ and $F$ (see $[14,20,21,22,23,24,25]$, cf. also [19] for unmatched densities and [26] for inviscid fluids). The model studied in these papers is based on a more, say, phenomenological free energy functional (see [8]), which leads to the chemical potential

$$
\mu=-K * \varphi+a(x) \varphi+F^{\prime}(\varphi)
$$

where $a(x)=\int_{\Omega} K(x-y) d y$ and $F$ is some (possibly singular) double-well potential. However, from the mathematical viewpoint all the results obtained so far also hold in the present case. Actually, proofs are simpler and assumptions on $K$ are more general (see the discussion in [35, Introduction], cf. also Remark 2.6 below).

On the other hand, the physically more relevant case, namely, when the viscosity depends on $\varphi$, the mobility $m$ degenerates at pure phases (i.e. $\varphi= \pm 1$ ) and $F$ is a singular potential (say, of logarithmic type) is still substantially open. Concerning $m$ and $F$, we recall that a physically relevant choice is

$$
\begin{aligned}
& m(s)=m_{0}\left(1-s^{2}\right), \\
& F(s)=\frac{\theta}{2}((1+s) \log (1+s)+(1-s) \log (1-s)),
\end{aligned}
$$

where $s \in(-1,1), m_{0}>0$ and $\theta>0$. In this case, the existence of weak solutions $(d=2,3)$ was proved in [24], where, for simplicity, the viscosity $\nu$ was assumed to be constant (as far as existence of weak solutions is concerned, the case of a $\nu$ depending on $\varphi$ can be dealt without difficulties as well).

It is worth recalling that for CHNS systems, where the $\mathrm{CH}$ equation is the standard (local) one (see, for instance, $[1,2,12,13,27,28,29,37,44,47]$ ), the case of degenerate mobility and singular potential is already difficult in the case of the $\mathrm{CH}$ alone (cf. [16]). More precisely, the existence of a weak solution is essentially the only available result, as far as we know (see [12], and see also [3] for the unmatched densities case).

Going back to our nonlocal system, in the two-dimensional case, the existence of the global attractor has also been proved in [24] for constant viscosity. This result can be extended to the case of $\nu$ depending on $\varphi$ as well.

Uniqueness of weak solutions is a more delicate issue. Indeed, it has been established in [20] for the case of constant viscosity only (incidentally, this entails the connectedness of the global attractor). If the viscosity depends on $\varphi$, then weak-strong uniqueness holds true for constant mobility and regular potential (i.e., defined on $\mathbb{R}$ ) with polynomially controlled growth, but in the more general case ( $m$ degenerate and $F$ singular) only a conditional weak-strong uniqueness has been proven by assuming the existence of a strong solution (see [20]). Therefore, in the twodimensional case an open issue is the existence of a strong solution under the aforementioned assumptions on $\nu, m$ and $F$. This is precisely the goal of the present contribution.

Proving the existence of strong solutions when $\nu$ depends on $\varphi$ is much more difficult with respect to the case of a constant $\nu$ (cf. [20], cf. also Remark 4.4 below). We recall that in the simplest case 
(i.e., $\nu$ and $m$ are constants and $F$ is regular), existence of strong solutions in two dimensions was proven in [23].

The existence of a strong solution to (1.1)-(1.6) paves the road for two further results. The first is concerned with uniform in time regularization estimates, which, in particular, entails some smoothness of the global attractor. The second is concerned with the convective nonlocal $\mathrm{CH}$ equation, for which we are able to prove the existence of strong solutions in dimension three under quite general regularity assumptions on the given velocity field. In particular, this allows us to deduce, as above, further regularity of the global attractor.

The plan of the paper is as follows: in the next section, besides some notation and definitions, the known results on existence and uniqueness of weak solutions are reported for the sake of convenience. Section 3 is devoted to state the main regularity result of the paper whose proof is given in Section 4. Section 5 contains uniform in time estimates and the related regularity of the global attractor. In the final Section 6, we extend the analysis of the previous sections to the convective nonlocal $\mathrm{CH}$ equation with a given velocity field.

\section{WEAK SOLUTIONS: WHAT IS KNOWN}

Let us fix some notation first. We set $H:=L^{2}(\Omega), V:=H^{1}(\Omega)$, and, for $d=2,3$, we introduce the function spaces

$$
L_{\text {div }}^{r}(\Omega)^{d}:={\overline{\left\{\boldsymbol{u} \in C_{0}^{\infty}(\Omega)^{d}: \operatorname{div}(\boldsymbol{u})=0\right\}}}^{L^{r}(\Omega)^{d}}, \quad 2 \leq r \leq \infty,
$$

and

$$
V_{d i v}:=\left\{\boldsymbol{u} \in H_{0}^{1}(\Omega)^{d}: \operatorname{div}(\boldsymbol{u})=0\right\} .
$$

We also define $G_{d i v}:=L_{d i v}^{2}(\Omega)^{d}$. Recall that $G_{d i v}$ and $V_{d i v}$ are the classical Hilbert spaces for the incompressible Navier-Stokes equations with no-slip boundary conditions (see, e.g., [12, 46]). Denote by $\|\cdot\|$ and $(\cdot, \cdot)$ the norm and the scalar product, respectively, on both $H$ and $G_{d i v}$, as well as on $L^{2}(\Omega)^{d}$ and $L^{2}(\Omega)^{d \times d}$. The notation $\langle\cdot, \cdot\rangle_{X}$ and $\|\cdot\|_{X}$ will stand for the duality pairing between a Banach space $X$ and its dual $X^{\prime}$, and for the norm of $X$, respectively. For every $f \in V^{\prime}$, we set $\bar{f}:=|\Omega|^{-1}\langle f, 1\rangle_{V}$. Here, $|\Omega|$ is the Lebesgue measure of $\Omega$. The Hilbert space $V_{\text {div }}$ is endowed with the scalar product

$$
(\boldsymbol{u}, \boldsymbol{v})_{V_{d i v}}=(\nabla \boldsymbol{u}, \nabla \boldsymbol{v})=2(D \boldsymbol{u}, D \boldsymbol{v}), \quad \forall \boldsymbol{u}, \boldsymbol{v} \in V_{\text {div }} .
$$

Let us recall the definition of the Stokes operator $S: D(S) \cap G_{d i v} \rightarrow G_{d i v}$ in the case of the no-slip boundary condition (1.5) 1 , i.e., $S=-P \Delta$ with domain $D(S)=H^{2}(\Omega)^{d} \cap V_{d i v}$, where $P: L^{2}(\Omega)^{d} \rightarrow G_{d i v}$ is the Leray projector (see, for instance, $[12,46]$ ). Notice that we have

$$
(S \boldsymbol{u}, \boldsymbol{v})=(\boldsymbol{u}, \boldsymbol{v})_{V_{\text {div }}}=(\nabla \boldsymbol{u}, \nabla \boldsymbol{v}), \quad \forall \boldsymbol{u} \in D(S), \quad \forall \boldsymbol{v} \in V_{\text {div }}
$$

It is well known that $S^{-1}: G_{d i v} \rightarrow G_{d i v}$ is a self-adjoint compact operator in $G_{d i v}$ and by the classical spectral theorems there exists a sequence $\lambda_{j}$ with $0<\lambda_{1} \leq \lambda_{2} \leq \cdots$ and $\lambda_{j} \rightarrow \infty$, and a family of $\boldsymbol{w}_{j} \in D(S)$ which is orthonormal in $G_{d i v}$ and such that $S \boldsymbol{w}_{j}=\lambda_{j} \boldsymbol{w}_{j}$.

We also need to recall Poincaré's inequality

$$
\lambda_{1}\|\boldsymbol{u}\|^{2} \leq\|\nabla \boldsymbol{u}\|^{2}, \quad \forall \boldsymbol{u} \in V_{\text {div }},
$$

and other two inequalities, which are valid in dimension two and will be used repeatedly in the course of our analysis. More precisely, the particular case of the Gagliardo-Nirenberg inequality (see, e.g., [10])

$$
\|v\|_{L^{2 q}(\Omega)} \leq \widehat{C}_{2}\|v\|^{1 / q}\|v\|_{V}^{1-1 / q}, \quad \forall v \in V, \quad 2 \leq q<\infty
$$


as well as Agmon's inequality (see [5])

$$
\|v\|_{L^{\infty}(\Omega)} \leq \widehat{C}_{3}\|v\|^{1 / 2}\|v\|_{H^{2}(\Omega)}^{1 / 2}, \quad \forall v \in H^{2}(\Omega) .
$$

In these inequalities, the positive constant $\widehat{C}_{2}$ depends on $q$ and on $\Omega \subset \mathbb{R}^{2}$, while the positive constant $\widehat{C}_{3}$ depends on $\Omega$ only.

The trilinear form $b$ appearing in the weak formulation of the Navier-Stokes equations is the usual one, namely,

$$
b(\boldsymbol{u}, \boldsymbol{v}, \boldsymbol{w}):=\int_{\Omega}(\boldsymbol{u} \cdot \nabla) \boldsymbol{v} \cdot \boldsymbol{w} d x, \quad \forall \boldsymbol{u}, \boldsymbol{v}, \boldsymbol{w} \in V_{\text {div }} .
$$

The associated bilinear operator $\mathcal{B}$ from $V_{\text {div }} \times V_{\text {div }}$ into $V_{\text {div }}^{\prime}$ is defined by $\langle\mathcal{B}(\boldsymbol{u}, \boldsymbol{v}), \boldsymbol{w}\rangle:=b(\boldsymbol{u}, \boldsymbol{v}, \boldsymbol{w})$, for all $\boldsymbol{u}, \boldsymbol{v}, \boldsymbol{w} \in V_{\text {div }}$. We also set $\mathcal{B} \boldsymbol{u}:=\mathcal{B}(\boldsymbol{u}, \boldsymbol{u})$, for every $\boldsymbol{u} \in V_{\text {div }}$.

If $X$ is a (real) Banach space, we shall denote by $L_{t b}^{p}(0, \infty ; X), 1 \leq p<\infty$, the space of functions $f \in L_{l o c}^{p}([0, \infty) ; X)$ that are translation bounded in $L_{l o c}^{p}([0, \infty) ; X)$, i.e., such that

$$
\|f\|_{L_{t b}^{p}(0, \infty ; X)}^{p}:=\sup _{t \geq 0} \int_{t}^{t+1}\|f(s)\|_{X}^{p} d s<\infty .
$$

We are now ready to recall the result on the existence of weak solutions proven in [24]. For completeness, we deal with $d=2$ and $d=3$. The assumptions on the kernel $K$, on the viscosity $\nu$ are the following.

$(\mathbf{K}): K(\cdot-x) \in W^{1,1}(\Omega)$ for almost any $x \in \Omega$, and $K$ satisfies $K(x)=K(-x)$ and

$$
a^{*}:=\sup _{x \in \Omega} \int_{\Omega}|K(x-y)| d y<\infty, \quad b:=\sup _{x \in \Omega} \int_{\Omega}|\nabla K(x-y)| d y<\infty .
$$

(V): The viscosity $\nu$ is Lipschitz continuous on $[-1,1]$ and there exists some $\nu_{1}>0$ such that

$$
\nu_{1} \leq \nu(s), \quad \forall s \in[-1,1] .
$$

The mobility $m$ is supposed to be degenerate at \pm 1 , and the potential $F$ is assumed to be singular (e.g., logarithmic like) and defined in $(-1,1)$. More precisely, we assume the condition

(M): The mobility $m$ is Lipschitz continuous on $[-1,1]$, and satisfies $m \geq 0, m(s)=0$ if and only if $s=-1$ or $s=1$. Moreover, there exists some $\epsilon_{0}>0$ such that $m$ is nonincreasing in $\left[1-\epsilon_{0}, 1\right]$ and nondecreasing in $\left[-1,-1+\epsilon_{0}\right]$.

Furthermore, $m$ and $F$ are supposed to fulfill the condition

(A1): $F \in C^{2}(-1,1)$ and $\lambda:=m F^{\prime \prime} \in C[-1,1]$.

Condition (A1) is typical in the analysis of the $\mathrm{CH}$ equation with degenerate mobility (see $[16,31,33,34])$. As far as $F$ is concerned, we assume that it satisfies the following assumptions.

(A2): There exists some $\epsilon_{0}>0$ such that $F^{\prime \prime}$ is nondecreasing in $\left[1-\epsilon_{0}, 1\right)$ and nonincreasing in $\left(-1,-1+\epsilon_{0}\right]$.

(A3): There exists some $c_{0}>0$ such that

$$
F^{\prime \prime}(s) \geq c_{0}, \quad \forall s \in(-1,1) .
$$

(A4): There exists some $\alpha_{0}>0$ such that

$$
m(s) F^{\prime \prime}(s) \geq \alpha_{0}, \quad \forall s \in[-1,1] .
$$

Remark 2.1. It is worth recalling that the assumptions $(\mathbf{M}),(\mathbf{A} \mathbf{1})-(\mathbf{A} \mathbf{4})$ are satisfied, for instance, by (1.8) and (1.9). Also, we recall that any symmetric kernel $K \in W^{1,1}\left(B_{\rho}\right)$, where $B_{\rho}:=\{z \in$ $\left.\mathbb{R}^{d}:|z|<\rho\right\}, \rho:=\operatorname{diam}(\Omega)$, satisfies $(\mathbf{K})$. 
As far as the weak formulation is concerned, we point out that if the mobility degenerates then the gradient of the chemical potential $\mu$ cannot be controlled in some $L^{p}$ space. For this reason, and also in order to pass to the limit to prove the existence of a weak solution, a suitable reformulation of the problem needs to be introduced in such a way that $\mu$ does not appear explicitly (cf. [16], see also [24]). The definition of weak solution given in [24] but modified accordingly to our setting reads as follows.

Definition 2.2. Let $\boldsymbol{u}_{0} \in G_{\text {div }}, \varphi_{0} \in H$ with $F\left(\varphi_{0}\right) \in L^{1}(\Omega), \boldsymbol{v} \in L^{2}\left(0, T ; V_{\text {div }}^{\prime}\right)$, and $0<T<+\infty$ be given. A couple $[\boldsymbol{u}, \varphi]$ is called weak solution to (1.1)-(1.6) on $[0, T]$ if

- $\boldsymbol{u}, \varphi$ satisfy

$$
\begin{aligned}
& \boldsymbol{u} \in L^{\infty}\left(0, T ; G_{\text {div }}\right) \cap L^{2}\left(0, T ; V_{\text {div }}\right), \\
& \boldsymbol{u}_{t} \in L^{4 / 3}\left(0, T ; V_{d i v}^{\prime}\right), \quad \text { if } d=3, \\
& \boldsymbol{u}_{t} \in L^{2}\left(0, T ; V_{d i v}^{\prime}\right), \quad \text { if } d=2, \\
& \varphi \in L^{\infty}(0, T ; H) \cap L^{2}(0, T ; V), \\
& \varphi_{t} \in L^{2}\left(0, T ; V^{\prime}\right),
\end{aligned}
$$

and

$$
\varphi \in L^{\infty}\left(Q_{T}\right), \quad|\varphi(x, t)| \leq 1 \quad \text { for a.e. }(x, t) \in Q_{T}:=\Omega \times(0, T) ;
$$

- for every $\boldsymbol{w} \in V_{\text {div }}$, every $\psi \in V$, and for almost any $t \in(0, T)$ we have that

$$
\begin{aligned}
& \left\langle\boldsymbol{u}_{t}, \boldsymbol{w}\right\rangle_{V_{d i v}}+2(\nu(\varphi) D \boldsymbol{u}, D \boldsymbol{w})+b(\boldsymbol{u}, \boldsymbol{u}, \boldsymbol{w})=((-K * \varphi) \nabla \varphi, \boldsymbol{w})+\langle\boldsymbol{v}, \boldsymbol{w}\rangle, \\
& \left\langle\varphi_{t}, \psi\right\rangle_{V}+\int_{\Omega} m(\varphi) F^{\prime \prime}(\varphi) \nabla \varphi \cdot \nabla \psi d x-\int_{\Omega} m(\varphi) \nabla K * \varphi \cdot \nabla \psi d x=(\boldsymbol{u} \varphi, \nabla \psi) ;
\end{aligned}
$$

- the initial conditions $\boldsymbol{u}(0)=\boldsymbol{u}_{0}, \varphi(0)=\varphi_{0}$, hold true a.e. in $\Omega$.

Observe that the regularity properties of the weak solution imply that

$$
\boldsymbol{u} \in C_{w}\left([0, T] ; G_{d i v}\right), \quad \varphi \in C_{w}([0, T] ; H) .
$$

Therefore, the initial conditions $\boldsymbol{u}(0)=\boldsymbol{u}_{0}, \varphi(0)=\varphi_{0}$, make sense.

The results on existence of weak solutions can be found in [24, Theorem 2]. The uniqueness of a weak solution in the case of constant viscosity is provided in [20, Theorem 4] (cf. Remark 2.6).

Theorem 2.3. Assume that $(\boldsymbol{K}),(\boldsymbol{V}),(\boldsymbol{M})$, and $(\boldsymbol{A} \mathbf{1})-(\boldsymbol{A} 3)$, are satisfied. Let $\boldsymbol{u}_{0} \in G_{\text {div }}$ and $\varphi_{0} \in L^{\infty}(\Omega)$ with $F\left(\varphi_{0}\right) \in L^{1}(\Omega)$ and $M\left(\varphi_{0}\right) \in L^{1}(\Omega)$, where $M \in C^{2}(-1,1)$ is defined by $m(s) M^{\prime \prime}(s)=1$ for all $s \in(-1,1)$ and $M(0)=M^{\prime}(0)=0$. Let also $\boldsymbol{v} \in L_{\text {loc }}^{2}\left([0, \infty) ; V_{\text {div }}^{\prime}\right)$.

(a) Then, for every $T>0$, system (1.1)-(1.6) admits a weak solution $[\boldsymbol{u}, \varphi]$ on $[0, T]$ such that $\bar{\varphi}(t)=\bar{\varphi}_{0}$ for all $t \in[0, T]$. In addition, if $d=2$, then the weak solution $[\boldsymbol{u}, \varphi]$ satisfies the energy equation

$$
\begin{aligned}
& \frac{1}{2} \frac{d}{d t}\left(\|\boldsymbol{u}\|^{2}+\|\varphi\|^{2}\right)+2\|\sqrt{\nu(\varphi)} D \boldsymbol{u}\|^{2}+\int_{\Omega} m(\varphi) F^{\prime \prime}(\varphi)|\nabla \varphi|^{2} d x \\
& =\int_{\Omega} m(\varphi) \nabla K * \varphi \cdot \nabla \varphi d x+\int_{\Omega}(-K * \varphi) \boldsymbol{u} \cdot \nabla \varphi d x+\langle\boldsymbol{v}, \boldsymbol{u}\rangle
\end{aligned}
$$


for almost any $t>0$. In the case $d=3,[\boldsymbol{u}, \varphi]$ satisfies the following energy inequality

$$
\begin{aligned}
& \frac{1}{2}\left(\|\boldsymbol{u}(t)\|^{2}+\|\varphi(t)\|^{2}\right)+2 \int_{0}^{t}\|\sqrt{\nu(\varphi)} D \boldsymbol{u}\|^{2}+\int_{0}^{t} \int_{\Omega} m(\varphi) F^{\prime \prime}(\varphi)|\nabla \varphi|^{2} d x \\
& \leq \frac{1}{2}\left(\left\|\boldsymbol{u}_{0}\right\|^{2}+\left\|\varphi_{0}\right\|^{2}\right)+\int_{0}^{t} \int_{\Omega} m(\varphi) \nabla K * \varphi \cdot \nabla \varphi d x \\
& -\int_{0}^{t} \int_{\Omega} K * \varphi \boldsymbol{u} \cdot \nabla \varphi d x+\int_{0}^{t}\langle\boldsymbol{v}, \boldsymbol{u}\rangle, \quad \forall t>0 .
\end{aligned}
$$

(b) Let $d=2$, and let $\nu$ be constant. In addition, suppose that $(\boldsymbol{A} 4)$ is satisfied. Then the weak solution to system (1.1)-(1.6) is also unique. Moreover, let $\left[\boldsymbol{u}_{i}, \varphi_{i}\right]$ be two weak solutions corresponding to two initial data $\left[\boldsymbol{u}_{0 i}, \varphi_{0 i}\right]$ and external force densities $\boldsymbol{v}_{i}$, with $\boldsymbol{u}_{0 i} \in G_{\text {div }}$, $\varphi_{0 i} \in L^{\infty}(\Omega)$ such that $F\left(\varphi_{0 i}\right) \in L^{1}(\Omega), M\left(\varphi_{0 i}\right) \in L^{1}(\Omega)$ and $\boldsymbol{v}_{i} \in L_{l o c}^{2}\left([0, \infty) ; V_{d i v}^{\prime}\right)$, $i=1,2$. Then, setting $\boldsymbol{u}:=\boldsymbol{u}_{2}-\boldsymbol{u}_{1}, \varphi:=\varphi_{2}-\varphi_{1}$ and $\boldsymbol{v}:=\boldsymbol{v}_{2}-\boldsymbol{v}_{1}$, the following continuous dependence estimate holds true:

$$
\begin{aligned}
& \|\boldsymbol{u}(t)\|^{2}+\|\varphi(t)\|_{V^{\prime}}^{2}+\|\boldsymbol{u}\|_{L^{2}\left(0, t ; V_{\text {div }}\right)}^{2}+\|\varphi\|_{L^{2}(0, t ; H)}^{2} \\
& \leq\left(\|\boldsymbol{u}(0)\|^{2}+\|\varphi(0)\|_{V^{\prime}}^{2}\right) \Lambda_{0}(t)+|\bar{\varphi}(0)|^{2} \Lambda_{1}(t)+\|\boldsymbol{v}\|_{L^{2}\left(0, T ; V_{\text {div }}^{\prime}\right)}^{2} \Lambda_{2}(t),
\end{aligned}
$$

for all $t \in[0, T]$, where $\Lambda_{0}, \Lambda_{1}$ and $\Lambda_{2}$ are continuous functions which depend on the norms of the two solutions. The functions $\Lambda_{i}$ also depend on $F, K, \nu$ and $\Omega$.

Remark 2.4. By a careful look to the argument in [24, Theorem 2] it is easy to see that, as far as the only existence of a weak solution is concerned (cf. point (a) in the statement of Theorem 2.3) the Lipschitz continuity of $\nu$ and $m$ in assumptions (V) and (M) can be weakened into just $\nu, m \in C^{0}([-1,1])$. The condition $m \in C^{0,1}([-1,1])$ is needed for the uniqueness of the weak solution and for the continuous dependence estimate (2.5), while the Lipschitz continuity of both $\nu$ and $m$ will be used in Theorem 3.6.

Remark 2.5. In [24], the viscosity $\nu$ was assumed to be constant just to avoid technicalities, but the results therein also hold for a nonconstant viscosity satisfying $(\mathbf{V})$. Notice that the approximation argument developed in [24] actually requires an upper bound for $\nu$ on the whole of $\mathbb{R}$ (see [24, Proof of Theorem 1]). However, the argument therein can be repeated by working with a (globally) Lipschitz continuous bounded extension $\widetilde{\nu}$ of $\nu$ outside $[-1,1]$ (e.g., we could take $\widetilde{\nu}(s)=\nu( \pm 1)$ for $s \gtrless \pm 1)$. Indeed, in the limit, the weak solution satisfies $|\varphi| \leq 1$ a.e in $\Omega \times(0, T)$.

Remark 2.6. As we already observed in the Introduction, in [24] a slightly different model was considered. All of the results in [24] and, in particular, [24, Theorem 2], can be obviously restated for system (1.1)-(1.4). We also point out that in [24, Theorem 2] an additional condition was assumed. In the present situation, this condition more precisely becomes: there exist some $k>4 a^{*}$ and $\epsilon_{0}>0$ such that

$$
F^{\prime \prime}(s) \geq k, \quad \forall s \in\left(-1,-1+\epsilon_{0}\right] \cup\left[1-\epsilon_{0}, 1\right) .
$$

This assumption, which yields the equicoercivity $F_{\varepsilon}(s) \geq(k / 8) s^{2}-k^{\prime}$, for all $s \in \mathbb{R}$ (with $k^{\prime} \in \mathbb{R}$ independent of $\varepsilon$ ), for the family of $\varepsilon$-regularizations $F_{\varepsilon}$ of $F$, was helpful to deduce the lower bound

$$
\mathcal{E}_{\varepsilon}\left(\boldsymbol{u}_{\varepsilon}, \varphi_{\epsilon}\right)=\frac{1}{2}\left\|\boldsymbol{u}_{\varepsilon}\right\|^{2}-\frac{1}{2}\left(\varphi_{\epsilon}, K * \varphi_{\epsilon}\right)+\int_{\Omega} F_{\varepsilon}\left(\varphi_{\epsilon}\right) \geq \frac{1}{2}\left\|\boldsymbol{u}_{\varepsilon}\right\|^{2}+\frac{1}{2}\left(\frac{k}{4}-a^{*}\right)\left\|\varphi_{\epsilon}\right\|^{2}-k^{\prime \prime}
$$

for the regularized energy associated to the approximate solution $\left[\boldsymbol{u}_{\varepsilon}, \varphi_{\epsilon}\right]$, which, combined with the approximate energy inequality, leads, in turn, to the basic estimates for $\left[\boldsymbol{u}_{\varepsilon}, \varphi_{\epsilon}\right]$. However, 
we now show that (2.6) is superfluous. Indeed, it can be removed by employing a variant of the Elliot-Garcke type of approximation (see [24, Proof of Theorem 2]). More precisely, the following approximation $F_{\varepsilon}$ can be considered (see also [19])

$$
F_{\varepsilon}(s)=\left\{\begin{array}{l}
F(1-\varepsilon)+F^{\prime}(1-\varepsilon)(s-(1-\varepsilon))+\frac{1}{2} F^{\prime \prime}(1-\varepsilon)(s-(1-\varepsilon))^{2} \\
+(s-(1-\varepsilon))^{3}, \quad s \geq 1-\varepsilon, \\
F(s), \quad|s| \leq 1-\varepsilon, \\
F(-1+\varepsilon)+F^{\prime}(-1+\varepsilon)(s-(-1+\varepsilon))+\frac{1}{2} F^{\prime \prime}(-1+\varepsilon)(s-(-1+\varepsilon))^{2} \\
+|s-(-1+\varepsilon)|^{3}, \quad s \leq-1+\varepsilon .
\end{array}\right.
$$

It is easy to check that $F_{\varepsilon} \in C_{l o c}^{2,1}(\mathbb{R})$ and that, thanks also to (A3), there exist two constants $k_{1}>0$ and $k_{2} \in \mathbb{R}$, which do not depend on $\varepsilon$, such that

$$
F_{\varepsilon}(s) \geq k_{1}|s|^{3}-k_{2}, \quad \forall s \in \mathbb{R} .
$$

Moreover, as a consequence of (A3), we still have that

$$
F_{\varepsilon}^{\prime \prime}(s) \geq c_{0}, \quad \forall s \in \mathbb{R}, \quad \text { a.e. } x \in \Omega,
$$

and (A2) implies that there exists $\varepsilon_{0}>0$ such that

$$
F_{\varepsilon}(s) \leq F(s)+\varepsilon^{3}, \quad \forall s \in(-1,1), \quad \forall \varepsilon \in\left(0, \varepsilon_{0}\right] .
$$

Thanks to the bounds (2.7)-(2.9), the argument of [24, Proof of Theorem 2], to which we refer for the details, can still be reproduced, and the same basic estimates for the sequence $\left[\boldsymbol{u}_{\varepsilon}, \varphi_{\varepsilon}\right]$ of approximate solutions can be recovered. Moreover, the argument to prove that $|\varphi| \leq 1$ almost everywhere in $Q_{T}$ is unchanged. There only remains to show that we can still pass to the limit, as $\varepsilon \rightarrow 0$, in the term $\int_{\Omega} m_{\varepsilon}\left(\varphi_{\varepsilon}\right) F_{\varepsilon}^{\prime \prime}\left(\varphi_{\epsilon}\right) \nabla \varphi_{\epsilon} \cdot \nabla \psi$ (for all $\psi \in V$ ), which appears in the variational formulation of the approximate problem, in order to prove that the limit couple $[\boldsymbol{u}, \varphi]$ is a weak solution. To this aim, notice that, due to (A1) and to the convergence $\varphi_{\epsilon} \rightarrow \varphi$, pointwise almost everywhere in $Q_{T}$, it is easy to see that we still have

$$
m_{\varepsilon}\left(\varphi_{\varepsilon}\right) F_{\varepsilon}^{\prime \prime}\left(\varphi_{\epsilon}\right) \rightarrow m(\varphi) F^{\prime \prime}(\varphi), \quad \text { a.e. in } Q_{T} .
$$

Moreover, there holds

$$
\begin{aligned}
\left|m_{\varepsilon}(s) F_{\varepsilon}^{\prime \prime}(s)\right| \leq & \lambda_{\infty}+6 m(1-\varepsilon)(s-(1-\varepsilon)) \chi_{[1-\varepsilon,+\infty)}(s) \\
& +6 m(-1+\varepsilon)|s-(-1+\varepsilon)| \chi_{(-\infty,-1+\varepsilon]}(s),
\end{aligned}
$$

where $\lambda_{\infty}:=\|\lambda\|_{L^{\infty}(-1,1)}$, and $\chi_{E}$ denotes the characteristic function of a set $E \subset \mathbb{R}$. Since $\varphi_{\epsilon}$ is bounded in $L^{r}\left(Q_{T}\right)$, where $r=10 / 3$ if $d=3$, and $r=4$ if $d=2$, then, by Lebesgue's theorem, (2.10) and (2.11) entail that

$$
m_{\varepsilon}\left(\varphi_{\varepsilon}\right) F_{\varepsilon}^{\prime \prime}\left(\varphi_{\epsilon}\right) \rightarrow m(\varphi) F^{\prime \prime}(\varphi), \quad \text { strongly in } L^{r}\left(Q_{T}\right) .
$$

This strong convergence, together with the weak convergence $\varphi_{\varepsilon} \rightarrow \varphi$ in $L^{2}(0, T ; V)$, allows us to pass to the limit in the term above.

Remark 2.7. It is worth pointing out that, to prove the existence of a weak solution (in the sense of Definition 2.2), we do not need that the potential $F$ has some singular behavior at the endpoints $s= \pm 1$ (cf. (A1)-(A3)). Instead, the key role is played by the degenerate mobility, i.e., by condition (M), with $F$ being also $C^{2}(-1,1)$. This is enough to ensure the crucial bound $|\varphi| \leq 1$ almost everywhere in $Q_{T}$. However, concerning uniqueness and regularity results (see the following sections), assumption (A4) is crucial, but it implies that $F$ must have some singular behavior at the endpoints, in the sense that, at least, $F^{\prime \prime}(s) \rightarrow \infty$, as $s \rightarrow \pm 1$. 
Remark 2.8. By combining (A1) with the definition of the function $M$, we can see that $F$ and $M$ are not independent. Actually, in the statement of Theorem 2.3, $F\left(\varphi_{0}\right) \in L^{1}(\Omega)$ is a consequence of $M\left(\varphi_{0}\right) \in L^{1}(\Omega)$. Moreover, if (A4) holds then the two conditions are equivalent (see [24]).

\section{Strong SOlutions in tWo Dimensions}

Here we state and prove our main result: the existence of strong solutions to (1.1)-(1.6). Let us introduce some preliminaries that we shall need in the proof. First of all, we observe that equations (1.2)-(1.3) can formally be rewritten in the form

$$
\varphi_{t}+\boldsymbol{u} \cdot \nabla \varphi=\Delta B(\varphi)-\operatorname{div}(m(\varphi)(\nabla K * \varphi))
$$

where we have set

$$
B(s)=\int_{0}^{s} \lambda(\sigma) d \sigma, \quad \lambda(s)=m(s) F^{\prime \prime}(s), \quad \forall s \in[-1,1] .
$$

Notice that we have $\nabla B(\varphi)=\lambda(\varphi) \nabla \varphi$. Hence, the boundary condition $m(\varphi) \nabla \mu \cdot \boldsymbol{n}=0$ can be rewritten as

$$
[\nabla B(\varphi)-m(\varphi)(\nabla K * \varphi)] \cdot \boldsymbol{n}=0 .
$$

Thus, the equivalent weak formulation of equations (1.2)-(1.3) is

$$
\left\langle\varphi_{t}, \psi\right\rangle_{V}+\int_{\Omega} \nabla B(\varphi) \cdot \nabla \psi d x-\int_{\Omega} m(\varphi)(\nabla K * \varphi) \cdot \nabla \psi d x=(\boldsymbol{u} \varphi, \nabla \psi),
$$

for every $\psi \in V$ and for almost any $t \in(0, T)$.

On account of this formulation, we can give our definition of strong solution if $d=2$.

Definition 3.1. Let $\boldsymbol{u}_{0} \in V_{\text {div }}, \varphi_{0} \in V \cap C^{\beta}(\bar{\Omega})$ for some $\beta \in(0,1), \boldsymbol{v} \in L^{2}\left(0, T ; G_{d i v}\right)$, and $0<T<+\infty$ be given. A weak solution $[\boldsymbol{u}, \varphi]$ to (1.1)-(1.6) on $[0, T]$ corresponding to $\left[\boldsymbol{u}_{0}, \varphi_{0}\right]$ is called strong solution if

$$
\begin{aligned}
& \boldsymbol{u} \in L^{\infty}\left(0, T ; V_{d i v}\right) \cap L^{2}\left(0, T ; H^{2}(\Omega)^{2}\right), \quad \boldsymbol{u}_{t} \in L^{2}\left(0, T ; G_{d i v}\right), \\
& \varphi \in L^{\infty}(0, T ; V) \cap L^{2}\left(0, T ; H^{2}(\Omega)\right), \quad \varphi_{t} \in L^{2}(0, T ; H), \\
& \boldsymbol{u}_{t}-2 \operatorname{div}(\nu(\varphi) D \boldsymbol{u})+(\boldsymbol{u} \cdot \nabla) \boldsymbol{u}+\nabla \widetilde{\pi}=-(K * \varphi) \nabla \varphi+\boldsymbol{v}, \\
& \varphi_{t}+\boldsymbol{u} \cdot \nabla \varphi=\Delta B(\varphi)-\operatorname{div}(m(\varphi)(\nabla K * \varphi)), \\
& \operatorname{div}(\boldsymbol{u})=0
\end{aligned}
$$

almost everywhere in $\Omega \times(0, T)$ with

$$
\boldsymbol{u}=\mathbf{0}, \quad[\nabla B(\varphi)-m(\varphi)(\nabla K * \varphi)] \cdot \boldsymbol{n}=0,
$$

almost everywhere on $\partial \Omega \times(0, T)$, and for some $\tilde{\pi}:=\pi-F(\varphi) \in L^{2}(0, T ; V)$.

Remark 3.2. It is worth noting that, for a strong solution, the nonlocal Cahn-Hilliard equation can also be written in the form

$$
\varphi_{t}+\boldsymbol{u} \cdot \nabla \varphi=\operatorname{div}\left(m(\varphi) F^{\prime \prime}(\varphi) \nabla \varphi-m(\varphi)(\nabla K * \varphi)\right),
$$

almost everywhere in $\Omega \times(0, T)$, while the boundary condition becomes

$$
\left[m(\varphi) F^{\prime \prime}(\varphi) \nabla \varphi-m(\varphi)(\nabla K * \varphi)\right] \cdot \boldsymbol{n}=0,
$$

almost everywhere on $\partial \Omega \times(0, T)$.

Then, we shall use the following lemma to handle the boundary condition (3.3). 
Lemma 3.3. Let $\varphi, \psi \in H^{1 / 2}(\partial \Omega) \cap L^{\infty}(\partial \Omega)$. Then $\varphi \psi \in H^{1 / 2}(\partial \Omega) \cap L^{\infty}(\partial \Omega)$ and

$$
\|\varphi \psi\|_{H^{1 / 2}(\partial \Omega)} \leq\|\varphi\|_{L^{\infty}(\partial \Omega)}\|\psi\|_{H^{1 / 2}(\partial \Omega)}+\|\psi\|_{L^{\infty}(\partial \Omega)}\|\varphi\|_{H^{1 / 2}(\partial \Omega)} .
$$

Proof. The proof is an immediate consequence of the definition of the space $H^{1 / 2}(\partial \Omega)$ with seminorm given by

$$
[\varphi]_{H^{1 / 2}(\partial \Omega)}^{2}=\int_{\partial \Omega} \int_{\partial \Omega} \frac{|\varphi(x)-\varphi(y)|^{2}}{|x-y|^{2}} d \Gamma(x) d \Gamma(y),
$$

where $d \Gamma(\cdot)$ is the surface measure on $\partial \Omega$ (see, e.g., [15, Chapter IX, Section 18]).

To establish the regularity of solutions, we shall also need the kernel $K$ to be more regular than $W_{\text {loc }}^{1,1}$. A possible assumption is that $K \in W_{l o c}^{2,1}\left(\mathbb{R}^{2}\right)$. However, this assumption excludes physically relevant classes of kernels like, e.g., Newtonian and Bessel potential kernels. This class can be included by assuming that $K$ is admissible, according to the following definition.

Definition 3.4. (see $\left[9\right.$, Definition 1]) A kernel $K \in W_{l o c}^{1,1}\left(\mathbb{R}^{d}\right)$ is admissible if the following conditions are satisfied:

(K1): $K \in C^{3}\left(\mathbb{R}^{d} \backslash\{0\}\right)$;

(K2): $K$ is radially symmetric, $K(x)=\tilde{K}(|x|)$ and $\tilde{K}$ is non-increasing;

(K3): $\tilde{K}^{\prime \prime}(r)$ and $\tilde{K}^{\prime}(r) / r$ are monotone on $\left(0, r_{0}\right)$ for some $r_{0}>0$;

(K4): $\left|D^{3} K(x)\right| \leq C_{d}|x|^{-d-1}$ for some $C_{*}>0$.

The advantage of working with admissible kernels is due to the following lemma.

Lemma 3.5. (cf. [9, Lemma 2]) Let $K$ be admissible. Then, for every $p \in(1, \infty)$, there exists $C_{p}>0$ such that

$$
\|\nabla v\|_{L^{p}(\Omega)^{d \times d}} \leq C_{p}\|\psi\|_{L^{p}(\Omega)}, \quad \forall \psi \in L^{p}(\Omega),
$$

where $v=\nabla K * \psi$. Here, $C_{p}=C_{*} p$ for $p \in[2, \infty)$ and $C_{p}=C_{*} p /(p-1)$ for $p \in(1,2)$, for some constant $C_{*}>0$ independent of $p$.

Before stating our result, we need to replace (A1) with the following slightly stronger assumption.

(A1b): $F \in C^{3}(-1,1)$ and $\lambda:=m F^{\prime \prime} \in C^{1}[-1,1]$.

Our main theorem is as follows.

Theorem 3.6. Let the assumptions $(\boldsymbol{K}),(\boldsymbol{V}),(\boldsymbol{M}),(\boldsymbol{A} \mathbf{1 b}),(\boldsymbol{A} 4)$ hold true, and assume that $K \in W_{\text {loc }}^{2,1}\left(\mathbb{R}^{2}\right)$ or that $K$ is admissible $(d=2)$. Let $\boldsymbol{u}_{0} \in G_{\text {div }}, \varphi_{0} \in V \cap L^{\infty}(\Omega)$ with $F\left(\varphi_{0}\right) \in L^{1}(\Omega)$ and $M\left(\varphi_{0}\right) \in L^{1}(\Omega)$, where $M$ is defined as in Theorem 2.3. Let also $\boldsymbol{v} \in L^{2}\left(0, T ; G_{\text {div }}\right)$. Then, for every $T>0$, problem (1.2)-(1.6) admits a weak solution $[\boldsymbol{u}, \varphi]$ on $[0, T]$ such that

$$
\begin{aligned}
& \boldsymbol{u} \in L^{\infty}\left(0, T ; G_{d i v}\right) \cap L^{2}\left(0, T ; V_{d i v}\right), \quad \boldsymbol{u}_{t} \in L^{2}\left(0, T ; V_{d i v}^{\prime}\right), \\
& \varphi \in L^{\infty}(0, T ; V) \cap L^{2}\left(0, T ; H^{2}(\Omega)\right), \quad \varphi_{t} \in L^{2}(0, T ; H) .
\end{aligned}
$$

Assume in addition that $\boldsymbol{u}_{0} \in V_{\text {div }}$ and that $\varphi_{0} \in V \cap C^{\beta}(\bar{\Omega})$ for some $\beta \in(0,1)$. Then problem (1.2)-(1.6) admits a (unique) strong solution satisfying (3.9) and

$$
\boldsymbol{u} \in L^{\infty}\left(0, T ; V_{\text {div }}\right) \cap L^{2}\left(0, T ; H^{2}(\Omega)^{2}\right), \quad \boldsymbol{u}_{t} \in L^{2}\left(0, T ; G_{\text {div }}\right) .
$$

Finally, suppose that $\varphi_{0} \in H^{2}(\Omega)$ and that the following compatibility condition is fulfilled:

$$
\nabla B\left(\varphi_{0}\right) \cdot \boldsymbol{n}=m\left(\varphi_{0}\right)\left(\nabla K * \varphi_{0}\right) \cdot \boldsymbol{n}, \quad \text { a.e. on } \partial \Omega \text {. }
$$

Then the strong solution also satisfies

$$
\varphi \in L^{\infty}\left(0, T ; H^{2}(\Omega)\right), \quad \varphi_{t} \in L^{\infty}(0, T ; H) \cap L^{2}(0, T ; V) .
$$


Remark 3.7. We observe that uniqueness was already proved in [20, Theorem 7]. Actually, a conditional weak-strong uniqueness was established by assuming the existence of a strong solution. That result is no longer a conditional one.

\section{Proof of TheOrem 3.6}

The proof is divided into three main steps. A brief description follows.

Step 1 Assuming that $[\boldsymbol{u}, \varphi]$ is a weak solution in the sense of Definition 2.2, we aim to show that the second component belongs to the regularity class (3.5) provided that $\varphi_{0} \in V \cap L^{\infty}(\Omega)$. We first introduce a regularized problem $P_{\epsilon}$ which is constructed by replacing the singular potential $F$ by a regular one $F_{\epsilon}$, and the degenerate mobility $m$ by a smooth nondegenerate function $m_{\epsilon}>0$. Then we deduce sufficiently strong a priori estimates for $\varphi_{\epsilon}$ that are uniform with respect to $\epsilon>0$. A time discretization scheme applied to the nonlocal Cahn-Hilliard equation with a nondegenerate mobility $m_{\epsilon}$ and regular potential $F_{\epsilon}$ shall be exploited in order to develop a proof-rigorous strategy and all the necessary estimates independent of $\epsilon$.

Step 2 With the improved regularity of $\varphi$ from Step 1, we can then proceed to deduce higher-order estimates for the velocity assuming that $\boldsymbol{u}_{0} \in V_{d i v}$. This will be achieved by means of some crucial arguments that were also exploited in [20] for a similar problem. In this step, the Hölder continuity of $\varphi$ is the main ingredient.

Step 3 We deduce bounds for $\varphi$ in the regularity class (3.12), provided that $\varphi_{0} \in H^{2}(\Omega)$ satisfies some compatibility conditions. This will be carried out by means of the same time discretization scheme as in Step 1, making use of the smoothness of the velocity obtained in Step 2 (see also (3.10)).

Step 1. We first establish the $L^{\infty}(0, T ; V) \cap L^{2}\left(0, T ; H^{2}(\Omega)\right)$ regularity for $\varphi$. For this purpose, we need to carefully deduce higher-order estimates on the nonlocal Cahn-Hilliard equation in such a way that the only regularity which is exploited for $\boldsymbol{u}$ is the weak one, i.e., $\boldsymbol{u} \in$ $L^{\infty}\left(0, T ; G_{\text {div }}\right) \cap L^{2}\left(0, T ; V_{\text {div }}\right)$. Indeed, if the viscosity is nonconstant, we cannot directly apply the classical regularity result [46, Theorem 3.10] for the incompressible Navier-Stokes system in dimension two (which also requires a regularity assumption on the initial velocity $\boldsymbol{u}_{0} \in V_{\text {div }}$ ) and extend the arguments contained in [23].

The (formal) idea is to test $(3.1)$ by $B(\varphi)_{t}=\lambda(\varphi) \varphi_{t}$. In order to make the argument rigorous, let us develop a suitable approximation scheme. We first approximate problem (3.1) and (3.3) with the following one:

$$
\begin{aligned}
& \varphi_{t}+\boldsymbol{u} \cdot \nabla \varphi=\Delta B_{\epsilon}(\varphi)-\operatorname{div}\left(m_{\epsilon}(\varphi)(\nabla K * Q(\varphi))\right), \\
& {\left[\nabla B_{\epsilon}(\varphi)-m_{\epsilon}(\varphi)(\nabla K * Q(\varphi))\right] \cdot \boldsymbol{n}=0,}
\end{aligned}
$$

where we have set

$$
B_{\epsilon}(s)=\int_{0}^{s} \lambda_{\epsilon}(\sigma) d \sigma, \quad \lambda_{\epsilon}(s)=m_{\epsilon}(s) F_{\epsilon}^{\prime \prime}(s), \quad \forall s \in \mathbb{R} .
$$

Here, the singular potential $F$ is replaced by the regular potential $F_{\epsilon}, \epsilon \in(0,1)$, such that

$$
F_{\epsilon}^{\prime \prime}(s)=\left\{\begin{array}{l}
F^{\prime \prime}(1-\epsilon), \quad s \geq 1-\epsilon, \\
F^{\prime \prime}(s), \quad|s| \leq 1-\epsilon, \\
F^{\prime \prime}(-1+\epsilon), \quad s \leq-1+\epsilon,
\end{array}\right.
$$


with $F_{\epsilon}^{(k)}(0)=F^{(k)}(0)$ for $k=0,1$. Moreover, the degenerate mobility $m$ is replaced by

$$
m_{\epsilon}(s)=\left\{\begin{array}{l}
m(1-\epsilon), \quad s \geq 1-\epsilon, \\
m(s), \quad|s| \leq 1-\epsilon, \\
m(-1+\epsilon), \quad s \leq-1+\epsilon .
\end{array}\right.
$$

In the last term of (4.1), $Q: \mathbb{R} \rightarrow \mathbb{R}$ is the truncation function defined as

$$
Q(s)=\max \{-1, \min \{1, s\}\}, \quad \forall s \in \mathbb{R} .
$$

Notice that, thanks to condition (A1), we have the bound $\left|m_{\epsilon}(s) F_{\epsilon}^{\prime \prime}(s)\right| \leq \lambda_{\infty}$, for all $s \in \mathbb{R}$ and for all $\epsilon \in(0,1)$, where $\lambda_{\infty}:=\|\lambda\|_{L^{\infty}(-1,1)}$. Also on account of condition (A4), there holds

$$
0<\alpha_{0} \leq \lambda_{\epsilon}(s)=m_{\epsilon}(s) F_{\epsilon}^{\prime \prime}(s) \leq \lambda_{\infty}, \quad \forall s \in \mathbb{R} .
$$

Moreover, observe that $m_{\epsilon}$ has the following properties:

$$
\begin{aligned}
& 0<\widetilde{m}_{\varepsilon} \leq m_{\epsilon}(s) \leq m_{\infty}, \quad \forall s \in \mathbb{R}, \\
& \left|m_{\epsilon}\left(s_{2}\right)-m_{\epsilon}\left(s_{1}\right)\right| \leq m_{\infty}^{\prime}\left|s_{2}-s_{1}\right|, \quad \forall s_{1}, s_{2} \in \mathbb{R},
\end{aligned}
$$

for some $\varepsilon$-dependent positive constant $\widetilde{m}_{\varepsilon}$, which, for $\varepsilon$ small enough, is given by (cf. (M))

$$
\widetilde{m}_{\varepsilon}=\min \{m(-1+\varepsilon), m(1-\varepsilon)\},
$$

and for some $\varepsilon$-independent constants $m_{\infty}, m_{\infty}^{\prime}$ which, for all $\varepsilon$, are given by $m_{\infty}:=\|m\|_{L^{\infty}(-1,1)}$, $m_{\infty}^{\prime}:=\left\|m^{\prime}\right\|_{L^{\infty}(-1,1)}$. Finally, due to (A1b), we have that

$$
\left|\lambda_{\epsilon}\left(s_{2}\right)-\lambda_{\epsilon}\left(s_{1}\right)\right| \leq \lambda_{\infty}^{\prime}\left|s_{2}-s_{1}\right|, \quad \forall s_{1}, s_{2} \in \mathbb{R},
$$

where $\lambda_{\infty}^{\prime}:=\left\|\lambda^{\prime}\right\|_{L^{\infty}(-1,1)}$ is independent of $\epsilon$.

We now prove that, for every fixed $\epsilon>0$, problem (4.1), (4.2) admits a solution

$$
\varphi=\varphi_{\epsilon} \in L^{\infty}(0, T ; V) \cap L^{2}\left(0, T ; H^{2}(\Omega)\right), \varphi_{t} \in L^{2}(0, T ; H) .
$$

In order to prove this regularity, the choice of the approximation argument is crucial. Indeed, we point out that the use of the Faedo-Galerkin method is problematic. The reason is that testing the projected (4.1) by $\partial_{t} B\left(\varphi_{n}\right)$ (here $\varphi_{n}$ denotes a Faedo-Galerkin approximate solution) is not allowed, since $B\left(\varphi_{n}\right)$ does not belong, in general, to the subspace spanned by the first $n$ elements of the Faedo-Galerkin basis. The problem is the nonconstant mobility. On the other hand, testing by $\partial_{t} \varphi_{n}$ also leads to technical difficulties.

We shall therefore employ a different approximation approach. In particular, the proof will be carried out by means of a time-discretization argument. For simplicity of notation, for the moment we drop the indication of the approximation parameter $\epsilon$. We fix $N \in \mathbb{N}$ and set $\tau=T / N$. We first introduce the following incremental-step problem: for $k=0, \ldots, N-1$, given $\varphi_{k} \in V$, find $\varphi_{k+1} \in V$ that solves

$$
\begin{aligned}
& -\tau \Delta B\left(\varphi_{k+1}\right)+\varphi_{k+1}=\varphi_{k}-\tau \boldsymbol{U}_{k} \cdot \nabla \varphi_{k+1}-\tau \operatorname{div}\left(m\left(\varphi_{k}\right)\left(\nabla K * Q\left(\varphi_{k}\right)\right)\right), \\
& \nabla B\left(\varphi_{k+1}\right) \cdot \boldsymbol{n}=m\left(\varphi_{k}\right)\left(\nabla K * Q\left(\varphi_{k}\right)\right) \cdot \boldsymbol{n}, \quad \text { a.e. on } \partial \Omega .
\end{aligned}
$$

Each $\boldsymbol{U}_{k}$ is given by

$$
\boldsymbol{U}_{k}:=\frac{1}{\tau} \int_{k \tau}^{(k+1) \tau} \boldsymbol{u}(s) d s, \quad k=0, \ldots, N-1
$$

We now claim that (4.9)-(4.10) admits for every $\varphi_{0} \in V$ a solution $\left(\varphi_{1}, \ldots, \varphi_{N}\right) \in H^{2}(\Omega)^{N}$. First, we introduce, for every $k=0, \ldots, N-1$, the nonlinear operator $A_{k}: V \rightarrow V^{\prime}$ defined by

$$
\left\langle A_{k} \varphi, \psi\right\rangle_{V}:=\tau(\nabla B(\varphi), \nabla \psi)+(\varphi, \psi)-\tau\left(\boldsymbol{U}_{k} \varphi, \nabla \psi\right), \quad \forall \varphi, \psi \in V
$$


Then let $g_{k} \in V^{\prime}$ also be given by

$$
\left\langle g_{k}, \psi\right\rangle_{V}:=\left(\varphi_{k}, \psi\right)+\tau\left(m\left(\varphi_{k}\right)\left(\nabla K * Q\left(\varphi_{k}\right)\right), \nabla \psi\right), \quad \forall \psi \in V .
$$

It follows that problem (4.9)-(4.10) can be written as

$$
A_{k} \varphi_{k+1}=g_{k}, \quad \text { in } V^{\prime} .
$$

We now observe that $A_{k}$ is pseudomonotone and coercive on $V$. Indeed, writing the first term on the right-hand side of (4.11) as $\tau(\lambda(\varphi) \nabla \varphi, \nabla \psi)$, it is straightforward to check that $A_{k}$ satisfy all the assumptions of the general results given by [42, Lemma 2.31 and Lemma 2.32] (for pseudomonotonicity) and by [42, Lemma 2.35] (for coercivity). This can be seen by taking $a(x, r, s):=\tau \lambda(r) s-\tau \boldsymbol{U}_{k} r$, $b(x, r):=0$, and $c(x, r, s):=r$ in [42, Lemma 2.31, Lemma 2.32 and Lemma 2.35]. Therefore, (4.12) admits a solution $\varphi_{k+1} \in V$ (see [42, Theorem 2.6]; cf. also [11]).

Using a bootstrap argument, we find that $\varphi_{k+1} \in H^{2}(\Omega)$, for $k=0, \ldots, N-1$. Indeed, owing to (4.6)-(4.7), from (4.9) and (4.10) we deduce that $\Delta B\left(\varphi_{k+1}\right) \in L^{2-\gamma}(\Omega)$, for all $0<\gamma \leq 1$, and $\nabla B\left(\varphi_{k+1}\right) \cdot \boldsymbol{n} \in H^{1 / 2}(\partial \Omega)$. By elliptic regularity theory, we then infer that $B\left(\varphi_{k+1}\right) \in W^{2,2-\gamma}(\Omega)$. Hence, we have $\nabla B\left(\varphi_{k+1}\right) \in W^{1,2-\gamma}(\Omega)^{2}$, for all $0<\gamma \leq 1$. This fact, together with $\nabla B\left(\varphi_{k+1}\right)=$ $\lambda\left(\varphi_{k+1}\right) \nabla \varphi_{k+1}$, implies that $\nabla \varphi_{k+1} \in L^{4}(\Omega)^{2}$. Therefore, the right-hand side of (4.9) belongs to $L^{2}(\Omega)$ and, by applying elliptic regularity theory once again, we deduce that $B\left(\varphi_{k+1}\right) \in H^{2}(\Omega)$. Hence, $\nabla B\left(\varphi_{k+1}\right) \in H^{1}(\Omega)^{2}$ and, therefore thanks to (4.5) and (4.8), it is easy to check that we also have $\nabla \lambda\left(\varphi_{k+1}\right) \in L^{4}(\Omega)^{2}$. Then, again using the fact that $\nabla B\left(\varphi_{k+1}\right)=\lambda\left(\varphi_{k+1}\right) \nabla \varphi_{k+1}$, we deduce that $\nabla \varphi_{k+1} \in H^{1}(\Omega)^{2}$, whence $\varphi_{k+1} \in H^{2}(\Omega)$. Moreover, the following identity, which will be useful later, holds true:

$$
\partial_{i j}^{2} \varphi_{k+1}=\frac{1}{\lambda\left(\varphi_{k+1}\right)} \partial_{i j}^{2} B\left(\varphi_{k+1}\right)-\frac{1}{\lambda^{2}\left(\varphi_{k+1}\right)} \partial_{i} \lambda\left(\varphi_{k+1}\right) \partial_{j} B\left(\varphi_{k+1}\right) .
$$

Let us now begin to establish the basic discrete estimates. We first test (4.9) by $\varphi_{k+1}$ and sum over $k$ from $k=0$ to $k=n$, where $n<N$. By using the following elementary identity

$$
\sum_{k=0}^{n}\left(\varphi_{k+1}-\varphi_{k}, \varphi_{k+1}\right)=\frac{1}{2} \sum_{k=0}^{n}\left\|\varphi_{k+1}-\varphi_{k}\right\|^{2}+\frac{1}{2}\left\|\varphi_{n+1}\right\|^{2}-\frac{1}{2}\left\|\varphi_{0}\right\|^{2},
$$

and the fact that $\nabla B\left(\varphi_{k+1}\right)=\lambda\left(\varphi_{k+1}\right) \nabla \varphi_{k+1}$, we get

$$
\begin{aligned}
& \frac{1}{2} \sum_{k=0}^{n}\left\|\varphi_{k+1}-\varphi_{k}\right\|^{2}+\frac{1}{2}\left\|\varphi_{n+1}\right\|^{2}+\tau \sum_{k=0}^{n}\left(\lambda\left(\varphi_{k+1}\right) \nabla \varphi_{k+1}, \nabla \varphi_{k+1}\right) \\
& =\frac{1}{2}\left\|\varphi_{0}\right\|^{2}+\tau \sum_{k=0}^{n}\left(m\left(\varphi_{k}\right)\left(\nabla K * Q\left(\varphi_{k}\right)\right), \nabla \varphi_{k+1}\right) .
\end{aligned}
$$

Observe that

$$
\tau\left|\sum_{k=0}^{n}\left(m\left(\varphi_{k}\right)\left(\nabla K * Q\left(\varphi_{k}\right)\right), \nabla \varphi_{k+1}\right)\right| \leq \tau \delta \sum_{k=0}^{n}\left\|\nabla \varphi_{k+1}\right\|^{2}+C_{\delta, m, K} T .
$$

Henceforth, we shall denote by $C, C_{\xi}$ some positive constants that may depend on the global data and on the quantities here denoted by $\xi$. The value of $C, C_{\xi}$ may change from line to line or even within the same line. Moreover, $\mathbb{Q} \geq 0$ will stand for a generic monotone nondecreasing continuous function of all its arguments. 
Inserting estimate (4.16) into (4.15), using the lower bound in (4.5), and choosing $\delta>0$ small enough (i.e., $\delta \leq \alpha_{0} / 2$ ), we obtain the discrete inequality

$$
\sum_{k=0}^{n}\left\|\varphi_{k+1}-\varphi_{k}\right\|^{2}+\left\|\varphi_{n+1}\right\|^{2}+\tau \alpha_{0} \sum_{k=0}^{n}\left\|\nabla \varphi_{k+1}\right\|^{2} \leq\left\|\varphi_{0}\right\|^{2}+C_{m, K} T
$$

for $n=0, \ldots, N-1$. The next step now consists in testing (4.9) by $B\left(\varphi_{k+1}\right)-B\left(\varphi_{k}\right)$. We employ the discrete relation (4.14) with $\nabla B\left(\varphi_{k}\right)$ in place of $\varphi_{k}$, the following discrete integration by parts formula

$$
\begin{aligned}
& \tau \sum_{k=0}^{n}\left(m\left(\varphi_{k}\right)\left(\nabla K * Q\left(\varphi_{k}\right)\right), \nabla\left(B\left(\varphi_{k+1}\right)-B\left(\varphi_{k}\right)\right)\right. \\
& =\tau\left(m\left(\varphi_{n+1}\right)\left(\nabla K * Q\left(\varphi_{n+1}\right)\right), \nabla B\left(\varphi_{n+1}\right)\right) \\
& -\tau \sum_{k=0}^{n}\left(m\left(\varphi_{k+1}\right)\left(\nabla K * Q\left(\varphi_{k+1}\right)\right)-m\left(\varphi_{k}\right)\left(\nabla K * Q\left(\varphi_{k}\right)\right), \nabla B\left(\varphi_{k+1}\right)\right) \\
& -\tau\left(m\left(\varphi_{0}\right)\left(\nabla K * Q\left(\varphi_{0}\right)\right), \nabla B\left(\varphi_{0}\right)\right),
\end{aligned}
$$

and the lower bound in (4.5), to get the estimate

$$
\begin{aligned}
& \frac{\alpha_{0}}{\tau} \sum_{k=0}^{n}\left\|\varphi_{k+1}-\varphi_{k}\right\|^{2}+\frac{1}{2}\left\|\nabla B\left(\varphi_{n+1}\right)\right\|^{2}+\frac{1}{2} \sum_{k=0}^{n}\left\|\nabla\left(B\left(\varphi_{k+1}\right)-B\left(\varphi_{k}\right)\right)\right\|^{2} \\
& \leq \frac{1}{2}\left\|\nabla B\left(\varphi_{0}\right)\right\|^{2}+\left(m\left(\varphi_{n+1}\right)\left(\nabla K * Q\left(\varphi_{n+1}\right)\right), \nabla B\left(\varphi_{n+1}\right)\right) \\
& -\sum_{k=0}^{n}\left(m\left(\varphi_{k+1}\right)\left(\nabla K * Q\left(\varphi_{k+1}\right)\right)-m\left(\varphi_{k}\right)\left(\nabla K * Q\left(\varphi_{k}\right)\right), \nabla B\left(\varphi_{k+1}\right)\right) \\
& -\left(m\left(\varphi_{0}\right)\left(\nabla K * Q\left(\varphi_{0}\right)\right), \nabla B\left(\varphi_{0}\right)\right)-\sum_{k=0}^{n}\left(\boldsymbol{U}_{k} \cdot \nabla \varphi_{k+1}, B\left(\varphi_{k+1}\right)-B\left(\varphi_{k}\right)\right) .
\end{aligned}
$$

Let us now estimate individually the terms on the right-hand side of (4.18). We begin with the easier ones. We have

$$
\begin{aligned}
& \left|\left(m\left(\varphi_{n+1}\right)\left(\nabla K * Q\left(\varphi_{n+1}\right)\right), \nabla B\left(\varphi_{n+1}\right)\right)\right| \leq m_{\infty} b|\Omega|^{1 / 2}\left\|\nabla B\left(\varphi_{n+1}\right)\right\| \\
& \leq \frac{1}{4}\left\|\nabla B\left(\varphi_{n+1}\right)\right\|^{2}+C_{m, K, \Omega}, \\
& \left|\sum_{k=0}^{n}\left(m\left(\varphi_{k+1}\right)\left(\nabla K * Q\left(\varphi_{k+1}\right)\right)-m\left(\varphi_{k}\right)\left(\nabla K * Q\left(\varphi_{k}\right)\right), \nabla B\left(\varphi_{k+1}\right)\right)\right| \\
& \leq\left(m_{\infty}^{\prime}|\Omega|^{1 / 2}+m_{\infty}\right) b \sum_{k=0}^{n}\left\|\varphi_{k+1}-\varphi_{k}\right\|\left\|\nabla B\left(\varphi_{k+1}\right)\right\| \\
& \leq \frac{\alpha_{0}}{4 \tau} \sum_{k=0}^{n}\left\|\varphi_{k+1}-\varphi_{k}\right\|^{2}+C_{m, K, \Omega, \alpha_{0}} \tau \sum_{k=0}^{n}\left\|\nabla B\left(\varphi_{k+1}\right)\right\|^{2} .
\end{aligned}
$$


The estimate for the last term on the right-hand side of (4.18) is more delicate. We first observe that, by means of a direct computation, the following bounds can be deduced:

$$
\left\|\boldsymbol{U}_{n}\right\| \leq\|\boldsymbol{u}\|_{L^{\infty}\left(0, T ; G_{d i v}\right)}, \quad \tau \sum_{k=0}^{n}\left\|\nabla \boldsymbol{U}_{k}\right\|^{2} \leq\|\boldsymbol{u}\|_{L^{2}\left(0, T ; V_{d i v}\right)}^{2}, \quad n=0, \ldots, N-1
$$

Then we observe that

$$
\begin{aligned}
\left|\sum_{k=0}^{n}\left(\boldsymbol{U}_{k} \cdot \nabla \varphi_{k+1}, B\left(\varphi_{k+1}\right)-B\left(\varphi_{k}\right)\right)\right| & \leq \frac{\alpha_{0}}{4 \tau} \sum_{k=0}^{n}\left\|\varphi_{k+1}-\varphi_{k}\right\|^{2} \\
& +\frac{\lambda_{\infty}^{2} \tau}{\alpha_{0}} \sum_{k=0}^{n}\left\|\boldsymbol{U}_{k} \cdot \nabla \varphi_{k+1}\right\|^{2} .
\end{aligned}
$$

On the other hand, we have that

$$
\begin{aligned}
& \frac{\lambda_{\infty}^{2} \tau}{\alpha_{0}} \sum_{k=0}^{n}\left\|\boldsymbol{U}_{k} \cdot \nabla \varphi_{k+1}\right\|^{2}=\frac{\lambda_{\infty}^{2} \tau}{\alpha_{0}} \sum_{k=0}^{n}\left\|\boldsymbol{U}_{k} \cdot \frac{1}{\lambda\left(\varphi_{k+1}\right)} \nabla B\left(\varphi_{k+1}\right)\right\|^{2} \\
& \leq \frac{\lambda_{\infty}^{2} \tau}{\alpha_{0}^{3}} \sum_{k=0}^{n}\left\|\boldsymbol{U}_{k}\right\|_{L^{4}(\Omega)^{2}}^{2}\left\|\nabla B\left(\varphi_{k+1}\right)\right\|_{L^{4}(\Omega)^{2}}^{2} \\
& \leq C \tau \sum_{k=0}^{n}\left\|\boldsymbol{U}_{k}\right\|\left\|\nabla \boldsymbol{U}_{k}\right\|\left\|\nabla B\left(\varphi_{k+1}\right)\right\|\left\|B\left(\varphi_{k+1}\right)\right\|_{H^{2}(\Omega)} \\
& \leq \delta \tau \sum_{k=0}^{n}\left\|B\left(\varphi_{k+1}\right)\right\|_{H^{2}(\Omega)}^{2}+C_{\delta} \tau \sum_{k=0}^{n}\left\|\boldsymbol{U}_{k}\right\|^{2}\left\|\nabla \boldsymbol{U}_{k}\right\|^{2}\left\|\nabla B\left(\varphi_{k+1}\right)\right\|^{2} .
\end{aligned}
$$

We proceed to estimate the term in the $H^{2}$-norm of $B\left(\varphi_{k+1}\right)$. By means of a classical elliptic regularity estimate and by $(4.9)$, we find that

$$
\begin{aligned}
& \delta \tau \sum_{k=0}^{n}\left\|B\left(\varphi_{k+1}\right)\right\|_{H^{2}(\Omega)}^{2} \\
& \leq C \delta \tau \sum_{k=0}^{n}\left(\left\|\Delta B\left(\varphi_{k+1}\right)\right\|^{2}+\left\|B\left(\varphi_{k+1}\right)\right\|_{V}^{2}+\left\|\nabla B\left(\varphi_{k+1}\right) \cdot \boldsymbol{n}\right\|_{H^{1 / 2}(\partial \Omega)}^{2}\right) \\
& \leq \frac{C \delta}{\tau} \sum_{k=0}^{n}\left\|\varphi_{k+1}-\varphi_{k}\right\|^{2}+C \delta \tau \sum_{k=0}^{n}\left\|\boldsymbol{U}_{k} \cdot \nabla \varphi_{k+1}\right\|^{2} \\
& +C \delta \tau \sum_{k=0}^{n}\left\|\operatorname{div}\left(m\left(\varphi_{k}\right)\left(\nabla K * Q\left(\varphi_{k}\right)\right)\right)\right\|^{2}+C \delta \tau \sum_{k=0}^{n}\left\|B\left(\varphi_{k+1}\right)\right\|_{V}^{2} \\
& +C \delta \tau \sum_{k=0}^{n}\left\|\nabla B\left(\varphi_{k+1}\right) \cdot \boldsymbol{n}\right\|_{H^{1 / 2}(\partial \Omega)}^{2}
\end{aligned}
$$


As far as the boundary term in (4.24) is concerned, on account of (4.10), we have that

$$
\begin{aligned}
& C \delta \tau \sum_{k=0}^{n}\left\|\nabla B\left(\varphi_{k+1}\right) \cdot \boldsymbol{n}\right\|_{H^{1 / 2}(\partial \Omega)}^{2} \\
& =C \delta \tau \sum_{k=0}^{n}\left\|m\left(\varphi_{k}\right)\left(\nabla K * Q\left(\varphi_{k}\right)\right) \cdot \boldsymbol{n}\right\|_{H^{1 / 2}(\partial \Omega)}^{2} \\
& \leq C \delta \tau \sum_{k=0}^{n}\left(\left\|m\left(\varphi_{k}\right)\right\|_{L^{\infty}(\partial \Omega)}^{2}\left\|\left(\nabla K * Q\left(\varphi_{k}\right)\right) \cdot \boldsymbol{n}\right\|_{H^{1 / 2}(\partial \Omega)}^{2}\right. \\
& \left.+\left\|m\left(\varphi_{k}\right)\right\|_{H^{1 / 2}(\partial \Omega)}^{2}\left\|\left(\nabla K * Q\left(\varphi_{k}\right)\right) \cdot \boldsymbol{n}\right\|_{L^{\infty}(\partial \Omega)}^{2}\right) \\
& \leq C \delta \tau \sum_{k=0}^{n} m_{\infty}^{2}\left\|K * Q\left(\varphi_{k}\right)\right\|_{H^{2}(\Omega)}^{2}+C(\Omega, K) \delta \tau \sum_{k=0}^{n}\left(m_{\infty}^{\prime 2}\left\|\varphi_{k}\right\|_{H^{1 / 2}(\partial \Omega)}^{2}+m_{\infty}^{2}|\partial \Omega|_{1}\right) \\
& \leq C \delta T+C \delta \tau \sum_{k=0}^{n}\left\|\varphi_{k}\right\|_{V}^{2} \\
& \leq C T \delta\left(1+\left\|\varphi_{0}\right\|_{V}^{2}\right) .
\end{aligned}
$$

We break down the main points needed in the series of estimates for (4.25) as follows:

- We have employed Lemma 3.3, the classical trace theorem, and the definition of the space $H^{1 / 2}(\partial \Omega)$, to estimate the term (cf. (3.7))

$$
\left\|m\left(\varphi_{k}\right)\right\|_{H^{1 / 2}(\partial \Omega)}^{2}=\left\|m\left(\varphi_{k}\right)\right\|_{L^{2}(\partial \Omega)}^{2}+\left[m\left(\varphi_{k}\right)\right]_{H^{1 / 2}(\partial \Omega)}^{2}
$$

thanks to (4.7). Moreover, $|\partial \Omega|_{1}$ denotes the one-dimensional measure of $\partial \Omega$.

- Lemma 3.5 is used to estimate the term in the $H^{2}$-norm if $K$ is admissible; otherwise, if $K \in W_{\text {loc }}^{2,1}\left(\mathbb{R}^{d}\right)$, one employs the Young convolution theorem and the fact that $Q$ is bounded (namely, $|Q(s)| \leq 1$ for all $s \in \mathbb{R}$ ) together with the uniform bound (4.17). Clearly, owing to (4.6), $m_{\epsilon}$ is uniformly bounded in $L^{\infty}(\partial \Omega)$-norm.

- The most crucial part of the estimate is the uniform control of the $L^{\infty}(\partial \Omega)-$ norm $^{1}$ of $\partial_{i} K *$ $Q\left(\varphi_{k}\right)$, for each $i=1,2$. We rely on the embedding $W^{1, p}(\Omega) \hookrightarrow C(\bar{\Omega})$, where $p>d=2$ is fixed, and observe that, owing to Lemma 3.5 (or the Young convolution theorem if $K \in$ $W_{\text {loc }}^{2,1}\left(\mathbb{R}^{2}\right)$ ), we have, for each $i=1,2$,

$$
\begin{aligned}
\left\|\partial_{i} K * Q\left(\varphi_{k}\right)\right\|_{L^{\infty}(\partial \Omega)} & \leq C_{p, \Omega}\left\|\partial_{i} K * Q\left(\varphi_{k}\right)\right\|_{W^{1, p}(\Omega)} \\
& \leq C_{p, \Omega, K}\left\|Q\left(\varphi_{k}\right)\right\|_{L^{p}(\Omega)} \\
& \leq C_{p, \Omega, K}
\end{aligned}
$$

since $\left|Q\left(\varphi_{k}\right)\right| \leq 1$ almost everywhere in $\Omega$.

\footnotetext{
${ }^{1}$ Since $\partial \Omega$ is smooth, the normal vector $\boldsymbol{n}=\boldsymbol{n}(x)$ is $C^{1}(\partial \Omega)$.
} 
The third and fourth terms on the right-hand side of (4.24) can be estimated as follows:

$$
\begin{aligned}
C \delta \tau \sum_{k=0}^{n}\left\|\operatorname{div}\left(m\left(\varphi_{k}\right)\left(\nabla K * Q\left(\varphi_{k}\right)\right)\right)\right\|^{2} & \leq C \delta \tau \sum_{k=0}^{n}\left(2 m_{\infty}^{2}\left\|K * Q\left(\varphi_{k}\right)\right\|_{H^{2}(\Omega)}^{2}+2 m_{\infty}^{\prime 2} b^{2}\left\|\nabla \varphi_{k}\right\|^{2}\right) \\
& \leq C_{T} \delta\left(1+\left\|\varphi_{0}\right\|_{V}^{2}\right) \\
C \delta \tau \sum_{k=0}^{n}\left\|B\left(\varphi_{k+1}\right)\right\|_{V}^{2} & \leq C \delta \tau \sum_{k=0}^{n} \lambda_{\infty}^{2}\left\|\varphi_{k+1}\right\|_{V}^{2} \\
& \leq C_{T} \delta\left(1+\left\|\varphi_{0}\right\|^{2}\right)
\end{aligned}
$$

where we have used again Lemma 3.5, (4.6)-(4.7), the basic discrete bound (4.17), and the fact that $\nabla B\left(\varphi_{k+1}\right)=\lambda\left(\varphi_{k+1}\right) \nabla \varphi_{k+1}$.

We now insert (4.25)-(4.28) into (4.24), and then we insert the resulting inequality into (4.23). By fixing $\delta>0$ small enough, we obtain the estimate

$$
\begin{aligned}
\frac{\lambda_{\infty}^{2} \tau}{\alpha_{0}} \sum_{k=0}^{n}\left\|\boldsymbol{U}_{k} \cdot \nabla \varphi_{k+1}\right\|^{2} & \leq \frac{\alpha_{0}}{4 \tau} \sum_{k=0}^{n}\left\|\varphi_{k+1}-\varphi_{k}\right\|^{2} \\
& +C \tau \sum_{k=0}^{n}\left\|\boldsymbol{U}_{k}\right\|^{2}\left\|\nabla \boldsymbol{U}_{k}\right\|^{2}\left\|\nabla B\left(\varphi_{k+1}\right)\right\|^{2}+C_{T}\left(1+\left\|\varphi_{0}\right\|_{V}^{2}\right) .
\end{aligned}
$$

By employing (4.29), (4.19)-(4.20) and (4.22), from (4.18) we get

$$
\begin{aligned}
& \frac{1}{\tau} \sum_{k=0}^{n}\left\|\varphi_{k+1}-\varphi_{k}\right\|^{2}+\left\|\nabla B\left(\varphi_{n+1}\right)\right\|^{2}+\sum_{k=0}^{n}\left\|\nabla\left(B\left(\varphi_{k+1}\right)-B\left(\varphi_{k}\right)\right)\right\|^{2} \\
& \leq C_{T}\left(1+\left\|\varphi_{0}\right\|_{V}^{2}\right)+C \sum_{k=0}^{n}\left(\tau+\tau\left\|\boldsymbol{U}_{k}\right\|^{2}\left\|\nabla \boldsymbol{U}_{k}\right\|^{2}\right)\left\|\nabla B\left(\varphi_{k+1}\right)\right\|^{2} \\
& \leq C_{T}\left(1+\left\|\varphi_{0}\right\|_{V}^{2}\right)+C\left(\tau+\|\boldsymbol{u}\|_{L^{\infty}\left(0, T ; G_{d i v}\right)}^{2} \tau\left\|\nabla \boldsymbol{U}_{n}\right\|^{2}\right)\left\|\nabla B\left(\varphi_{n+1}\right)\right\|^{2} \\
& +C \sum_{k=0}^{n-1}\left(\tau+\tau\left\|\boldsymbol{U}_{k}\right\|^{2}\left\|\nabla \boldsymbol{U}_{k}\right\|^{2}\right)\left\|\nabla B\left(\varphi_{k+1}\right)\right\|^{2} .
\end{aligned}
$$

Observe now that

$$
\tau\left\|\nabla \boldsymbol{U}_{n}\right\|^{2} \leq \int_{n \tau}^{(n+1) \tau}\|\nabla \boldsymbol{u}(s)\|^{2} d s .
$$

Hence, for every $\eta>0$, there exists some $\tau_{\eta}>0$, which only depends on $\eta$ (and on $\boldsymbol{u}$ ), such that $\tau\left\|\nabla \boldsymbol{U}_{n}\right\|^{2}<\eta$ for all $0<\tau<\tau_{\eta}$ and for all $n<N$. By using this fact, we can take $\tau$ small enough in such a way that the third term on the right-hand side of the foregoing inequality (4.30) can be absorbed into the term $\left\|\nabla B\left(\varphi_{n+1}\right)\right\|^{2}$ on the left-hand side. Therefore, on account of (4.21), by applying the discrete Gronwall's Lemma to the ensuing discrete inequality, we obtain from (4.30) that

$$
\begin{aligned}
& \frac{1}{\tau} \sum_{k=0}^{n}\left\|\varphi_{k+1}-\varphi_{k}\right\|^{2}+\left\|\nabla B\left(\varphi_{n+1}\right)\right\|^{2}+\sum_{k=0}^{n}\left\|\nabla\left(B\left(\varphi_{k+1}\right)-B\left(\varphi_{k}\right)\right)\right\|^{2} \\
& \leq \mathbb{Q}\left(\left\|\varphi_{0}\right\|_{V},\|\boldsymbol{u}\|_{L^{\infty}\left(0, T ; G_{d i v}\right) \cap L^{2}\left(0, T ; V_{d i v}\right)}\right), \quad n=0, \ldots, N-1 .
\end{aligned}
$$


We can now proceed to prove the $L^{2}\left(0, T ; H^{2}(\Omega)\right)$-regularity for $\varphi$. Let us first notice that (4.24), combined with (4.25)-(4.29), (4.21) and (4.31), implies that

$$
\tau \sum_{k=0}^{n}\left\|B\left(\varphi_{k+1}\right)\right\|_{H^{2}(\Omega)}^{2} \leq \mathbb{Q}\left(\left\|\varphi_{0}\right\|_{V},\|\boldsymbol{u}\|_{L^{\infty}\left(0, T ; G_{d i v}\right) \cap L^{2}\left(0, T ; V_{d i v}\right)}\right), \quad n=0, \ldots, N-1 .
$$

This estimate yields, in particular, a control on the gradient of $B\left(\varphi_{k+1}\right)$ in $L^{p}(\Omega)$, for $2<p<\infty$. Indeed, from (4.32) we have

$$
\tau \sum_{k=0}^{n}\left\|\nabla B\left(\varphi_{k+1}\right)\right\|_{V}^{2} \leq \mathbb{Q}\left(\left\|\varphi_{0}\right\|_{V},\|\boldsymbol{u}\|_{L^{\infty}\left(0, T ; G_{d i v}\right) \cap L^{2}\left(0, T ; V_{d i v}\right)}\right) .
$$

This bound, together with (2.1) and (4.31), implies that

$$
\tau \sum_{k=0}^{n}\left\|\nabla B\left(\varphi_{k+1}\right)\right\|_{L^{p}(\Omega)}^{2 p /(p-2)} \leq \mathbb{Q}\left(\left\|\varphi_{0}\right\|_{V},\|\boldsymbol{u}\|_{L^{\infty}\left(0, T ; G_{d i v}\right) \cap L^{2}\left(0, T ; V_{d i v}\right)}\right) .
$$

Clearly, thanks to $\nabla B\left(\varphi_{k+1}\right)=\lambda\left(\varphi_{k+1}\right) \nabla \varphi_{k+1}$ and to the bound

$$
\left\|\nabla \lambda\left(\varphi_{k+1}\right)\right\|_{L^{p}(\Omega)^{2}} \leq \lambda_{\infty}^{\prime}\left\|\nabla \varphi_{k+1}\right\|_{L^{p}(\Omega)^{2}},
$$

we can also conclude from (4.33) that

$$
\begin{aligned}
& \tau \sum_{k=0}^{n}\left\|\nabla \varphi_{k+1}\right\|_{L^{p}(\Omega)^{2}}^{2 p /(p-2)}+\tau \sum_{k=0}^{n}\left\|\nabla \lambda\left(\varphi_{k+1}\right)\right\|_{L^{p}(\Omega)^{2}}^{2 p /(p-2)} \\
& \leq \mathbb{Q}\left(\left\|\varphi_{0}\right\|_{V},\|\boldsymbol{u}\|_{L^{\infty}\left(0, T ; G_{d i v}\right) \cap L^{2}\left(0, T ; V_{d i v}\right)}\right) .
\end{aligned}
$$

Thus, using (4.33), (4.34) (written for $p=4$ ), and (4.32), we can infer from (4.13) the desired bound

$$
\tau \sum_{k=0}^{n}\left\|\varphi_{k+1}\right\|_{H^{2}(\Omega)}^{2} \leq \mathbb{Q}\left(\left\|\varphi_{0}\right\|_{V},\|\boldsymbol{u}\|_{L^{\infty}\left(0, T ; G_{d i v}\right) \cap L^{2}\left(0, T ; V_{d i v}\right)}\right), \quad n=0, \ldots, N-1 .
$$

We now need to introduce the functions $\widehat{\varphi}_{N}, \bar{\varphi}_{N}$, and $\widetilde{\varphi}_{N}$ which interpolate the values $\varphi_{n}$ piecewise linearly, backward, and forward constantly, respectively, on the partition. Namely, we set

$$
\left\{\begin{array}{l}
\widehat{\varphi}_{N}(t):=\gamma_{n}(t) \varphi_{n}+\left(1-\gamma_{n}(t)\right) \varphi_{n+1}, \quad \gamma_{n}(t):=n+1-(t / \tau) \\
\bar{\varphi}_{N}(t):=\varphi_{n+1} \\
\widetilde{\varphi}_{N}(t):=\varphi_{n}
\end{array}\right.
$$

for $n \tau<t<(n+1) \tau, n=0, \ldots, N-1$. As a consequence of the estimates (4.17), (4.31) and (4.35), we find the estimate

$$
\begin{aligned}
& \left\|\widehat{\varphi}_{N}^{\prime}\right\|_{L^{2}(0, T ; H)}^{2}+\left\|\widehat{\varphi}_{N}\right\|_{L^{\infty}(0, T ; V)}^{2}+\left\|\bar{\varphi}_{N}\right\|_{L^{\infty}(0, T ; V)}^{2}+\left\|\widetilde{\varphi}_{N}\right\|_{L^{\infty}(0, T ; V)}^{2}+\left\|\bar{\varphi}_{N}\right\|_{L^{2}\left(0, T ; H^{2}(\Omega)\right)}^{2} \\
& +\frac{3}{\tau}\left\|\widehat{\varphi}_{N}-\bar{\varphi}_{N}\right\|_{L^{2}(0, T ; H)}^{2}+\frac{3}{\tau}\left\|\widehat{\varphi}_{N}-\widetilde{\varphi}_{N}\right\|_{L^{2}(0, T ; H)}^{2} \\
& \leq \mathbb{Q}\left(\left\|\varphi_{0}\right\|_{V},\|\boldsymbol{u}\|_{L^{\infty}\left(0, T ; G_{d i v}\right) \cap L^{2}\left(0, T ; V_{d i v}\right)}\right) .
\end{aligned}
$$

Moreover, (4.17), (4.31) and (4.32) also yield that

$$
\left\|B\left(\bar{\varphi}_{N}\right)\right\|_{L^{\infty}(0, T ; V)}+\left\|B\left(\bar{\varphi}_{N}\right)\right\|_{L^{2}\left(0, T ; H^{2}(\Omega)\right)} \leq \mathbb{Q}\left(\left\|\varphi_{0}\right\|_{V},\|\boldsymbol{u}\|_{L^{\infty}\left(0, T ; G_{d i v}\right) \cap L^{2}\left(0, T ; V_{\text {div }}\right)}\right) .
$$

Problem (4.9)-(4.10) can be rewritten in terms of the interpolating functions $\widehat{\varphi}_{N}, \bar{\varphi}_{N}, \widetilde{\varphi}_{N}$ as follows:

$$
\begin{aligned}
& \widehat{\varphi}_{N}^{\prime}=\Delta B\left(\bar{\varphi}_{N}\right)+\boldsymbol{u}_{N} \cdot \nabla \bar{\varphi}_{N}-\operatorname{div}\left(m\left(\widetilde{\varphi}_{N}\right)\left(\nabla K * Q\left(\widetilde{\varphi}_{N}\right)\right)\right), \\
& \nabla B\left(\bar{\varphi}_{N}\right) \cdot \boldsymbol{n}=m\left(\widetilde{\varphi}_{N}\right)\left(\nabla K * Q\left(\widetilde{\varphi}_{N}\right)\right) \cdot \boldsymbol{n} \quad \text { a.e. on } \partial \Omega .
\end{aligned}
$$


Here, each $\boldsymbol{u}_{N}$ is defined by $\boldsymbol{u}_{N}(t):=\boldsymbol{U}_{n}$, for $n \tau<t<(n+1) \tau, n=0, \ldots, N-1$. The variational formulation for (4.38)-(4.39) then reads, for all $\psi \in V$,

$$
\left\langle\widehat{\varphi}_{N}^{\prime}, \psi\right\rangle_{V}+\left(\nabla B\left(\bar{\varphi}_{N}\right), \nabla \psi\right)=-\left(\boldsymbol{u}_{N} \bar{\varphi}_{N}, \nabla \psi\right)+\left(m\left(\widetilde{\varphi}_{N}\right)\left(\nabla K * Q\left(\widetilde{\varphi}_{N}\right)\right), \nabla \psi\right) .
$$

Owing to (4.36), and employing classical compactness results, we deduce that there exists $\varphi \in$ $L^{\infty}(0, T ; V) \cap L^{2}\left(0, T ; H^{2}(\Omega)\right)$ with $\varphi_{t} \in L^{2}(0, T ; H)$, such that, at least for a subsequence, we have

$$
\begin{aligned}
& \widehat{\varphi}_{N} \rightarrow \varphi, \quad \text { weakly* in } L^{\infty}(0, T ; V), \\
& \widehat{\varphi}_{N}^{\prime} \rightarrow \varphi_{t}, \quad \text { weakly in } L^{2}(0, T ; H), \\
& \widehat{\varphi}_{N} \rightarrow \varphi, \quad \text { strongly in } C^{0}\left([0, T] ; L^{q}(\Omega)\right), \quad 2 \leq q<\infty, \\
& \bar{\varphi}_{N} \rightarrow \varphi, \quad \text { weakly* in } L^{\infty}(0, T ; V), \quad \text { weakly in } L^{2}\left(0, T ; H^{2}(\Omega)\right), \\
& \widetilde{\varphi}_{N} \rightarrow \varphi, \quad \text { weakly* in } L^{\infty}(0, T ; V), \\
& \bar{\varphi}_{N} \rightarrow \varphi, \quad \text { strongly in } L^{2}(0, T ; H), \\
& \widetilde{\varphi}_{N} \rightarrow \varphi, \quad \text { strongly in } L^{2}(0, T ; H), \\
& B\left(\bar{\varphi}_{N}\right) \rightarrow B(\varphi), \quad \text { weakly* in } L^{\infty}(0, T ; V), \quad \text { weakly in } L^{2}\left(0, T ; H^{2}(\Omega)\right) .
\end{aligned}
$$

Since $\widetilde{\varphi}_{N} \rightarrow \varphi$ pointwise almost everywhere in $\Omega \times(0, T)$, by virtue of the boundedness of the functions $m, Q$, and by Lebesgue's theorem, we also have that

$$
m\left(\widetilde{\varphi}_{N}\right) \rightarrow m(\varphi), \quad Q\left(\widetilde{\varphi}_{N}\right) \rightarrow Q(\varphi), \quad \text { strongly in } L^{q}(\Omega), \quad 2 \leq q<\infty .
$$

Moreover,

$$
\boldsymbol{u}_{N} \rightarrow \boldsymbol{u}, \quad \text { strongly in } L^{2}\left(0, T ; V_{d i v}\right) .
$$

Indeed, it easy to check that $\boldsymbol{u}_{N}=P_{N} \boldsymbol{u}$, where $P_{N}$ is the projector in $L^{2}\left(V_{\text {div }}\right)$ onto the subspace $\mathcal{S}_{N}:=\left\{\boldsymbol{v} \in L^{2}\left(0, T ; V_{\text {div }}\right):\left.\boldsymbol{v}\right|_{(n \tau,(n+1) \tau)}=\boldsymbol{v}_{n}, \boldsymbol{v}_{n} \in V_{\text {div }}, n=0, \ldots, N-1\right\}$. Since $\cup_{N \geq 1} \mathcal{S}_{N}$ is dense in $L^{2}\left(V_{\text {div }}\right)$, then (4.50) follows.

By means of the weak and strong convergences (4.41)-(4.50), we can now pass to the limit in (4.40) in a standard fashion, and recover the weak formulation of problem (4.1)-(4.2). Notice that we can also pass to the limit directly in (4.38)-(4.39) and prove that (4.1)-(4.2) are satisfied also strongly almost everywhere in $\Omega \times(0, T)$ and on $\partial \Omega \times(0, T)$, respectively.

We thus have shown that, for every $\epsilon>0$, problem (4.1)-(4.2) admits a solution

$$
\varphi_{\epsilon} \in H^{1}(0, T ; H) \cap L^{\infty}(0, T ; V) \cap L^{2}\left(0, T ; H^{2}(\Omega)\right) .
$$

We can also see, by passing to the liminf in (4.36), that the sequence of $\varphi_{\epsilon}$ is uniformly bounded with respect to $\epsilon$ in these spaces (just recall that all constants in (4.5)-(4.8) are independent of $\epsilon$ ). Therefore, there exists a limit function, which we still denote by

$$
\varphi \in H^{1}(0, T ; H) \cap L^{\infty}(0, T ; V) \cap L^{2}\left(0, T ; H^{2}(\Omega)\right),
$$

such that, at least for a subsequence, the same convergences as (4.41)-(4.49) hold for the sequence of $\varphi_{\epsilon}$ to $\varphi$. These convergences allow us to pass to the limit in the variational formulation of problem (4.1)-(4.2) and to recover the variational formulation of the following problem:

$$
\begin{aligned}
& \varphi_{t}+\boldsymbol{u} \cdot \nabla \varphi=\Delta B(\varphi)-\operatorname{div}(m(\varphi)(\nabla K * Q(\varphi))), \\
& {[\nabla B(\varphi)-m(\varphi)(\nabla K * Q(\varphi))] \cdot \boldsymbol{n}=0, \quad \text { on } \partial \Omega \times(0, T) .}
\end{aligned}
$$

We now show that $\varphi$ satisfies the bound $|\varphi| \leq 1$, almost everywhere in $\Omega \times(0, T)$. This allows us to remove the function $Q$ in problem (4.51)-(4.52) and to conclude that indeed the limit solution 
$\varphi$ solves problem (3.1) and (3.3). To this end, we recall that we have just established that $\varphi=\varphi_{\epsilon}$ also satisfies the weak formulation (cf. Definition 2.2) associated with the problem

$$
\begin{aligned}
& \varphi_{t}+\boldsymbol{u} \cdot \nabla \varphi=\operatorname{div}\left(m_{\epsilon}(\varphi) \nabla \mu\right), \\
& \mu=-K * Q(\varphi)+F_{\epsilon}^{\prime}(\varphi), \\
& m_{\epsilon}(\varphi) \nabla \mu \cdot \boldsymbol{n}=0, \quad \text { on } \partial \Omega \times(0, T) .
\end{aligned}
$$

We can therefore argue as in [24, Proof of Theorem 2]. More precisely, we introduce the $C^{2}$ function $M_{\epsilon}$ defined by $m_{\epsilon}(s) M_{\epsilon}^{\prime \prime}(s)=1$, for all $s \in \mathbb{R}, M_{\epsilon}(0)=M_{\epsilon}^{\prime}(0)=0$, and we test (4.53) by $M_{\epsilon}^{\prime}\left(\varphi_{\epsilon}\right)$. This gives the estimate

$$
\frac{d}{d t} \int_{\Omega} M_{\epsilon}\left(\varphi_{\epsilon}\right)+\frac{c_{0}}{2}\left\|\nabla \varphi_{\epsilon}\right\|^{2} \leq \mathbb{Q}\left(\left\|\varphi_{0}\right\|_{V},\|\boldsymbol{u}\|_{L^{\infty}\left(0, T ; G_{d i v}\right) \cap L^{2}\left(0, T ; V_{\text {div }}\right)}\right),
$$

where $c_{0}=\alpha_{0} / m_{\infty}$. Thus, on account of the fact that for $\epsilon$ small enough we have $M_{\epsilon}(s) \leq M(s)$ for all $s \in(-1,1)$ (cf. (M)), and recalling that $M\left(\varphi_{0}\right) \in L^{1}(\Omega)$, we deduce the bound

$$
\left\|M_{\epsilon}\left(\varphi_{\epsilon}\right)\right\|_{L^{\infty}\left(0, T ; L^{1}(\Omega)\right)} \leq \mathbb{Q}\left(\left\|\varphi_{0}\right\|_{V},\|\boldsymbol{u}\|_{L^{\infty}\left(0, T ; G_{d i v}\right) \cap L^{2}\left(0, T ; V_{\text {div }}\right)}\right) .
$$

We can now follow the same lines of [24, Proof of Theorem 2], which rely on an argument devised in [16, Proof of Theorem 1] (see also [12, Proof of Theorem 2.3]), to deduce the desired claim. This concludes the proof of the first part of the theorem. Namely, there exists a weak solution such that $\varphi$ is a bit smoother in the regularity class of (3.9). This concludes Step 1.

Step 2. We now establish the $L^{\infty}\left(0, T ; V_{\text {div }}\right) \cap L^{2}\left(0, T ; H^{2}(\Omega)^{2}\right)$-regularity for $\boldsymbol{u}$, assuming that $\boldsymbol{u}_{0} \in V_{\text {div }}$ and $\varphi_{0} \in V \cap C^{\beta}(\bar{\Omega})$ for some $\beta \in(0,1)$. The argument, which (formally) consists in testing the Navier-Stokes equations (1.1) by $\boldsymbol{u}_{t}$, follows exactly the lines of [20, Proof of Theorem 5 , Step 2]. The key tool is a regularity result for the inhomogeneous Stokes system in non-divergence form, namely,

$$
\begin{cases}-\omega(x) \Delta \boldsymbol{u}+\nabla \pi=\boldsymbol{f}(x), & \text { in } \Omega, \\ \operatorname{div}(\boldsymbol{u})=0, & \text { in } \Omega, \\ u=0, & \text { on } \partial \Omega,\end{cases}
$$

taken from [45]. We report this result here, for the reader's convenience (see also [4] as an alternative route to the $H^{2}$-regularity in the case of a variable viscosity in the Stokes equations).

Proposition 4.1. [45, Proposition 2.1] Let $\boldsymbol{f} \in L^{2}(\Omega)^{2}$ and $\omega \in C^{\delta}(\bar{\Omega})$, for some $\delta \in(0,1)$, such that $0<\lambda_{0} \leq \omega(x) \leq \lambda_{1}<\infty$ for all $x \in \bar{\Omega}$. Then any solution $[\boldsymbol{u}, \pi] \in H^{2}(\Omega)^{2} \times H^{1}(\Omega)$ of $(4.56)$ satisfies the estimate

$$
\|\boldsymbol{u}\|_{H^{2}(\Omega)^{2}}+\|\pi\|_{H^{1}(\Omega)} \leq C\left(\|\boldsymbol{f}\|_{L^{2}(\Omega)^{2}}+\|\pi\|_{L^{2}(\Omega)}\right)
$$

for some constant $C=C\left(\lambda_{0}, \lambda_{1}, \Omega,\|\omega\|_{C^{\delta}(\bar{\Omega})}\right)>0$.

This result is applied to the Navier-Stokes system (1.1) after writing it in the form

$$
-\nu(\varphi) \Delta \boldsymbol{u}+\nabla \hat{\pi}=\boldsymbol{f}
$$

where

$$
\boldsymbol{f}:=(-K * \varphi) \nabla \varphi+\boldsymbol{v}-(\boldsymbol{u} \cdot \nabla) \boldsymbol{u}-\boldsymbol{u}_{t}+2 \nu^{\prime}(\varphi) D \boldsymbol{u} \nabla \varphi, \quad \hat{\pi}:=\pi-F(\varphi) .
$$

This procedure effectively allows to bound the $H^{2}$-norm of $\boldsymbol{u}$ as a function of the $L^{2}$-norm of $\boldsymbol{u}_{t}$. The only remaining ingredient is the Hölder regularity for $\varphi$ (this, in turn, implies Hölder regularity for $\nu(\varphi)$, which is required in order to apply Proposition 4.1). We therefore need to suitably extend the argument of [20, Lemma 2], where the Hölder regularity for a bounded weak solution to 
the convective nonlocal Cahn-Hilliard equation with constant mobility and regular potential was proved. This can be done thanks to assumptions (A1) and (A4). More precisely, we can prove the following result.

Lemma 4.2. Let $d=2$, and assume (A1) and (A4). Let $\boldsymbol{u} \in L^{\infty}\left(T^{\prime}, T ; G_{\text {div }}\right) \cap L^{2}\left(T^{\prime}, T ; V_{\text {div }}\right)$, for some $T>T^{\prime} \geq 0$ and let $\varphi$ be a bounded weak solution to (1.2), (1.3), (1.5)2. Then there exist constants $C>0$ and $\alpha \in(0,1)$ depending on $\|\varphi\|_{L^{\infty}\left(Q_{T^{\prime}, T}\right)}$ and on $\|\boldsymbol{u}\|_{L^{4}\left(Q_{T^{\prime}, T}\right)}$, respectively, such that

$$
|\varphi(x, t)-\varphi(y, s)| \leq C\left(|x-y|^{\alpha}+|t-s|^{\alpha / 2}\right),
$$

for every $(x, t),(y, s) \in \bar{Q}_{T^{\prime}, T}:=\bar{\Omega} \times\left[T^{\prime}, T\right]$.

Proof. Following the lines of [20, Proof of Lemma 2] (cf. also [40]), let $k \in \mathbb{R}$ and $\eta=\eta(x, t) \in$ $[0,1]$ be a continuous piecewise-smooth function which is supported on the space-time cylinders $Q_{t_{0}, t_{0}+\tau}(r):=B_{r}\left(x_{0}\right) \times\left(t_{0}, t_{0}+\tau\right)$, where $B_{r}\left(x_{0}\right)$ denotes the (open) ball centered at $x_{0}$ of radius $r>0$. As usual for the interior Hölder regularity, one takes $x_{0} \in \Omega$, while $x_{0} \in \partial \Omega$ for the corresponding boundary estimate, and then exploits a standard compactness argument, in which $\bar{\Omega}$ may be covered by a finite number of such balls. We thus multiply (1.2), (1.3), which can be written as

$$
\varphi_{t}+\boldsymbol{u} \cdot \nabla \varphi=\operatorname{div}(\lambda(\varphi) \nabla \varphi+\boldsymbol{\kappa}), \quad \boldsymbol{\kappa}(x, t):=-m(\varphi)(\nabla K * \varphi),
$$

by $\eta^{2} \varphi_{k}^{+}$, where $\varphi_{k}^{+}:=\max \{0, \varphi-k\}$, integrate the resulting identity over $Q_{t_{0}, t}:=\Omega \times\left(t_{0}, t\right)$, where $T^{\prime} \leq t_{0}<t<t_{0}+\tau \leq T$, to deduce that

$$
\begin{aligned}
& \int_{Q_{t_{0}, t}} \varphi_{t} \eta^{2} \varphi_{k}^{+} d x d t+\int_{Q_{t_{0}, t}} \lambda(\varphi) \nabla \varphi_{k}^{+} \cdot \nabla\left(\eta^{2} \varphi_{k}^{+}\right) d x d t \\
& =\int_{Q_{t_{0}, t}} \boldsymbol{u} \varphi \cdot \nabla\left(\eta^{2} \varphi_{k}^{+}\right) d x d t+\int_{Q_{t_{0}, t}} \boldsymbol{\kappa}(x, t) \cdot \nabla\left(\eta^{2} \varphi_{k}^{+}\right) d x d t .
\end{aligned}
$$

Since we have $\nabla \varphi_{k}^{+} \cdot \nabla\left(\eta^{2} \varphi_{k}^{+}\right)=\left|\nabla\left(\eta \varphi_{k}^{+}\right)\right|^{2}-|\nabla \eta|^{2}\left(\varphi_{k}^{+}\right)^{2}$, we obtain from (4.59) and the assumption (A4) that

$$
\begin{aligned}
\frac{1}{2} \sup _{s \in\left(t_{0}, t\right)} & \int_{\Omega}\left(\eta \varphi_{k}^{+}\right)^{2}(s) d x+\alpha_{0} \int_{Q_{t_{0}, t}}\left|\nabla\left(\eta \varphi_{k}^{+}\right)\right|^{2} d x d t \\
& \leq \frac{1}{2} \int_{\Omega}\left(\eta \varphi_{k}^{+}\right)^{2}\left(t_{0}\right) d x+\int_{Q_{t_{0}, t}}\left(\varphi_{k}^{+}\right)^{2}\left|\eta \eta_{t}\right| d x d t \\
& +\lambda_{\infty} \int_{Q_{t_{0}, t}}\left(\varphi_{k}^{+}\right)^{2}|\nabla \eta|^{2} d x d t+\int_{Q_{t_{0}, t}} \boldsymbol{u} \varphi \cdot \nabla\left(\eta^{2} \varphi_{k}^{+}\right) d x d t \\
& +\int_{Q_{t_{0}, t}} \boldsymbol{\kappa}(x, t) \cdot \nabla\left(\eta^{2} \varphi_{k}^{+}\right) d x d t .
\end{aligned}
$$

The fourth term on the right-hand side of (4.60) can still be estimated in the same fashion as in [45, Proof of Lemma 3.2], using the fact that $\boldsymbol{u} \in L^{4}\left(Q_{T^{\prime}, T}\right)$ is divergence free. Arguing by the elementary Hölder's and Young's inequalities, we find that

$$
\begin{aligned}
& \left|\int_{Q_{t_{0}, t}} \boldsymbol{u} \varphi \cdot \nabla\left(\eta^{2} \varphi_{k}^{+}\right) d x d t\right| \\
& \leq \frac{1}{4}\left\|\eta \varphi_{k}^{+}\right\|_{L^{\infty}\left(t_{0}, t ; H\right)}^{2}+\frac{1}{4} \alpha_{0}\left\|\nabla\left(\eta \varphi_{k}^{+}\right)\right\|_{L^{2}\left(Q_{t_{0}, t}\right)}^{2}+C_{0}\left\|\nabla \eta \varphi_{k}^{+}\right\|_{L^{2}\left(Q_{t_{0}, t}\right)}^{2},
\end{aligned}
$$


where $C_{0}>0$ depends on $\alpha_{0}$ and the $L^{4}\left(Q_{T^{\prime}, T}\right)$-norm of $\boldsymbol{u}$ only. For the final term on the right-hand side of (4.60), we employ Hölder's and Young's inequalities again to deduce that

$$
\begin{aligned}
\left|\int_{Q_{t_{0}, t}} \kappa(x, t) \cdot \nabla\left(\eta^{2} \varphi_{k}^{+}\right) d x d t\right| & =\left|\int_{Q_{t_{0}, t}}\left(\boldsymbol{\kappa}(x, t) \cdot \varphi_{k}^{+} \eta \nabla \eta+\eta \boldsymbol{\kappa}(x, t) \cdot \nabla\left(\eta \varphi_{k}^{+}\right)\right) d x d t\right| \\
& \leq C_{1} \int_{Q_{t_{0}, t}}|\eta|^{2} d x d t+\frac{1}{2} \int_{Q_{t_{0}, t}}\left(\varphi_{k}^{+}\right)^{2}|\nabla \eta|^{2} d x d t \\
& +\frac{\alpha_{0}}{4} \int_{Q_{t_{0}, t}}\left|\nabla\left(\eta \varphi_{k}^{+}\right)\right|^{2} d x d t,
\end{aligned}
$$

where $C_{1}>0$ depends only on $\alpha_{0}$ and the $L^{\infty}\left(Q_{T^{\prime}, T}\right)$-norm of $\boldsymbol{\kappa}$. Inserting the estimates (4.61) and (4.62) into the right-hand side of (4.60), we infer the existence of a constant $C_{2}=C_{2}\left(C_{0}, C_{1}, \lambda_{\infty}\right)>$ 0 such that

$$
\begin{aligned}
\frac{1}{2} \sup _{s \in\left(t_{0}, t\right)} & \int_{\Omega}\left(\eta \varphi_{k}^{+}\right)^{2}(s) d x+\alpha_{0} \int_{Q_{t_{0}, t}}\left|\nabla\left(\eta \varphi_{k}^{+}\right)\right|^{2} d x d t \leq \int_{\Omega}\left(\eta \varphi_{k}^{+}\right)^{2}\left(t_{0}\right) d x \\
& +C_{2}\left(\int_{Q_{t_{0}, t}}\left(\varphi_{k}^{+}\right)^{2}\left|\eta \eta_{t}\right| d x d t+\int_{Q_{t_{0}, t}}\left(\varphi_{k}^{+}\right)^{2}|\nabla \eta|^{2} d x d t+\int_{Q_{t_{0}, t}}|\eta|^{2} d x d t\right) .
\end{aligned}
$$

Arguing in a similar fashion, inequality (4.63) also holds with $\varphi$ replaced by $-\varphi$. In particular, these inequalities imply that $\varphi$ is an element of the class $\mathcal{B}_{2}\left(Q_{T^{\prime}, T}, 1, \gamma, 4,1,1\right)$ in the sense of [40, Chapter II, Section 7 ], for some $\gamma=\gamma\left(C_{2}\right)$, cf. inequality (7.5) of [40, Chapter II, Section 7, Remark 7.2]. Therefore, on account of [40, Chapter II, Section 7, Theorem 7.1], the Hölder continuity of $\varphi$ follows. This ends the proof.

The approximation argument that can be employed to show that

$$
\boldsymbol{u} \in L^{\infty}\left(0, T ; V_{\text {div }}\right) \cap L^{2}\left(0, T ; H^{2}(\Omega)^{2}\right)
$$

is the same as the one of [20, Proof of Theorem 5, Step 3], to which we refer for the details. We briefly recall the main points:

(a) $\varphi$ is suitably mollified in the viscosity term of the Navier-Stokes equation only, namely, the following problem is considered:

$$
\begin{aligned}
& \boldsymbol{u}_{t}-2 \operatorname{div}\left(\nu\left(\varphi_{\delta}\right) D \boldsymbol{u}\right)+(\boldsymbol{u} \cdot \nabla) \boldsymbol{u}+\nabla \pi=(-K * \varphi) \nabla \varphi+\boldsymbol{v} \\
& \operatorname{div}\left(\boldsymbol{u}_{\delta}\right)=0
\end{aligned}
$$

with initial condition $\boldsymbol{u}_{\delta}(0)=\boldsymbol{u}_{0}$ and no-slip boundary condition. Notice that, owing to the bound $|\varphi| \leq 1$, the approximation $\varphi_{\delta}$ of [20, Proof of Theorem 5, Step 3] satisfies $\left|\varphi_{\delta}\right| \leq 1$;

(b) The result of [1, Theorem 8] is then applied to get a strong local in time solution $\boldsymbol{u}_{\delta}$ to (4.64)-(4.65), such that

$$
\boldsymbol{u}_{\delta} \in H^{1}\left(0, T_{\delta} ; G_{d i v}\right) \cap L^{2}\left(0, T_{\delta} ; H^{2}(\Omega)^{2}\right) \cap L^{\infty}\left(0, T_{\delta} ; V_{d i v}\right),
$$

for some $T_{\delta} \leq T$;

(c) Thanks to Lemma 4.2 , we have $\nu\left(\varphi_{\delta}\right) \in C^{\gamma, \gamma / 2}(\bar{\Omega} \times[0, T])$, for some $0<\gamma \leq \min \{\alpha, \beta\}$, and this allows us to apply Proposition 4.1 to (4.57)-(4.58) (written with $\boldsymbol{u}_{\delta}$ and $\varphi_{\delta}$ in place of $\boldsymbol{u}$ and $\varphi$, respectively). Arguing as in [20, Proof of Theorem 5, Step 2], we test the Navier-Stokes equations (4.64) by $\partial_{t} \boldsymbol{u}_{\delta}$. It is then easy to deduce a differential inequality of 
the form

$$
\begin{aligned}
& \frac{d}{d t} \int_{\Omega} \nu\left(\varphi_{\delta}\right)\left|D \boldsymbol{u}_{\delta}\right|^{2}+\frac{1}{8}\left\|\partial_{t} \boldsymbol{u}_{\delta}\right\|^{2} \leq C\left(\left\|\boldsymbol{l}_{\delta}\right\|^{2}+\|\boldsymbol{v}\|^{2}+\left\|\nabla \varphi_{\delta}\right\|^{2}\right) \\
& +C\left(\left\|\boldsymbol{u}_{\delta}\right\|^{2}\left\|\nabla \boldsymbol{u}_{\delta}\right\|^{2}+\left\|\nabla \varphi_{\delta}\right\|_{L^{4}}^{4}+\left\|\partial_{t} \varphi_{\delta}\right\|^{2}\right)\left\|D \boldsymbol{u}_{\delta}\right\|^{2}
\end{aligned}
$$

where

$$
\boldsymbol{l}_{\delta}:=-\left(K * \varphi_{\delta}\right) \nabla \varphi_{\delta}+\boldsymbol{v} .
$$

We recall here below the main arguments of [20, Proof of Theorem 5, Step 2].

- One can exploit (4.66), assumption (V) and the improved regularity for $\varphi$ obtained in Step 1, as well as the fact that we have $\varphi \in L^{4}\left(0, T ; W^{1,4}(\Omega)\right)$. These regularity properties yield that $\partial_{t} \varphi_{\delta}$ is bounded in $L^{2}(0, T ; H)$ and that $\varphi_{\delta}$ is bounded in $L^{4}\left(0, T ; W^{1,4}(\Omega)\right)$ uniformly with respect to $\delta$.

- We can exploit the uniform with respect to $\delta$ bound of $\boldsymbol{u}_{\delta}$ in the space $L^{\infty}\left(0, T ; G_{d i v}\right) \cap$ $L^{2}\left(0, T ; V_{d i v}\right)$, which stems from the energy identity obtained by testing (4.64) by $\boldsymbol{u}_{\delta}$ in $G_{d i v}$, and the fact that $\boldsymbol{u}_{0} \in V_{d i v}$.

- Using Gronwall's lemma and Proposition 4.1, we can prove that $\boldsymbol{u}_{\delta}$ is bounded in $L^{\infty}\left(0, T_{\delta} ; V_{d i v}\right) \cap H^{1}\left(0, T_{\delta} ; G_{d i v}\right)$ uniformly with respect to $\delta$, and, by comparison in (4.64), that $\boldsymbol{u}_{\delta}$ is uniformly bounded in $L^{2}\left(0, T_{\delta} ; H^{2}(\Omega)^{2}\right)$. These estimates entail, in particular, that $\boldsymbol{u}_{\delta}$ can be extended to any time interval $(0, T)$, for all $T>0$;

(d) The passage to the limit in (4.64)-(4.65), as $\delta \rightarrow 0$, is performed, by employing compactness arguments and the strong convergence $\varphi_{\delta}(t) \rightarrow \varphi$ in $V$, for almost any $t \in(0, T)$. This gives a strong solution $\widetilde{\boldsymbol{u}}$ to the same problem solved by the weak solution $\boldsymbol{u}$. Finally, the limit velocity field satisfies $\widetilde{\boldsymbol{u}}=\boldsymbol{u}$, on account of the uniqueness for the Navier-Stokes equation with a given (nonconstant) viscosity. Therefore, the existence of a strong solution satisfying (3.9) and (3.10) is proved. The uniqueness of this strong solution follows from [20, Theorem 7]. This concludes the proof of the second part of Theorem 3.6.

Step 3. In order to prove the last regularity part in (3.12), the idea is to differentiate (3.1) with respect to time and to test the resulting equation by $\varphi_{t}$. To make the argument rigorous, we employ the same time-discretization scheme of Step 1, taking the improved regularity for $\boldsymbol{u}$ (cf. Step 2) into account. Therefore, for $k=1, \ldots, N-1$, we consider problem (4.9)-(4.10) (where, in (4.9), the discrete time derivative $\left(\varphi_{k+1}-\varphi_{k}\right) / \tau$ is made explicit) at step $k$ and at step $k-1$. Taking the difference between the two equations (4.9) written for these steps, testing the resulting identity by $\left(\varphi_{k+1}-\varphi_{k}\right) / \tau$, and summing over $k=1, \ldots, n$, with $n \leq N-1$, we obtain the identity

$$
\begin{aligned}
& \sum_{k=1}^{n}\left(\frac{\varphi_{k+1}-\varphi_{k}}{\tau}-\frac{\varphi_{k}-\varphi_{k-1}}{\tau}, \frac{\varphi_{k+1}-\varphi_{k}}{\tau}\right) \\
& =-\sum_{k=1}^{n}\left(\nabla\left(B\left(\varphi_{k+1}\right)-B\left(\varphi_{k}\right)\right), \nabla\left(\frac{\varphi_{k+1}-\varphi_{k}}{\tau}\right)\right) \\
& -\sum_{k=1}^{n}\left(\boldsymbol{U}_{k} \cdot \nabla \varphi_{k+1}-\boldsymbol{U}_{k-1} \cdot \nabla \varphi_{k}, \frac{\varphi_{k+1}-\varphi_{k}}{\tau}\right) \\
& +\sum_{k=1}^{n}\left(m\left(\varphi_{k}\right)\left(\nabla K * Q\left(\varphi_{k}\right)\right)-m\left(\varphi_{k-1}\right)\left(\nabla K * Q\left(\varphi_{k-1}\right)\right), \nabla\left(\frac{\varphi_{k+1}-\varphi_{k}}{\tau}\right)\right),
\end{aligned}
$$

where, again, for simplicity of notation, the explicit indication of the parameter $\epsilon$ is omitted. 
Let us now estimate the terms on the right-hand side of (4.67). As far as the first term is concerned, we obtain that

$$
\begin{aligned}
& \sum_{k=1}^{n}\left(\nabla\left(B\left(\varphi_{k+1}\right)-B\left(\varphi_{k}\right)\right), \nabla\left(\frac{\varphi_{k+1}-\varphi_{k}}{\tau}\right)\right) \\
& =\tau \sum_{k=1}^{n}\left(\lambda\left(\varphi_{k+1}\right) \nabla\left(\frac{\varphi_{k+1}-\varphi_{k}}{\tau}\right), \nabla\left(\frac{\varphi_{k+1}-\varphi_{k}}{\tau}\right)\right) \\
& +\sum_{k=1}^{n}\left(\left(\lambda\left(\varphi_{k+1}\right)-\lambda\left(\varphi_{k}\right)\right) \nabla \varphi_{k}, \nabla\left(\frac{\varphi_{k+1}-\varphi_{k}}{\tau}\right)\right) \\
& \geq \frac{1}{2} \alpha_{0} \tau \sum_{k=1}^{n}\left\|\nabla\left(\frac{\varphi_{k+1}-\varphi_{k}}{\tau}\right)\right\|^{2} \\
& -\frac{\tau}{2 \alpha_{0}} \sum_{k=1}^{n}\left\|\frac{\lambda\left(\varphi_{k+1}\right)-\lambda\left(\varphi_{k}\right)}{\tau} \nabla \varphi_{k}\right\|^{2} .
\end{aligned}
$$

On the other hand, in light of (4.8), (4.31) and the fact that $\nabla B(\varphi)=\lambda(\varphi) \nabla \varphi$, we have that

$$
\begin{aligned}
& \left\|\frac{\lambda\left(\varphi_{k+1}\right)-\lambda\left(\varphi_{k}\right)}{\tau} \nabla \varphi_{k}\right\|^{2} \\
& \leq \lambda_{\infty}^{\prime}{ }^{2}\left\|\frac{\varphi_{k+1}-\varphi_{k}}{\tau}\right\|_{L^{4}(\Omega)}^{2}\left\|\nabla \varphi_{k}\right\|_{L^{4}(\Omega)^{2}}^{2} \\
& \leq C\left(\left\|\frac{\varphi_{k+1}-\varphi_{k}}{\tau}\right\|^{2}+\left\|\frac{\varphi_{k+1}-\varphi_{k}}{\tau}\right\|\right. \\
& \left.\times\left\|\nabla\left(\frac{\varphi_{k+1}-\varphi_{k}}{\tau}\right)\right\|\right)\left\|\frac{\nabla B\left(\varphi_{k}\right)}{\lambda\left(\varphi_{k}\right)}\right\|_{L^{4}(\Omega)^{2}}^{2} \\
& \leq \frac{\alpha_{0}}{2}\left\|\nabla\left(\frac{\varphi_{k+1}-\varphi_{k}}{\tau}\right)\right\|^{2} \\
& +C\left\|B\left(\varphi_{k}\right)\right\|_{H^{2}(\Omega)}^{2}\left\|\frac{\varphi_{k+1}-\varphi_{k}}{\tau}\right\|^{2}+C\left\|\frac{\varphi_{k+1}-\varphi_{k}}{\tau}\right\|^{2} .
\end{aligned}
$$

Therefore, we conclude the estimate

$$
\begin{aligned}
& \sum_{k=1}^{n}\left(\nabla\left(B\left(\varphi_{k+1}\right)-B\left(\varphi_{k}\right)\right), \nabla\left(\frac{\varphi_{k+1}-\varphi_{k}}{\tau}\right)\right) \\
& \geq \frac{\alpha_{0} \tau}{4} \sum_{k=1}^{n}\left\|\nabla\left(\frac{\varphi_{k+1}-\varphi_{k}}{\tau}\right)\right\|^{2} \\
& -C \tau \sum_{k=1}^{n}\left\|B\left(\varphi_{k}\right)\right\|_{H^{2}(\Omega)}^{2}\left\|\frac{\varphi_{k+1}-\varphi_{k}}{\tau}\right\|^{2} \\
& -C \tau \sum_{k=1}^{n}\left\|\frac{\varphi_{k+1}-\varphi_{k}}{\tau}\right\|^{2} .
\end{aligned}
$$


Regarding the second term on the right-hand side of (4.67), we have

$$
\begin{aligned}
& \sum_{k=1}^{n}\left(\boldsymbol{U}_{k} \cdot \nabla \varphi_{k+1}-\boldsymbol{U}_{k-1} \cdot \nabla \varphi_{k}, \frac{\varphi_{k+1}-\varphi_{k}}{\tau}\right) \\
& =\tau \sum_{k=1}^{n}\left(\frac{\boldsymbol{U}_{k}-\boldsymbol{U}_{k-1}}{\tau} \cdot \nabla \varphi_{k}, \frac{\varphi_{k+1}-\varphi_{k}}{\tau}\right) \\
& \leq \tau \sum_{k=1}^{n}\left\|\frac{\boldsymbol{U}_{k}-\boldsymbol{U}_{k-1}}{\tau}\right\|\left\|\frac{\varphi_{k+1}-\varphi_{k}}{\tau}\right\|_{L^{4}(\Omega)}\left\|\frac{\nabla B\left(\varphi_{k}\right)}{\lambda\left(\varphi_{k}\right)}\right\|_{L^{4}(\Omega)^{2}} \\
& \leq \tau C \sum_{k=1}^{n}\left\|\frac{\boldsymbol{U}_{k}-\boldsymbol{U}_{k-1}}{\tau}\right\|\left(\left\|\frac{\varphi_{k+1}-\varphi_{k}}{\tau}\right\|\right. \\
& \left.+\left\|\frac{\varphi_{k+1}-\varphi_{k}}{\tau}\right\|^{1 / 2}\left\|\nabla\left(\frac{\varphi_{k+1}-\varphi_{k}}{\tau}\right)\right\|^{1 / 2}\right)\left\|B\left(\varphi_{k}\right)\right\|_{H^{2}(\Omega)}^{1 / 2} \\
& \leq \delta \tau \sum_{k=1}^{n}\left\|\nabla\left(\frac{\varphi_{k+1}-\varphi_{k}}{\tau}\right)\right\|^{2}+C_{\delta} \tau \sum_{k=1}^{n}\left\|\frac{U_{k}-\boldsymbol{U}_{k-1}}{\tau}\right\|^{2} \\
& +C_{\delta} \tau \sum_{k=1}^{n}\left\|B\left(\varphi_{k}\right)\right\|_{H^{2}(\Omega)}^{2}\left\|\frac{\varphi_{k+1}-\varphi_{k}}{\tau}\right\|^{2}+C_{\delta} \tau \sum_{k=1}^{n}\left\|\frac{\varphi_{k+1}-\varphi_{k}}{\tau}\right\|^{2},
\end{aligned}
$$

where $\delta>0$ will be fixed later. Finally, the last term on the right-hand side of (4.67) is estimated as follows:

$$
\begin{aligned}
& \sum_{k=1}^{n}\left(m\left(\varphi_{k}\right)\left(\nabla K * Q\left(\varphi_{k}\right)\right)-m\left(\varphi_{k-1}\right)\left(\nabla K * Q\left(\varphi_{k-1}\right)\right), \nabla\left(\frac{\varphi_{k+1}-\varphi_{k}}{\tau}\right)\right) \\
& \leq\left(m_{\infty}+m_{\infty}^{\prime}\right) b \tau \sum_{k=1}^{n}\left\|\frac{\varphi_{k}-\varphi_{k-1}}{\tau}\right\|\left\|\nabla\left(\frac{\varphi_{k+1}-\varphi_{k}}{\tau}\right)\right\| \\
& \leq \delta \tau \sum_{k=1}^{n}\left\|\nabla\left(\frac{\varphi_{k+1}-\varphi_{k}}{\tau}\right)\right\|^{2}+C_{\delta} \tau \sum_{k=1}^{n}\left\|\frac{\varphi_{k}-\varphi_{k-1}}{\tau}\right\|^{2}
\end{aligned}
$$

By applying (4.14) (with $\left(\varphi_{k}-\varphi_{k-1}\right) / \tau$ in place of $\left.\varphi_{k}\right)$ to the left-hand side of (4.67), inserting estimates (4.71)-(4.73) into the right-hand side, choosing $\delta$ small enough, and taking (4.31) into account, we obtain that

$$
\begin{aligned}
& \frac{1}{2}\left\|\frac{\varphi_{n+1}-\varphi_{n}}{\tau}\right\|^{2}+\frac{1}{2} \sum_{k=1}^{n}\left\|\frac{\varphi_{k+1}-\varphi_{k}}{\tau}-\frac{\varphi_{k}-\varphi_{k-1}}{\tau}\right\|^{2} \\
& +\frac{1}{8} \alpha_{0} \tau \sum_{k=1}^{n}\left\|\nabla\left(\frac{\varphi_{k+1}-\varphi_{k}}{\tau}\right)\right\|^{2} \\
& \leq \frac{1}{2}\left\|\frac{\varphi_{1}-\varphi_{0}}{\tau}\right\|^{2}+C \tau \sum_{k=1}^{n}\left\|B\left(\varphi_{k}\right)\right\|_{H^{2}(\Omega)}^{2}\left\|\frac{\varphi_{k+1}-\varphi_{k}}{\tau}\right\|^{2} \\
& +C \tau \sum_{k=1}^{n}\left\|\frac{\boldsymbol{U}_{k}-\boldsymbol{U}_{k-1}}{\tau}\right\|^{2}+C .
\end{aligned}
$$


Observe now that we have (cf. (4.24)-(4.29) and (4.31))

$$
\tau\left\|B\left(\varphi_{k}\right)\right\|_{H^{2}(\Omega)}^{2} \leq \frac{C}{\tau}\left\|\varphi_{k}-\varphi_{k-1}\right\|^{2}+C \tau
$$

where here the constant $C$ depends on the norm of $\boldsymbol{u}$ in $L^{\infty}\left(0, T ; G_{d i v}\right) \cap L^{2}\left(0, T ; V_{d i v}\right)$. Therefore, we can infer from (4.74) that

$$
\begin{aligned}
& \frac{1}{2}\left\|\frac{\varphi_{n+1}-\varphi_{n}}{\tau}\right\|^{2}+\frac{1}{2} \sum_{k=1}^{n}\left\|\frac{\varphi_{k+1}-\varphi_{k}}{\tau}-\frac{\varphi_{k}-\varphi_{k-1}}{\tau}\right\|^{2} \\
& +\frac{1}{8} \alpha_{0} \tau \sum_{k=1}^{n}\left\|\nabla\left(\frac{\varphi_{k+1}-\varphi_{k}}{\tau}\right)\right\|^{2} \\
& \leq \frac{1}{2}\left\|\frac{\varphi_{1}-\varphi_{0}}{\tau}\right\|^{2}+C \sum_{k=0}^{n-1} \frac{1}{\tau}\left\|\varphi_{k+2}-\varphi_{k+1}\right\|^{2}\left\|\frac{\varphi_{k+1}-\varphi_{k}}{\tau}\right\|^{2} \\
& +\frac{C}{\tau} \sum_{k=1}^{n}\left\|\varphi_{k+1}-\varphi_{k}\right\|^{2}+C \tau \sum_{k=1}^{n}\left\|\frac{\boldsymbol{U}_{k}-\boldsymbol{U}_{k-1}}{\tau}\right\|^{2}+C .
\end{aligned}
$$

The delicate point is now the control of the $L^{2}$-norm of the quotient $\left(\varphi_{1}-\varphi_{0}\right) / \tau$ on the righthand side. To this goal, let us first point out a remarkable consequence we have from the improved regularity of the velocity field obtained in Step 2, which concerns the solvability of the incrementalstep problem (4.9)-(4.10). Indeed, for a given $\varphi_{k} \in V, k=0, \ldots, N-1$, let us introduce the nonlinear operator $\mathcal{B}_{k}: D\left(\mathcal{B}_{k}\right) \subset H \rightarrow H$, defined by

$$
\begin{aligned}
& \mathcal{B}_{k} \varphi:=-\Delta B(\varphi)+\boldsymbol{U}_{k} \cdot \nabla \varphi+\operatorname{div}\left(m\left(\varphi_{k}\right)\left(\nabla K * Q\left(\varphi_{k}\right)\right)\right), \\
& D\left(\mathcal{B}_{k}\right):=\left\{\varphi \in H^{2}(\Omega): \nabla B(\varphi) \cdot \boldsymbol{n}=m\left(\varphi_{k}\right)\left(\nabla K * Q\left(\varphi_{k}\right)\right) \cdot \boldsymbol{n}, \text { a.e. on } \partial \Omega\right\} .
\end{aligned}
$$

We prove that there exists some $\tau_{0}=\tau_{0}(\boldsymbol{u})>0$ such that

$$
\left\|\left(\varphi_{2}-\varphi_{1}\right)+\tau\left(\mathcal{B}_{k} \varphi_{2}-\mathcal{B}_{k} \varphi_{1}\right)\right\| \geq \frac{\alpha_{0}}{2 \lambda_{\infty}}\left\|\varphi_{2}-\varphi_{1}\right\|, \quad \forall \varphi_{1}, \varphi_{2} \in D\left(\mathcal{B}_{k}\right), 0<\tau \leq \tau_{0}
$$

This estimate, in particular, implies that the solution to each incremental-step problem (4.9)-(4.10), for $k=0, \ldots, N-1$, is unique.

In order to show (4.76), we first observe that, for all $\varphi_{1}, \varphi_{2} \in D\left(\mathcal{B}_{k}\right)$, we have

$$
\begin{aligned}
& \left(\varphi_{2}-\varphi_{1}+\tau\left(\mathcal{B}_{k} \varphi_{2}-\mathcal{B}_{k} \varphi_{1}\right), B\left(\varphi_{2}\right)-B\left(\varphi_{1}\right)\right) \geq \alpha_{0}\left\|\varphi_{2}-\varphi_{1}\right\|^{2} \\
& +\tau\left\|\nabla\left(B\left(\varphi_{2}\right)-B\left(\varphi_{1}\right)\right)\right\|^{2}-\tau\left(\boldsymbol{U}_{k} \cdot\left(\varphi_{2}-\varphi_{1}\right), \nabla\left(B\left(\varphi_{2}\right)-B\left(\varphi_{1}\right)\right)\right) .
\end{aligned}
$$

Thanks to the improved regularity (3.10), it holds that

$$
\left\|\boldsymbol{U}_{k}\right\|_{V_{\text {div }}} \leq\|\boldsymbol{u}\|_{L^{\infty}\left(0, T ; V_{\text {div }}\right)}, \quad\left\|\boldsymbol{U}_{k}\right\|_{H^{2}(\Omega)^{2}} \leq \frac{1}{\sqrt{\tau}}\|\boldsymbol{u}\|_{L^{2}\left(0, T ; H^{2}(\Omega)^{2}\right)}
$$


Hence, by means of (4.78) 2 and by Agmon's inequality (2.2), the last term on the right-hand side of (4.77) can be estimated as follows:

$$
\begin{aligned}
& \tau\left|\left(\boldsymbol{U}_{k} \cdot\left(\varphi_{2}-\varphi_{1}\right), \nabla\left(B\left(\varphi_{2}\right)-B\left(\varphi_{1}\right)\right)\right)\right| \\
& \leq \tau\left\|\boldsymbol{U}_{k}\right\|_{L^{\infty}(\Omega)^{2}}\left\|\varphi_{2}-\varphi_{1}\right\|\left\|\nabla\left(B\left(\varphi_{2}\right)-B\left(\varphi_{1}\right)\right)\right\| \\
& \leq \frac{\tau}{2}\left\|\nabla\left(B\left(\varphi_{2}\right)-B\left(\varphi_{1}\right)\right)\right\|^{2}+\frac{\tau}{2} \hat{C}_{3}^{2}\left\|\boldsymbol{U}_{k}\right\|\left\|\boldsymbol{U}_{k}\right\|_{H^{2}(\Omega)^{2}}\left\|\varphi_{2}-\varphi_{1}\right\|^{2} \\
& \leq \frac{\tau}{2}\left\|\nabla\left(B\left(\varphi_{2}\right)-B\left(\varphi_{1}\right)\right)\right\|^{2}+\frac{\sqrt{\tau}}{2} \hat{C}_{3}^{2}\|\boldsymbol{u}\|_{L^{\infty}\left(0, T ; G_{d i v}\right)}\|\boldsymbol{u}\|_{L^{2}\left(0, T ; H^{2}(\Omega)^{2}\right)}\left\|\varphi_{2}-\varphi_{1}\right\|^{2} .
\end{aligned}
$$

Therefore, taking $0<\tau \leq \tau_{0}$ with $\tau_{0}$ given by

$$
\tau_{0}:=\frac{\alpha_{0}^{2}}{\hat{C}_{3}^{4}\|\boldsymbol{u}\|_{L^{\infty}\left(0, T ; G_{d i v}\right)}^{2}\|\boldsymbol{u}\|_{L^{2}\left(0, T ; H^{2}(\Omega)^{2}\right)}^{2}},
$$

the right-hand side of (4.77) can be bounded from below by

$$
\frac{\alpha_{0}}{2}\left\|\varphi_{2}-\varphi_{1}\right\|^{2}+\frac{\tau}{2}\left\|\nabla\left(B\left(\varphi_{2}\right)-B\left(\varphi_{1}\right)\right)\right\|^{2} .
$$

On the other hand, due to (4.5), we have that

$$
\begin{aligned}
& \left(\varphi_{2}-\varphi_{1}+\tau\left(\mathcal{B}_{k} \varphi_{2}-\mathcal{B}_{k} \varphi_{1}\right), B\left(\varphi_{2}\right)-B\left(\varphi_{1}\right)\right) \\
& \leq \lambda_{\infty}\left\|\varphi_{2}-\varphi_{1}+\tau\left(\mathcal{B}_{k} \varphi_{2}-\mathcal{B}_{k} \varphi_{1}\right)\right\|\left\|\varphi_{2}-\varphi_{1}\right\| \\
& \leq \frac{\alpha_{0}}{4}\left\|\varphi_{2}-\varphi_{1}\right\|^{2}+\frac{\lambda_{\infty}^{2}}{\alpha_{0}}\left\|\varphi_{2}-\varphi_{1}+\tau\left(\mathcal{B}_{k} \varphi_{2}-\mathcal{B}_{k} \varphi_{1}\right)\right\|^{2} .
\end{aligned}
$$

Hence, from (4.77), (4.80), and (4.81), we get the estimate

$$
\frac{\lambda_{\infty}^{2}}{\alpha_{0}}\left\|\varphi_{2}-\varphi_{1}+\tau\left(\mathcal{B}_{k} \varphi_{2}-\mathcal{B}_{k} \varphi_{1}\right)\right\|^{2} \geq \frac{\alpha_{0}}{4}\left\|\varphi_{2}-\varphi_{1}\right\|^{2}+\frac{\tau}{2}\left\|\nabla\left(B\left(\varphi_{2}\right)-B\left(\varphi_{1}\right)\right)\right\|^{2} .
$$

This proves the desired claim (4.76). Therefore, for $0<\tau \leq \tau_{0}$ and for every $k=0, \ldots, N-1$, the resolvent operator $J_{k, \tau}:=\left(I+\tau \mathcal{B}_{k}\right)^{-1}$ is single-valued and Lipschitz continuous from $H$ to $H$. Indeed, it holds

$$
\left\|J_{k, \tau} \psi_{2}-J_{k, \tau} \psi_{1}\right\| \leq \frac{2 \lambda_{\infty}}{\alpha_{0}}\left\|\psi_{2}-\psi_{1}\right\|, \quad \forall \psi_{1}, \psi_{2} \in H, \quad 0<\tau \leq \tau_{0} .
$$

Notice that, if the first term $\varphi_{k}$ on the right-hand side of (4.9) is assumed in $H$, then the solvability of problem (4.9)-(4.10) still holds true, arguing as at the beginning of Step 1. Indeed, the nonlinear operator $A_{k}$ is the same and we still have $g_{k} \in V^{\prime}$.

Let us now go back to the problem of controlling the $L^{2}$-norm of the quotient $\left(\varphi_{1}-\varphi_{0}\right) / \tau$. By employing (4.82) for $k=0$, using the assumption on $\varphi_{0}$ which yields that $\varphi_{0} \in D\left(\mathcal{B}_{0}\right)$, and assuming that $0<\tau \leq \tau_{0}$, we find that

$$
\begin{aligned}
& \left\|\frac{\varphi_{1}-\varphi_{0}}{\tau}\right\|=\left\|\frac{J_{0, \tau} \varphi_{0}-J_{0, \tau}\left(I+\tau \mathcal{B}_{0}\right) \varphi_{0}}{\tau}\right\| \\
& \leq \frac{2 \lambda_{\infty}}{\alpha_{0}}\left\|\mathcal{B}_{0} \varphi_{0}\right\| \\
& \leq C\left(\left\|\Delta B\left(\varphi_{0}\right)\right\|+\|\boldsymbol{u}\|_{L^{\infty}\left(0, T ; V_{\text {div }}\right)}\left\|\varphi_{0}\right\|_{H^{2}(\Omega)}+\left\|\varphi_{0}\right\|_{V}+1\right),
\end{aligned}
$$

where we have also used the first of (4.78). 
Finally, there remains to bound the last summand on the right-hand side of (4.74). To this end, we can first observe that the following estimate holds true:

$$
\left\|\boldsymbol{u}(k \tau)-\boldsymbol{U}_{k}\right\|^{2} \leq \frac{\tau}{3} \int_{k \tau}^{(k+1) \tau}\left\|\boldsymbol{u}_{t}(s)\right\|^{2} d s .
$$

This estimate and a simple computation yield that

$$
\frac{1}{\tau} \sum_{k=1}^{n}\left\|\boldsymbol{U}_{k}-\boldsymbol{U}_{k-1}\right\|^{2} \leq c\left\|\boldsymbol{u}_{t}\right\|_{L^{2}\left(0, T ; G_{d i v}\right)}^{2},
$$

where the constant $c>0$ can be given by $c=10 / 3$.

We can now apply the discrete Gronwall's Lemma to (4.74), taking (4.31), (4.83) and (4.84) into account, to obtain the estimate

$$
\begin{aligned}
& \left\|\frac{\varphi_{n+1}-\varphi_{n}}{\tau}\right\|^{2}+\tau \sum_{k=1}^{n}\left\|\nabla\left(\frac{\varphi_{k+1}-\varphi_{k}}{\tau}\right)\right\|^{2} \\
& \leq \mathbb{Q}\left(\left\|\varphi_{0}\right\|_{H^{2}(\Omega)},\|\boldsymbol{u}\|_{L^{\infty}\left(0, T ; V_{d i v}\right) \cap H^{1}\left(0, T ; G_{d i v}\right)}\right) .
\end{aligned}
$$

From this discrete estimate, we get a new bound for the approximate solutions $\widehat{\varphi}_{N}, \bar{\varphi}_{N}$ introduced in Step 1. Namely, we have

$$
\left\|\widehat{\varphi}_{N}^{\prime}\right\|_{L^{\infty}(0, T ; H)}^{2}+\left\|\widehat{\varphi}_{N}^{\prime}\right\|_{L^{2}(0, T ; V)}^{2} \leq \mathbb{Q}\left(\left\|\varphi_{0}\right\|_{H^{2}(\Omega)},\|\boldsymbol{u}\|_{L^{\infty}\left(0, T ; V_{d i v}\right) \cap H^{1}\left(0, T ; G_{d i v}\right)}\right) .
$$

Therefore, in addition to (4.41)-(4.48), we also have, at least for a subsequence, that

$$
\widehat{\varphi}_{N}^{\prime} \rightarrow \varphi_{t}, \quad \text { weakly-star in } L^{\infty}(0, T ; H), \quad \text { weakly in } L^{2}(0, T ; V) .
$$

This proves the second part of (3.12). Moreover, since we have (cf. (4.24)-(4.29) and (4.31))

$$
\left\|B\left(\varphi_{n+1}\right)\right\|_{H^{2}(\Omega)}^{2} \leq C\left\|\frac{\varphi_{n+1}-\varphi_{n}}{\tau}\right\|^{2}+C
$$

then, thanks to (4.85), we also get the bound

$$
\left\|B\left(\bar{\varphi}_{N}\right)\right\|_{L^{\infty}\left(0, T ; H^{2}(\Omega)\right)} \leq \mathbb{Q}\left(\left\|\varphi_{0}\right\|_{H^{2}(\Omega)},\|\boldsymbol{u}\|_{L^{\infty}\left(0, T ; V_{d i v}\right) \cap H^{1}\left(0, T ; G_{d i v}\right)}\right) .
$$

This obviously implies that

$$
\begin{aligned}
& \left\|\bar{\varphi}_{N}\right\|_{L^{\infty}\left(0, T ; W^{1, p}(\Omega)\right)}+\left\|B\left(\bar{\varphi}_{N}\right)\right\|_{L^{\infty}\left(0, T ; W^{1, p}(\Omega)\right)}+\left\|\lambda\left(\bar{\varphi}_{N}\right)\right\|_{L^{\infty}\left(0, T ; W^{1, p}(\Omega)\right)} \\
& \leq \mathbb{Q}\left(\left\|\varphi_{0}\right\|_{H^{2}(\Omega)},\|\boldsymbol{u}\|_{L^{\infty}\left(0, T ; V_{d i v}\right) \cap H^{1}\left(0, T ; G_{d i v}\right)}\right) .
\end{aligned}
$$

Hence, recalling (4.13) (written in terms of the approximate solutions $\bar{\varphi}_{N}$ ), and using (4.86), we infer that

$$
\left\|\bar{\varphi}_{N}\right\|_{L^{\infty}\left(0, T ; H^{2}(\Omega)\right)} \leq \mathbb{Q}\left(\left\|\varphi_{0}\right\|_{H^{2}(\Omega)},\|\boldsymbol{u}\|_{L^{\infty}\left(0, T ; V_{d i v}\right) \cap H^{1}\left(0, T ; G_{d i v}\right)}\right) .
$$

Therefore, at least for a subsequence, we have that

$$
\bar{\varphi}_{N} \rightarrow \varphi, \quad \text { weakly-star in } L^{\infty}\left(0, T ; H^{2}(\Omega)\right),
$$

whence we get the first part of (3.12). The argument to pass to the limit in (4.38)-(4.39), and also to prove the pointwise bound $|\varphi| \leq 1$, is the same as in Step 1 (indeed, here we can even rely on stronger convergence results). The proof of Theorem 3.6 is finished.

Remark 4.3. It is not known whether a strong solution according to Definition 3.1 also satisfies equations (1.2)-(1.3) and the related boundary condition in a strong sense. This occurs if we can guarantee the validity of a strict separation property, namely, the fact that $\varphi$ stays uniformly away from the pure phases (see, e.g., $[38,39]$ for a slightly different version of a nonlocal Cahn-Hilliard 
equation). An intermediate situation holds if $F^{\prime}\left(\varphi_{0}\right) \in H$ (see [24, Theorem 3]). In this case, the weak formulation where $\mu \in L^{2}(0, T ; V)$ appears explicitly can be recovered (cf. [24, Definition 1]).

Remark 4.4. If $\nu$ is constant, then the argument to get the existence of strong solutions to (1.1)(1.5) can be simplified. Indeed, one may exploit the classical regularity result of [46, Theorem 3.10] for the two-dimensional Navier-Stokes system. This was the strategy followed in [23, Proof of Theorem 2]. Notice first that (1.1) can be rewritten in the form

$$
\boldsymbol{u}_{t}-\nu \Delta \boldsymbol{u}+(\boldsymbol{u} \cdot \nabla) \boldsymbol{u}+\nabla \hat{\pi}=(a \varphi-K * \varphi) \nabla \varphi+\boldsymbol{v},
$$

where the modified pressure $\hat{\pi}:=\pi-F(\varphi)$ has been introduced. Thanks to the regularity properties of the weak solution (cf., in particular, the bound $|\varphi| \leq 1$ ) and to the assumption on $\boldsymbol{v}$, we see that the right-hand side of (4.88) belongs to $L^{2}\left(0, T ; L^{2}(\Omega)^{2}\right)$. Hence, under the assumption that $\boldsymbol{u}_{0} \in V_{\text {div }}$, the regularity (3.8) for the velocity field $\boldsymbol{u}$ immediately follows from applying [46, Theorem 3.10] to (4.88). Once (3.8) is available, we can devise an easier argument in Step 1, by using (4.22) and the second part of (4.78), to estimate the last term on the right-hand side of (4.18) as follows:

$$
\begin{aligned}
\left|\sum_{k=0}^{n}\left(\boldsymbol{U}_{k} \cdot \nabla \varphi_{k+1}, B\left(\cdot, \varphi_{k+1}\right)-B\left(\cdot, \varphi_{k}\right)\right)\right| & \leq \frac{\alpha_{0}}{4 \tau} \sum_{k=0}^{n}\left\|\varphi_{k+1}-\varphi_{k}\right\|^{2} \\
& +C \lambda_{\infty}^{2}\|\boldsymbol{u}\|_{L^{2}\left(0, T ; H^{2}(\Omega)^{2}\right)}^{2} \sum_{k=0}^{n}\left\|\nabla \varphi_{k+1}\right\|^{2} .
\end{aligned}
$$

This estimate, together with (4.19) and (4.20), still yields a discrete Gronwall's inequality from (4.18) (cf. (4.30)) and thus allows to obtain the regularity $\varphi \in L^{\infty}(0, T ; V), \varphi_{t} \in L^{2}(0, T ; H)$. Notice that the assumption that $K \in W_{l o c}^{2,1}$ or that $K$ is admissible is not required in this argument (only $(\mathbf{K})$ is enough). This regularity assumption on the kernel is needed only in Step 3, in order to prove that $\varphi \in L^{2}\left(0, T ; H^{2}(\Omega)\right)$ and, provided $\varphi_{0} \in H^{2}(\Omega)$ satisfies (3.11), that (3.12) holds.

Remark 4.5. Assume that $\boldsymbol{u}_{0} \in V_{\text {div }}$ and that $\varphi_{0} \in H^{2}(\Omega)$ satisfies (3.11). By integrating (4.66) in time, and by passing to the liminf in (4.36), (4.87), we can also prove that there exists a continuous and nondecreasing function $\overline{\mathbb{Q}}:[0, \infty) \rightarrow[0,+\infty)$ which only depends on the data $F, m, K, \nu, \Omega$, $T, \boldsymbol{u}_{0}$ and $\varphi_{0}$, such that

$$
\begin{aligned}
& \|\boldsymbol{u}\|_{L^{\infty}\left([0, T] ; V_{d i v}\right) \cap L^{2}\left(0, T ; H^{2}(\Omega)^{2}\right)}+\left\|\boldsymbol{u}_{t}\right\|_{L^{2}\left([0, T] ; G_{d i v}\right)}+\|\varphi\|_{L^{\infty}\left([0, T] ; H^{2}(\Omega)\right)} \\
& \quad+\left\|\varphi_{t}\right\|_{L^{\infty}([0, T] ; H) \cap L^{2}(0, T ; V)} \\
& \leq \overline{\mathbb{Q}}\left(\|\boldsymbol{v}\|_{L^{2}\left(0, T ; G_{d i v}\right)}\right) .
\end{aligned}
$$

Remark 4.6. We point out that the estimates in the proof of Theorem 3.6 rely essentially on:

(a) the boundedness and Lipschitz continuity properties of the nonlinear functions $\lambda, m$, given by (4.5)-(4.8);

(b) the fact that $\varphi$ is bounded (cf. the control of the boundary term in (4.25)).

Therefore, the whole strategy developed in the proof of Theorem 3.6 also applies for other classes of mobilities and double-well potentials, provided that the previous two points are valid. An example is given by a nondegenerate mobility and a regular potential defined instead on $\mathbb{R}$, satisfying the assumptions of [23, Theorem 2]. The boundedness of $\varphi$ follows from a strategy based on a Moser iteration argument (see [8, Theorem 2.1]). More precisely, in this case the uniform $L^{\infty}(\Omega)$-bound of $\varphi_{k+1}$ (cf. Step I of the proof of Theorem 3.6) will be given below (cf. the proof of Theorem 6.1 ). Incidentally, we point out that the present strategy is an example of how the argument used in the proof [23, Theorem 5] can be made rigorous. 


\section{UNIFORM ESTIMATES}

In this section, we establish some uniform in time regularization estimates. To this aim, we shall first formally deduce the same kind of higher-order bounds which were derived rigorously in the context of the time-discretization scheme in the proof of Theorem 3.6. These will be the basis for constructing uniform in time estimates. As a consequence, we establish a regularity property for the global attractor of the dynamical system generated by (1.1)-(1.6), the existence of which was proven in [24]. We point out that the argument of Proposition 5.1 below can be made rigorous by means of time discretization combined with a discrete variant of the uniform Gronwall's lemma (see [43, Lemma 3]). Thus, we proceed formally, just for the sake of brevity.

Proposition 5.1. Suppose that assumptions (K), (V), (M), (A1b), (A4) are satisfied, and suppose that $K \in W_{\text {loc }}^{2,1}\left(\mathbb{R}^{2}\right)$ or that $K$ is admissible $(d=2)$. Let $\boldsymbol{u}_{0} \in G_{\text {div }}, \varphi_{0} \in V \cap L^{\infty}(\Omega)$ with $F\left(\varphi_{0}\right) \in L^{1}(\Omega)$ and $M\left(\varphi_{0}\right) \in L^{1}(\Omega)$, where $M$ is defined as in Theorem 2.3. Let also $\boldsymbol{v} \in$ $L_{t b}^{2}\left(0, \infty ; G_{d i v}\right)$. Then there exists a weak solution $[\boldsymbol{u}, \varphi]$ to system $(1.2)-(1.6)$ such that

$$
\begin{aligned}
& \boldsymbol{u} \in L^{\infty}\left(0, \infty ; G_{d i v}\right) \cap L_{t b}^{2}\left(0, \infty ; V_{d i v}\right), \quad \boldsymbol{u}_{t} \in L_{t b}^{2}\left(0, \infty ; V_{d i v}^{\prime}\right), \\
& \varphi \in L^{\infty}(0, \infty ; V) \cap L_{t b}^{2}\left(0, \infty ; H^{2}(\Omega)\right), \quad \varphi_{t} \in L_{t b}^{2}(0, \infty ; H) .
\end{aligned}
$$

If, in addition, $\boldsymbol{u}_{0} \in V_{\text {div }}$ and $\varphi_{0} \in V \cap C^{\beta}(\bar{\Omega})$ for some $\beta \in(0,1)$, then the (unique) strong solution given by Theorem 3.6 satisfies (5.2) and

$$
\boldsymbol{u} \in L^{\infty}\left(0, \infty ; V_{d i v}\right) \cap L_{t b}^{2}\left(0, \infty ; H^{2}(\Omega)^{2}\right), \quad \boldsymbol{u}_{t} \in L_{t b}^{2}\left(0, \infty ; G_{d i v}\right) .
$$

Finally, suppose that $\varphi_{0} \in H^{2}(\Omega)$ satisfies (3.11). Then the strong solution also enjoys the following properties:

$$
\varphi \in L^{\infty}\left(0, \infty ; H^{2}(\Omega)\right), \quad \varphi_{t} \in L^{\infty}(0, \infty ; H) \cap L_{t b}^{2}(0, T ; V) .
$$

Moreover, there exists a constant $\Gamma=\Gamma(\kappa)$, depending on $\kappa \in[0,1]$, on $\|\boldsymbol{v}\|_{L_{t b}^{2}\left(0, \infty ; G_{d i v}\right)}$ (and on $F, m, K, \nu, \Omega)$, such that, for every initial data $\left[\boldsymbol{u}_{0}, \varphi_{0}\right] \in V_{\text {div }} \times H^{2}(\Omega)$, with $\varphi_{0}$ satisfying (3.11), $F\left(\varphi_{0}\right), M\left(\varphi_{0}\right) \in L^{1}(\Omega)$ (hence $\left|\varphi_{0}\right| \leq 1$ almost everywhere in $\Omega$ ), and $\left|\bar{\varphi}_{0}\right| \leq \kappa$, there exists a time $t_{1}=t_{1}\left(E\left(\boldsymbol{u}_{0}, \varphi_{0}\right)\right) \geq 0$, where $E\left(\boldsymbol{u}_{0}, \varphi_{0}\right)$ is given by (5.9), such that the strong solution corresponding to $\left[\boldsymbol{u}_{0}, \varphi_{0}\right]$ satisfies the dissipative estimate

$$
\|\boldsymbol{u}(t)\|_{V_{\text {div }}}^{2}+\int_{t}^{t+1}\|\boldsymbol{u}(s)\|_{H^{2}(\Omega)^{2}}^{2} d s+\|\varphi(t)\|_{H^{2}(\Omega)}^{2} \leq \Gamma(k), \quad \forall t \geq t_{1} .
$$

Proof. First we observe that, by arguing as in [24, Proof of Proposition 2], from (2.3) we can deduce the differential inequality

$$
\frac{d}{d t}\left(\|\boldsymbol{u}\|^{2}+\|\varphi\|^{2}\right)+\alpha_{0}\|\nabla \varphi\|^{2}+\nu_{1}\|\nabla \boldsymbol{u}\|^{2} \leq \hat{C}+\frac{1}{\nu_{1} \lambda_{1}}\|\boldsymbol{v}\|^{2} .
$$

Moreover, again by arguing as in [24, Proof of Proposition 2] (see also [14, Proof of Corollary 2]), we infer from (5.6) the dissipative estimate

$$
\|\boldsymbol{u}(t)\|^{2}+\|\varphi(t)\|^{2} \leq\left(\left\|\boldsymbol{u}_{0}\right\|^{2}+\left\|\varphi_{0}\right\|^{2}\right) e^{-\ell t}+L, \quad \forall t \geq 0,
$$

where the positive constant $L$ depends on $\bar{\varphi}_{0}$ and on $\|\boldsymbol{v}\|_{L_{t b}^{2}\left(0 ; \infty ; G_{d i v}\right)}$. This, in particular, entails that $\boldsymbol{u} \in L^{\infty}\left(0, \infty ; G_{d i v}\right)$. Let us now integrate (5.6) between $t$ and $t+1$. We get

$$
\begin{aligned}
& \|\boldsymbol{u}(t+1)\|^{2}+\|\varphi(t+1)\|^{2}+\alpha_{0} \int_{t}^{t+1}\|\nabla \varphi(s)\|^{2} d s+\nu_{1} \int_{t}^{t+1}\|\nabla \boldsymbol{u}(s)\|^{2} d s \\
& \leq\|\boldsymbol{u}(t)\|^{2}+\|\varphi(t)\|^{2}+\hat{C}+\frac{1}{\nu_{1} \lambda_{1}} \int_{t}^{t+1}\|\boldsymbol{v}(s)\|^{2} d s, \quad \forall t \geq 0 .
\end{aligned}
$$


Hence, (5.7) and (5.8) yield that

$$
\frac{\alpha_{0}}{2} \int_{t}^{t+1}\|\nabla \varphi(s)\|^{2} d s+\frac{\nu_{1}}{2} \int_{t}^{t+1}\|\nabla \boldsymbol{u}(s)\|^{2} d s \leq E\left(\boldsymbol{u}_{0}, \varphi_{0}\right) e^{-\ell t}+\Gamma_{0}, \quad \forall t \geq 0
$$

where we have set

$$
E\left(\boldsymbol{u}_{0}, \varphi_{0}\right):=\frac{1}{2}\left(\left\|\boldsymbol{u}_{0}\right\|^{2}+\left\|\varphi_{0}\right\|^{2}\right)
$$

and where $\Gamma_{0}=\mathbb{Q}\left(\kappa,\|\boldsymbol{v}\|_{L_{t b}^{2}\left(0, \infty ; G_{d i v}\right)}\right)$, with $\kappa \in[0,1]$ such that $\left|\bar{\varphi}_{0}\right| \leq \kappa$. In particular, this gives

$$
\boldsymbol{u} \in L_{t b}^{2}\left(\mathbb{R}_{+} ; V_{\text {div }}\right), \quad \varphi \in L_{t b}^{2}\left(\mathbb{R}_{+} ; V\right) .
$$

Moreover, there exists a time $t_{0}=t_{0}\left(E\left(\boldsymbol{u}_{0}, \varphi_{0}\right)\right)>0$, which can be given by $t_{0}=\frac{1}{\ell} \log E\left(\boldsymbol{u}_{0}, \varphi_{0}\right)$, such that

$$
\frac{\alpha_{0}}{2} \int_{t}^{t+1}\|\nabla \varphi(s)\|^{2} d s+\frac{\nu_{1}}{2} \int_{t}^{t+1}\|\nabla \boldsymbol{u}(s)\|^{2} d s \leq \Gamma_{0}+1, \quad \forall t \geq t_{0} .
$$

Let us now begin with the higher-order estimates. We test $(3.1)$ by $B(\varphi)_{t}=\lambda(\varphi) \varphi_{t}$. On account of (3.3), we obtain the identity

$$
\begin{aligned}
& \frac{1}{2} \frac{d}{d t}\|\nabla B(\varphi)\|^{2}+\int_{\Omega} \lambda(\varphi) \varphi_{t}^{2}+\left(\boldsymbol{u} \cdot \nabla \varphi, \lambda(\varphi) \varphi_{t}\right) \\
& =\left(m(\varphi)(\nabla K * \varphi), \nabla B(\varphi)_{t}\right) .
\end{aligned}
$$

Observe that

$$
\nabla B(\varphi)_{t}=\lambda(\varphi) \nabla \varphi_{t}+\lambda^{\prime}(\varphi) \nabla \varphi \varphi_{t}
$$

The term on the right-hand side of (5.11) can be written as follows:

$$
\begin{aligned}
& \left(m(\varphi)(\nabla K * \varphi), \nabla B(\varphi)_{t}\right) \\
& =\frac{d}{d t}(m(\varphi)(\nabla K * \varphi), \lambda(\varphi) \nabla \varphi)-\left(m^{\prime}(\varphi) \varphi_{t}(\nabla K * \varphi), \lambda(\varphi) \nabla \varphi\right) \\
& -\left(m(\varphi)\left(\nabla K * \varphi_{t}\right), \lambda(\varphi) \nabla \varphi\right) .
\end{aligned}
$$

Therefore, plugging (5.13) in the differential identity (5.11), we get

$$
\begin{aligned}
& \frac{1}{2} \frac{d \Phi}{d t}+\int_{\Omega} \lambda(\varphi) \varphi_{t}^{2}+\left(\boldsymbol{u} \cdot \nabla \varphi, \lambda(\varphi) \varphi_{t}\right) \\
& =-\left(m^{\prime}(\varphi) \varphi_{t}(\nabla K * \varphi), \lambda(\varphi) \nabla \varphi\right)-\left(m(\varphi)\left(\nabla K * \varphi_{t}\right), \lambda(\varphi) \nabla \varphi\right),
\end{aligned}
$$

where we have set

$$
\Phi:=\|\nabla B(\varphi)\|^{2}-2(m(\varphi)(\nabla K * \varphi), \lambda(\varphi) \nabla \varphi) .
$$

On account of assumptions (A1), (A4), which ensure that $\alpha_{0} \leq \lambda(s) \leq \lambda_{\infty}$, for all $s \in[-1,1]$, it is immediate to see that the right-hand side of (5.14) can be bounded from above by

$$
\frac{\alpha_{0}}{4}\left\|\varphi_{t}\right\|^{2}+C_{m, \lambda, K}\|\nabla \varphi\|^{2}+C_{m, K}
$$

As far as the advective term on the left-hand side of (5.14) is concerned, since $\nabla B(\varphi)=\lambda(\varphi) \nabla \varphi$, we have that

$$
\begin{aligned}
\left|\left(\boldsymbol{u} \cdot \nabla \varphi, \lambda(\varphi) \varphi_{t}\right)\right| & =\left|\left(\boldsymbol{u} \cdot \nabla B(\varphi), \varphi_{t}\right)\right| \\
& \leq\|\boldsymbol{u}\|_{L^{4}(\Omega)^{2}}\|\nabla B(\varphi)\|_{L^{4}(\Omega)^{2}}\left\|\varphi_{t}\right\| \\
& \leq C\|\boldsymbol{u}\|^{1 / 2}\|\nabla \boldsymbol{u}\|^{1 / 2}\|\nabla B(\varphi)\|^{1 / 2}\|B(\varphi)\|_{H^{2}(\Omega)}^{1 / 2}\left\|\varphi_{t}\right\| .
\end{aligned}
$$


Let us now control the $H^{2}$-norm of $B(\varphi)$ in terms of the $L^{2}$-norm of $\varphi_{t}$. To this end, we first employ elliptic regularity, namely

$$
\|B(\varphi)\|_{H^{2}(\Omega)} \leq C\left(\|\Delta B(\varphi)\|+\|B(\varphi)\|_{V}+\|\nabla B(\varphi) \cdot \boldsymbol{n}\|_{H^{1 / 2}(\partial \Omega)}\right) .
$$

Then we estimate the boundary term on the right-hand side by taking (3.3) into account. Arguing in a similar way as in the time discrete version (4.25), we find that

$$
\begin{aligned}
& \|\nabla B(\varphi) \cdot \boldsymbol{n}\|_{H^{1 / 2}(\partial \Omega)}=\|m(\varphi)(\nabla K * \varphi) \cdot \boldsymbol{n}\|_{H^{1 / 2}(\partial \Omega)} \\
& \leq\|m(\varphi)\|_{L^{\infty}(\partial \Omega)}\|(\nabla K * \varphi) \cdot \boldsymbol{n}\|_{H^{1 / 2}(\partial \Omega)}+\|(\nabla K * \varphi) \cdot \boldsymbol{n}\|_{L^{\infty}(\partial \Omega)}\|m(\varphi)\|_{H^{1 / 2}(\partial \Omega)} \\
& \leq m_{\infty} C_{\Omega}\|K * \varphi\|_{H^{2}(\Omega)}+C_{K, \Omega} m_{\infty}^{\prime}\|\varphi\|_{H^{1 / 2}(\partial \Omega)}+C_{K, \Omega} m_{\infty}|\partial \Omega|_{1}^{1 / 2} \\
& \leq C_{m, K, \Omega}(\|\nabla B(\varphi)\|+1),
\end{aligned}
$$

thanks to the fact that $|\varphi| \leq 1$ almost everywhere in $Q_{T}$. Therefore, on account of (3.1) and (5.17), from (5.16) we obtain that

$$
\begin{aligned}
\|B(\varphi)\|_{H^{2}(\Omega)} & \leq C(\|\Delta B(\varphi)\|+\|\nabla B(\varphi)\|+1) \\
& \leq C\left(\left\|\varphi_{t}\right\|+\|\boldsymbol{u} \cdot \nabla \varphi\|+\|\operatorname{div}(m(\varphi)(\nabla K * \varphi))\|+\|\nabla B(\varphi)\|+1\right) \\
& \leq C\left(\left\|\varphi_{t}\right\|+\left\|\boldsymbol{u} \cdot \frac{1}{\lambda} \nabla B(\varphi)\right\|+\|\nabla B(\varphi)\|+1\right) \\
& \leq C\left(\left\|\varphi_{t}\right\|+\|\boldsymbol{u}\|_{L^{4}(\Omega)^{2}}\|\nabla B(\varphi)\|_{L^{4}(\Omega)^{2}}+\|\nabla B(\varphi)\|+1\right) \\
& \leq C\left(\left\|\varphi_{t}\right\|+\|\boldsymbol{u}\|^{1 / 2}\|\nabla \boldsymbol{u}\|^{1 / 2}\|\nabla B(\varphi)\|^{1 / 2}\|B(\varphi)\|_{H^{2}(\Omega)}^{1 / 2}+\|\nabla B(\varphi)\|+1\right) .
\end{aligned}
$$

Thanks to Young's inequality, (5.18) entails the desired estimate

$$
\|B(\varphi)\|_{H^{2}(\Omega)} \leq C\left(\left\|\varphi_{t}\right\|+\|\boldsymbol{u}\|\|\nabla \boldsymbol{u}\|\|\nabla B(\varphi)\|+\|\nabla B(\varphi)\|+1\right) .
$$

Estimating the term in the $H^{2}$-norm of $B$ in (5.15) by means of (5.19), we get

$$
\left|\left(\boldsymbol{u} \cdot \nabla \varphi, \lambda(\varphi) \varphi_{t}\right)\right| \leq \frac{\alpha_{0}}{4}\left\|\varphi_{t}\right\|^{2}+C\left(\|\boldsymbol{u}\|^{2}\|\nabla \boldsymbol{u}\|^{2}\|\nabla B(\varphi)\|^{2}+\|\nabla B(\varphi)\|^{2}+1\right) .
$$

Therefore, estimating the advective term in (5.14) through (5.20), the other terms as done above, we are led to the differential inequality

$$
\frac{d \Phi}{d t}+\alpha_{0}\left\|\varphi_{t}\right\|^{2} \leq C_{m, \lambda, K}\left(1+\|\boldsymbol{u}\|^{2}\|\nabla \boldsymbol{u}\|^{2}\right)\left(1+\|\nabla \varphi\|^{2}\right) .
$$

On the other hand, it is easy to see that there are two constants $K_{1}, K_{2}>0$, depending on $m, \lambda$ and $K$, such that

$$
K_{1}\left(\|\nabla \varphi(t)\|^{2}-1\right) \leq \Phi(t) \leq K_{2}\left(\|\nabla \varphi(t)\|^{2}+1\right) .
$$

Therefore, on account of (5.10) and the fact that $\boldsymbol{u} \in L^{\infty}\left(0, \infty ; G_{d i v}\right)$, by applying the uniform Gronwall's Lemma, from (5.21) and (5.22), we can find a time $t_{1}=t_{0}+1$ such that

$$
\|\varphi(t)\|_{V}^{2} \leq \Gamma_{1}(\kappa), \quad \forall t \geq t_{1} .
$$

Moreover, by integrating (5.21) between $t$ and $t+1$, for all $t \geq t_{1}$, we also get that

$$
\alpha_{0} \int_{t}^{t+1}\left\|\varphi_{t}(s)\right\|^{2} d s \leq \Gamma_{2}(\kappa), \quad \forall t \geq t_{1} .
$$

In summary, we have shown that

$$
\varphi \in L^{\infty}\left(\mathbb{R}_{+} ; V\right), \quad \varphi_{t} \in L_{t b}^{2}\left(\mathbb{R}_{+} ; H\right) .
$$


We now prove that $\varphi \in L_{t b}^{2}\left(\mathbb{R}_{+} ; H^{2}(\Omega)\right)$. First, from (5.10), (5.24), (5.23), and (5.19), we infer that

$$
\int_{t}^{t+1}\|B(\varphi(s))\|_{H^{2}(\Omega)}^{2} d s \leq \Gamma_{3}(\kappa), \quad \forall t \geq t_{1},
$$

whence $B(\varphi) \in L_{t b}^{2}\left(\mathbb{R}_{+} ; H^{2}(\Omega)\right)$. This bound, together with the Gagliardo-Nirenberg inequality (2.1) and (5.23), implies that (cf. (4.33))

$$
\begin{aligned}
& \int_{t}^{t+1}\|\nabla \varphi(s)\|_{L^{p}(\Omega)^{2}}^{2 p /(p-2)} d s+\int_{t}^{t+1}\|\nabla B(\varphi(s))\|_{L^{p}(\Omega)^{2}}^{2 p /(p-2)} d s+\int_{t}^{t+1}\|\nabla \lambda(\varphi(s))\|_{L^{p}(\Omega)^{2}}^{2 p /(p-2)} d s \\
& \leq \Gamma_{4}(\kappa),
\end{aligned}
$$

for all $t \geq t_{1}$ and $2<p<\infty$. Thus, we have

$$
\varphi, B(\varphi), \lambda(\varphi) \in L_{t b}^{2 p /(p-2)}\left(\mathbb{R}_{+} ; W^{1, p}(\Omega)\right) .
$$

Notice that we have used the identity $\nabla \lambda(\varphi)=\lambda^{\prime}(\varphi) \nabla \varphi$. As far as the second spatial derivatives $\partial_{i j}^{2} \varphi$ are concerned, recall that we have the identity (cf. (4.13))

$$
\partial_{i j}^{2} \varphi=\frac{1}{\lambda(\varphi)} \partial_{i j}^{2} B(\varphi)-\frac{1}{\lambda^{2}(\varphi)} \partial_{i} \lambda(\varphi) \partial_{j} B(\varphi)
$$

Combining this with (5.26) and (5.27) (when $p=4$ ), we obtain that

$$
\int_{t}^{t+1}\|\varphi(s)\|_{H^{2}(\Omega)}^{2} d s \leq \Gamma_{5}(\kappa), \quad \forall t \geq t_{1}
$$

so that $\varphi \in L_{t b}^{2}\left(\mathbb{R}_{+} ; H^{2}(\Omega)\right)$. This concludes the proof of the first part of the theorem.

Let us now assume that $\boldsymbol{u}_{0} \in V_{\text {div }}$ and that $\varphi_{0} \in V \cap C^{\beta}(\bar{\Omega})$. On account of (5.10), assumption (V), (5.24) and (5.27) (when $p=4$ ), the application of the uniform Gronwall's Lemma to (4.66) gives

$$
\|\boldsymbol{u}(t)\|_{V_{\text {div }}} \leq \Gamma_{6}(\kappa), \quad \forall t \geq t_{1},
$$

namely, $\boldsymbol{u} \in L^{\infty}\left(\mathbb{R}_{+} ; V_{\text {div }}\right)$. By integrating (4.66) between $t$ and $t+1$, and using Proposition 4.1, (4.57)-(4.58), it is not difficult to conclude that

$$
\int_{t}^{t+1}\left\|\boldsymbol{u}_{t}(s)\right\|^{2} d s+\int_{t}^{t+1}\|\boldsymbol{u}(s)\|_{H^{2}(\Omega)^{2}}^{2} d s \leq \Gamma_{7}(\kappa), \quad \forall t \geq t_{1} .
$$

Thus, we infer that

$$
\boldsymbol{u}_{t} \in L_{t b}^{2}\left(\mathbb{R}_{+} ; G_{d i v}\right) \text { and } \boldsymbol{u} \in L_{t b}^{2}\left(\mathbb{R}_{+} ; H^{2}(\Omega)^{2}\right)
$$

In order to prove (5.4), we take the time derivative of (3.1) and test the resulting equation by $\varphi_{t}$. By using the boundary condition (3.3), we obtain the following identity:

$$
\begin{aligned}
& \frac{1}{2} \frac{d}{d t}\left\|\varphi_{t}\right\|^{2}+\left(\nabla B(\varphi)_{t}, \nabla \varphi_{t}\right) \\
& =-\left(\boldsymbol{u}_{t} \cdot \nabla \varphi, \varphi_{t}\right)+\left(m^{\prime}(\varphi) \varphi_{t}(\nabla K * \varphi), \nabla \varphi_{t}\right)+\left(m(\varphi)\left(\nabla K * \varphi_{t}\right), \nabla \varphi_{t}\right) .
\end{aligned}
$$

Owing to the fact that $\nabla B(\varphi)=\lambda(\varphi) \nabla \varphi$, and recalling (4.5), we find that

$$
\begin{aligned}
& \left(\nabla B(\varphi)_{t}, \nabla \varphi_{t}\right) \geq \alpha_{0}\left\|\nabla \varphi_{t}\right\|^{2}+\left(\lambda^{\prime}(\varphi) \nabla \varphi \varphi_{t}, \nabla \varphi_{t}\right) \\
& \geq \frac{\alpha_{0}}{2}\left\|\nabla \varphi_{t}\right\|^{2}-\frac{\lambda_{\infty}^{\prime 2}}{2 \alpha_{0}}\left\|\varphi_{t} \nabla \varphi\right\|^{2} .
\end{aligned}
$$


As far as the last term in (5.32) is concerned, we have

$$
\begin{aligned}
& \left\|\varphi_{t} \nabla \varphi\right\|^{2} \leq\left\|\varphi_{t}\right\|_{L^{4}(\Omega)}^{2}\|\nabla \varphi\|_{L^{4}(\Omega)^{2}}^{2} \\
& \leq C\left(\left\|\varphi_{t}\right\|^{2}+\left\|\varphi_{t}\right\|\left\|\nabla \varphi_{t}\right\|\right)\left\|\lambda^{-1} \nabla B(\varphi)\right\|_{L^{4}(\Omega)^{2}}^{2} \\
& \leq C\left(\left\|\varphi_{t}\right\|^{2}+\left\|\varphi_{t}\right\|\left\|\nabla \varphi_{t}\right\|\right)\|\nabla B(\varphi)\|\|B(\varphi)\|_{H^{2}(\Omega)} \\
& \leq \delta\left\|\nabla \varphi_{t}\right\|^{2}+C_{\delta}\left\|\varphi_{t}\right\|^{2}\|B(\varphi)\|_{H^{2}(\Omega)}^{2}+C\left\|\varphi_{t}\right\|^{2}
\end{aligned}
$$

for all $\delta>0$, where the first of (5.25) has been taken into account, as well as the fact that $B(\varphi) \in L^{\infty}(0, \infty ; V)$. Hence, combining this estimate with (5.32) and choosing $\delta>0$ small enough, we obtain the bound

$$
\left(\nabla B(\varphi)_{t}, \nabla \varphi_{t}\right) \geq \frac{\alpha_{0}}{4}\left\|\nabla \varphi_{t}\right\|^{2}-C\left\|\varphi_{t}\right\|^{2}\|B(\varphi)\|_{H^{2}(\Omega)}^{2}-C\left\|\varphi_{t}\right\|^{2} .
$$

The $H^{2}$-norm of $B(\varphi)$ can be expressed in terms of the $L^{2}$-norm of $\varphi_{t}$ by arguing as above (cf. (5.16)-(5.18)), i.e., by first using elliptic regularity theory and then by estimating the boundary term, to get (5.19). From (5.19), on account of the improved regularity of the first of (5.25) and (5.29), we get that

$$
\|B(\varphi)\|_{H^{2}(\Omega)} \leq C\left(\left\|\varphi_{t}\right\|+1\right) .
$$

Let us now estimate the terms on the right-hand side of (5.31). For the first term, on account of the first regularity of (5.25) and (5.34), we have

$$
\begin{aligned}
& \left|-\left(\boldsymbol{u}_{t} \cdot \nabla \varphi, \varphi_{t}\right)\right| \\
& \leq C\left\|\boldsymbol{u}_{t}\right\|\left\|\varphi_{t}\right\|_{L^{4}(\Omega)}\left\|\frac{1}{\lambda(\varphi)} \nabla B(\varphi)\right\|_{L^{4}(\Omega)^{2}} \\
& \leq C\left\|\boldsymbol{u}_{t}\right\|\left(\left\|\varphi_{t}\right\|+\left\|\varphi_{t}\right\|^{1 / 2}\left\|\nabla \varphi_{t}\right\|^{1 / 2}\right)\|\nabla B(\varphi)\|^{1 / 2}\|B(\varphi)\|_{H^{2}(\Omega)}^{1 / 2} \\
& \leq C\left\|\boldsymbol{u}_{t}\right\|\left(\left\|\varphi_{t}\right\|+\left\|\varphi_{t}\right\|^{1 / 2}\left\|\nabla \varphi_{t}\right\|^{1 / 2}\right)\left(\left\|\varphi_{t}\right\|^{1 / 2}+1\right) \\
& \leq 3 \delta\left\|\nabla \varphi_{t}\right\|^{2}+C_{\delta}\left(\left\|\varphi_{t}\right\|^{4}+\left\|\boldsymbol{u}_{t}\right\|^{2}+1\right) .
\end{aligned}
$$

As far as the remaining terms on the right-hand side of (5.31) are concerned, they can simply be controlled by

$$
\delta\left\|\nabla \varphi_{t}\right\|^{2}+C_{\delta}\left\|\varphi_{t}\right\|^{2} .
$$

Therefore, collecting (5.33)-(5.36) into (5.31), we deduce the differential inequality

$$
\frac{d}{d t}\left\|\varphi_{t}\right\|^{2}+\frac{\alpha_{0}}{4}\left\|\nabla \varphi_{t}\right\|^{2} \leq C\left(\left\|\varphi_{t}\right\|^{4}+\left\|\varphi_{t}\right\|^{2}+\left\|\boldsymbol{u}_{t}\right\|^{2}+1\right)
$$

Then, using (5.24), (5.30) and the uniform Gronwall's Lemma, we obtain that

$$
\left\|\varphi_{t}(t)\right\|^{2} \leq \Gamma_{8}(\kappa), \quad \forall t \geq t_{1}
$$

whence we have $\varphi_{t} \in L^{\infty}\left(\mathbb{R}_{+} ; H\right)$. By integrating (5.37) between $t$ and $t+1$, for $t \geq t_{1}$, we also get

$$
\int_{t}^{t+1}\left\|\nabla \varphi_{t}(s)\right\|^{2} d s \leq \Gamma_{9}(\kappa), \quad \forall t \geq t_{1}
$$

so that $\varphi_{t} \in L_{t b}^{2}\left(\mathbb{R}_{+} ; V\right)$. Finally, we prove that $\varphi \in L^{\infty}\left(\mathbb{R}_{+} ; H^{2}(\Omega)\right)$. First, notice that (5.34) and (5.38) entail that $\|B(\varphi(t))\|_{H^{2}(\Omega)} \leq \Gamma_{10}(\kappa)$, for all $t \geq t_{1}$. Then we have

$$
\|\varphi(t)\|_{W^{1, p}(\Omega)}+\|B(\varphi(t))\|_{W^{1, p}(\Omega)}+\|\lambda(\varphi(t))\|_{W^{1, p}(\Omega)} \leq \Gamma_{11}(\kappa), \quad \forall t \geq t_{1},
$$


for any $p \in(2, \infty)$, whence

$$
\varphi, B(\varphi), \lambda(\varphi) \in L^{\infty}\left(\mathbb{R}_{+} ; W^{1, p}(\Omega)\right) .
$$

Therefore, recalling (5.28) and employing (5.39), we deduce that

$$
\|\varphi(t)\|_{H^{2}(\Omega)} \leq \Gamma_{12}(\kappa), \quad \forall t \geq t_{1}
$$

which is the final desired claim. The proof is complete.

Remark 5.2. Assume that $\boldsymbol{u}_{0} \in V_{d i v}, \varphi_{0} \in H^{2}(\Omega)$, and that the compatibility condition (3.11) is satisfied. Moreover, assume also that

(M1b): The mobility satisfies (M) and also $m \in C^{2}[-1,1]$.

(A1t): $F \in C^{4}(-1,1)$ and $\lambda:=m F^{\prime \prime} \in C^{2}[-1,1]$.

Then the following time continuity properties for the strong solution of Theorem 3.6 hold true:

$$
\boldsymbol{u} \in C^{0}\left([0, T] ; V_{\text {div }}\right), \quad \varphi \in C^{0}\left([0, T] ; H^{2}(\Omega)\right) \cap C^{1}([0, T] ; H) .
$$

Let us sketch the argument for proving (5.40), omitting some details. The time continuity of the velocity field (see the first of (5.40)) is a consequence of the fact that $\boldsymbol{u} \in C_{w}\left([0, T] ; V_{\text {div }}\right)$ and of the differential identity

$$
\begin{aligned}
& \frac{1}{2} \frac{d}{d t}\|\nabla \boldsymbol{u}\|^{2}-(\nu(\varphi) \Delta \boldsymbol{u}, S \boldsymbol{u})-2\left(\nu^{\prime}(\varphi) \nabla \varphi \cdot D \boldsymbol{u}, S \boldsymbol{u}\right)+(\mathcal{B} \boldsymbol{u}, S \boldsymbol{u}) \\
& =((-K * \varphi) \nabla \varphi, S \boldsymbol{u})+(\boldsymbol{v}, S \boldsymbol{u}),
\end{aligned}
$$

which is deduced by testing (4.57) and (4.58) by $S \boldsymbol{u}$ (recall that $S:=-P \Delta$ is the Stokes operator, cf. Section 2). In order to show the second of (5.40), we first observe that from (5.31), and from the regularity properties $(3.10)$, (3.9), it is not difficult to see that $\left\|\varphi_{t}(\cdot)\right\|^{2} \in C^{0}[0, T]$. Moreover, (3.12) implies that $\varphi \in C^{0}([0, T] ; V)$. From this, we infer that $B(\varphi) \in C^{0}([0, T] ; V)$. Since $\varphi, B(\varphi) \in L^{\infty}\left(0, T ; H^{2}(\Omega)\right)$, we then have $\varphi, B(\varphi) \in C_{w}\left([0, T] ; H^{2}(\Omega)\right)$. Also, recalling that $\boldsymbol{u} \in$ $C^{0}\left([0, T] ; L^{4}(\Omega)\right)$ and $\nabla \varphi \in C_{w}\left([0, T] ; L^{4}(\Omega)\right)$, we have that $\boldsymbol{u} \cdot \nabla \varphi \in C_{w}([0, T] ; H)$. It is also easy to see that $\operatorname{div}(m(\varphi)(\nabla K * \varphi)) \in C^{0}([0, T] ; H)$. Hence, (3.1) yields $\varphi_{t} \in C_{w}([0, T] ; H)$. This weak in time continuity, together with the $L^{2}$-norm continuity for $\varphi_{t}$, implies that $\varphi_{t} \in C^{0}([0, T] ; H)$. On the other hand, we also have $\varphi \in C^{0}\left([0, T] ; H^{s}(\Omega)\right)$, for $1 \leq s<2$, whence $\nabla \varphi \in C^{0}\left([0, T] ; L^{4}(\Omega)\right)$. Therefore, $\boldsymbol{u} \cdot \nabla \varphi \in C^{0}([0, T] ; H)$, and, from (3.1) once again, we infer that $\Delta B(\varphi) \in C^{0}([0, T] ; H)$. We now employ the estimate

$$
\begin{aligned}
& \left\|\varphi_{2}-\varphi_{1}\right\|_{H^{2}(\Omega)}+\left\|B\left(\varphi_{2}\right)-B\left(\varphi_{1}\right)\right\|_{H^{2}(\Omega)} \\
& \leq C\left\|\Delta\left(B\left(\varphi_{2}\right)-B\left(\varphi_{1}\right)\right)\right\|+C\left\|\varphi_{1}-\varphi_{2}\right\|_{V},
\end{aligned}
$$

which requires slightly stronger assumptions than $(\mathbf{M})$ and (A1), that is, (M1b) and (A1t) above. By means of (5.41), we eventually get that $\varphi, B(\varphi) \in C^{0}\left([0, T] ; H^{2}(\Omega)\right)$.

Let us now assume that the forcing function $\boldsymbol{v}$ is time independent, i.e., $\boldsymbol{v} \in G_{\text {div }}$. Following [24, Section 5], for $\kappa \in[0,1]$ fixed, we introduce the metric space $\mathcal{X}_{\kappa}$ defined by

$$
\mathcal{X}_{\kappa}:=G_{d i v} \times \mathcal{Y}_{\kappa},
$$

with $\mathcal{Y}_{\kappa}$ given by

$$
\mathcal{Y}_{\kappa}:=\left\{\varphi \in L^{\infty}(\Omega):|\varphi| \leq 1 \text { a.e. in } \Omega, F(\varphi), M(\varphi) \in L^{1}(\Omega),|\bar{\varphi}| \leq \kappa\right\} .
$$

The metric on $\mathcal{X}_{\kappa}$ is

$$
\boldsymbol{d}_{\mathcal{X}_{\kappa}}\left(\boldsymbol{z}_{2}, \boldsymbol{z}_{1}\right):=\left\|\boldsymbol{u}_{2}-\boldsymbol{u}_{1}\right\|+\left\|\varphi_{2}-\varphi_{1}\right\|
$$

for every $\boldsymbol{z}_{1}:=\left[\boldsymbol{u}_{1}, \varphi_{1}\right]$ and $\boldsymbol{z}_{2}:=\left[\boldsymbol{u}_{2}, \varphi_{2}\right]$ in $\mathcal{X}_{\kappa}$. 
Suppose that $(\mathbf{K}),(\mathbf{V}),(\mathbf{M}),(\mathbf{A} \mathbf{1})-(\mathbf{A} 4)$ are satisfied. Then we know that the set $\mathcal{G}_{\kappa}$ of all weak solutions to (1.1)-(1.6) from $[0, \infty)$ to $\mathcal{X}_{k}$ (cf. Definition 2.2 and Theorem 2.3), corresponding to all initial data $\boldsymbol{z}_{0}=\left[\boldsymbol{u}_{0}, \varphi_{0}\right] \in \mathcal{X}_{\kappa}$, is a generalized semiflow on $\mathcal{X}_{\kappa}$ (in the sense of [6]), which possesses a (unique) global attractor $\mathcal{A}_{\kappa}$ (see [24, Section 5]). Notice that in [24, Section 5] the viscosity $\nu$ was assumed to be constant, for simplicity. However, the arguments therein can easily be adapted also to the case of nonconstant viscosity satisfying (V). We also remark that uniqueness of weak solutions is not known, in general. However, if $\nu$ is constant, then, thanks to the uniqueness result of [20, Theorem 4] (cf. (2.5)), the generalized semiflow becomes a semigroup of closed operators on $\mathcal{X}_{\kappa}$, and the global attractor is connected.

Assume now that the assumptions of Proposition 5.1 are satisfied. Take $\boldsymbol{z}_{0} \in \mathcal{X}_{\kappa}$ and consider a weak solution $\boldsymbol{z}:=[\boldsymbol{u}, \varphi] \in C^{0}\left([0, \infty) ; \mathcal{X}_{\kappa}\right)$ corresponding to $\boldsymbol{z}_{0}$. By integrating (5.6) in time between 0 and $\tau>0$, we can deduce that, for every $\tau>0$, there exists some $t_{\tau} \in(0, \tau]$ such that $\boldsymbol{z}\left(t_{\tau}\right) \in V_{\text {div }} \times V$. We now consider $(5.21)$ in $\left[t_{\tau}, \infty\right)$. By integrating this differential inequality between $t_{\tau}$ and $t>t_{\tau}$, we can see that there exists some $s_{\tau} \in\left(t_{\tau}, t\right]$ such that $\varphi_{t}\left(s_{\tau}\right) \in H$. This, assuming also that $\boldsymbol{u}\left(s_{\tau}\right) \in V_{\text {div }}$ and $\varphi\left(s_{\tau}\right) \in V$, owing to (5.19) and (5.28), implies that $\varphi\left(s_{\tau}\right) \in$ $H^{2}(\Omega)$. Moreover, since the boundary condition (3.3) holds almost everywhere on $\partial \Omega \times(0, T)$, we can suppose that $(3.11)$ holds in $s_{\tau}$ (i.e., with $\varphi_{0}$ replaced by $\varphi\left(s_{\tau}\right)$ ). Therefore, we can apply the last statement of Theorem 3.6 with initial time $s_{\tau}$. Let us then consider the metric space

$$
\mathcal{W}_{\kappa}:=V_{\text {div }} \times \mathcal{Z}_{k},
$$

where

$$
\begin{gathered}
\mathcal{Z}_{\kappa}:=\left\{\varphi \in H^{2}(\Omega): \nabla B(\varphi) \cdot \boldsymbol{n}=m(\varphi)(\nabla K * \varphi) \cdot \boldsymbol{n}, \text { a.e. on } \partial \Omega,\right. \\
\left.|\varphi| \leq 1 \text { a.e. in } \Omega, F(\varphi), M(\varphi) \in L^{1}(\Omega),|\bar{\varphi}| \leq \kappa\right\},
\end{gathered}
$$

endowed with the metric

$$
\boldsymbol{d}_{\mathcal{W}_{\kappa}}\left(\boldsymbol{z}_{2}, \boldsymbol{z}_{1}\right):=\left\|\boldsymbol{u}_{2}-\boldsymbol{u}_{1}\right\|_{V_{\text {div }}}+\left\|\varphi_{2}-\varphi_{1}\right\|_{H^{2}(\Omega)}, \quad \boldsymbol{z}_{1}, \boldsymbol{z}_{2} \in \mathcal{W}_{\kappa}
$$

Then, for every $\tau>0$, there exists $s_{\tau} \in(0, \tau]$ such that $\boldsymbol{z}\left(s_{\tau}\right) \in \mathcal{W}_{\kappa}$, and starting from the time $s_{\tau}$, the weak solution corresponding to $\boldsymbol{z}_{0}$ becomes a (unique) strong solution $\boldsymbol{z} \in C^{0}\left(\left[s_{\tau}, \infty\right) ; \mathcal{W}_{\kappa}\right)$ (cf. Remark 5.2). Furthermore, from $s_{\tau}$ on, this solution satisfies the dissipative estimate (5.5), namely, there exists a time $\widetilde{t}_{1}=\widetilde{t}_{1}\left(E\left(\boldsymbol{z}_{0}\right)\right) \geq s_{\tau}$ such that $\boldsymbol{z}$ satisfies (5.5) for all $t \geq \widetilde{t}_{1}$.

Let us now consider a subset $\mathcal{B} \subset \mathcal{X}_{k}$, bounded in the metric of $\mathcal{X}_{k}$. We can choose $\tau=1$ for every $\boldsymbol{z}_{0} \in \mathcal{B}$, and then infer that every weak solution starting from $\boldsymbol{z}_{0}$ becomes (at some time $s_{1} \in(0,1]$, which depends on $\boldsymbol{z}_{0}$ and on the weak solution considered from $\left.\boldsymbol{z}_{0}\right)$ a strong solution satisfying (5.5) for all $t \geq t_{1}^{*}$, with $t_{1}^{*}=t_{1}^{*}(R) \geq 1$, where $R>0$ is such that $\boldsymbol{d}_{\mathcal{X}_{\kappa}}(\boldsymbol{w}, \mathbf{0}) \leq R$, for all $\boldsymbol{w} \in \mathcal{B}$. Therefore, we deduce that there exists a time $t_{1}^{*}(\mathcal{B}) \geq 1$ such that

$$
\boldsymbol{z}(t) \in \mathcal{B}_{\mathcal{W}_{\kappa}}(\Lambda(k)), \quad \forall t \geq t_{1}^{*}
$$

where $\Lambda(k):=\Gamma^{1 / 2}(\kappa)$, and where $\mathcal{B}_{\mathcal{W}_{\kappa}}(\Lambda(k))$ is the closed ball in $\mathcal{W}_{\kappa}$ given by

$$
\mathcal{B}_{\mathcal{W}_{\kappa}}(\Lambda(k)):=\left\{\boldsymbol{w} \in \mathcal{W}_{\kappa}: \boldsymbol{d}_{\mathcal{W}_{\kappa}}(\boldsymbol{w}, \mathbf{0}) \leq \Lambda(k)\right\}
$$

Thanks to the full invariance property of the global attractor $\mathcal{A}_{\kappa}$, we immediately deduce that $\mathcal{A}_{\kappa} \subset \mathcal{B}_{\mathcal{W}_{\kappa}}(\Lambda(k))$. In conclusion, we have proven the following regularity result for the global attractor.

Theorem 5.3. Let $(\boldsymbol{K}),(\boldsymbol{V}),(\boldsymbol{M}),(\boldsymbol{A} \mathbf{1 b}),(\boldsymbol{A} 4)$ be satisfied, assume that $K \in W_{\text {loc }}^{2,1}\left(\mathbb{R}^{2}\right)$ or that $K$ is admissible $(d=2)$, and that $\boldsymbol{v} \in G_{\text {div }}$ is independent of time. Then the global attractor $\mathcal{A}_{k}$ of 
the generalized semiflow $\mathcal{G}_{k}$ associated with the nonlocal two-phase fluid system (1.1)-(1.6) is such that

$$
\mathcal{A}_{\kappa} \subset \mathcal{B}_{\mathcal{W}_{\kappa}}(\Lambda(k))
$$

Remark 5.4 (Corrigendum for [23]). Similarly to (3.11) of Theorem 3.6, also in [23, Theorem 2 and Proposition 1] a compatibility condition, associated with the assumption $\varphi_{0} \in H^{2}(\Omega)$, must be required. More precisely, setting $\mu_{0}:=a \varphi_{0}-J * \varphi_{0}+F^{\prime}\left(\varphi_{0}\right)$ (in [23], $J$ stands for the convolution kernel), the missing condition is $\nabla \mu_{0} \cdot \boldsymbol{n}=0$ almost everywhere on $\partial \Omega$. Consequently, the metric space $\mathcal{Y}_{m}^{1}$, for $m \geq 0$ fixed, introduced before the result on existence of the global attractor (see [23, Theorem 3]) must be defined as follows:

$$
\mathcal{Y}_{m}^{1}:=\left\{\varphi \in H^{2}(\Omega): \nabla \mu \cdot \boldsymbol{n}=0 \text { a.e. on } \partial \Omega, \mu=\varphi-J * \varphi+F^{\prime}(\varphi),|(\varphi, 1)| \leq m\right\} .
$$

This observation also applies to [20, Theorem 5], to the definition of the space $\mathcal{K}_{\eta}$ in [20, Theorem $10]$ ), and to [25, Theorem 2.3].

\section{The convective nonlocal CH Equation}

The results of the previous sections can essentially be established for the nonlocal Cahn-Hilliard equation with degenerate mobility and with a prescribed (divergent-free) velocity field $\boldsymbol{u}$. We shall consider both the cases $d=2,3$. However, when it comes to the regularity properties for $\varphi$ in dimension $d=3$, the results are not as strong as in the case $d=2$ (cf. Remark 6.3).

Theorem 6.1. Suppose that assumptions $(\boldsymbol{K}),(\boldsymbol{M}),(\boldsymbol{A} \mathbf{1 b}),(\boldsymbol{A} 4)$ are satisfied, and suppose that $K \in W_{\text {loc }}^{2,1}\left(\mathbb{R}^{2}\right)$ or that $K$ is admissible. Let $\varphi_{0} \in V \cap L^{\infty}(\Omega)$ with $F\left(\varphi_{0}\right) \in L^{1}(\Omega)$ and $M\left(\varphi_{0}\right) \in L^{1}(\Omega)$, where $M$ is defined as in Theorem 2.3. Assume also that $\boldsymbol{u}$ is given and

$$
\boldsymbol{u} \in L^{2 r /(r-d)}\left(0, T ; L_{d i v}^{r}(\Omega)^{d}\right), \quad d<r \leq \infty .
$$

Then, for every $T>0$, problem (1.2), (1.3), (1.5) $)_{2},(1.6)_{2}$ admits a strong solution $\varphi$ on $[0, T]$ such that

$$
\begin{aligned}
& \varphi \in L^{\infty}(0, T ; V) \cap H^{1}(0, T ; H), \\
& \varphi \in L^{2}\left(0, T ; H^{2}(\Omega)\right) .
\end{aligned}
$$

This solution is also unique provided that $r=\infty$ when $d=3$. If $d=2$ and $\boldsymbol{u}$ satisfies the additional regularity

$$
\boldsymbol{u} \in L^{s}\left(0, T ; L^{\infty}(\Omega)^{2}\right) \cap L^{\infty}\left(0, T ; L^{\sigma}(\Omega)^{2}\right), \quad s, \sigma>2, \quad \boldsymbol{u}_{t} \in L^{2}\left(0, T ; G_{\text {div }}\right),
$$

and $\varphi_{0} \in H^{2}(\Omega)$ satisfies (3.11), then the (unique) strong solution also satisfies

$$
\varphi \in L^{\infty}\left(0, T ; H^{2}(\Omega)\right), \quad \varphi_{t} \in L^{\infty}(0, T ; H) \cap L^{2}(0, T ; V) .
$$

Proof. Part (a). Since the argument follows the same lines of the time-discretization scheme of Step 1 and Step 3 in the proof of Theorem 3.6, we only briefly highlight the main points. The approximate problem (4.1)-(4.2) is considered, and, by applying time-discretization, we are led to formulate the incremental-step problem (4.9)-(4.10).

In view of (6.1), the bootstrap argument to prove that, for $\varphi_{0} \in V$, the solution to this problem satisfies $\left(\varphi_{1}, \ldots, \varphi_{N}\right) \in H^{2}(\Omega)^{N}$, is now a bit more delicate. Let us sketch this argument only for the case $d=3$. By comparison in (4.9)-(4.10), we first see that we have $\Delta B\left(\varphi_{k+1}\right) \in L^{p_{1}}(\Omega)$, where $p_{1}=2 r /(r+2)$, and $\nabla B\left(\varphi_{k+1}\right) \cdot \boldsymbol{n} \in H^{1 / 2}(\partial \Omega)$. By elliptic regularity theory, we then infer that $B\left(\varphi_{k+1}\right) \in W^{2, p_{1}}(\Omega)$. Hence, on account of the fact that $\nabla B\left(\varphi_{k+1}\right)=\lambda\left(\varphi_{k+1}\right) \nabla \varphi_{k+1}$, we further infer that $\nabla B\left(\varphi_{k+1}\right) \in W^{1, p_{1}}(\Omega)$ and $\nabla \varphi_{k+1} \in W^{1, p_{1}}(\Omega)$. Thus, by Sobolev embedding, we get an improved regularity for the convective term $\boldsymbol{U}_{k} \cdot \nabla \varphi_{k+1}$, which, by means of elliptic regularity 
again, implies that $B\left(\varphi_{k+1}\right) \in W^{2, p_{2}}(\Omega)$, with $1 / p_{2}=1 / p_{1}-1 / 3+1 / r$. By repeating this argument $n$ times, we get $B\left(\varphi_{k+1}\right) \in W^{2, p_{n}}(\Omega)$, where $1 / p_{n+1}=1 / p_{n}-1 / 3+1 / r$. This recursive relation can be made explicit by giving

$$
p_{n}=\frac{p_{1}}{1-(n-1) \sigma p_{1}}, \quad \sigma:=\frac{1}{3}-\frac{1}{r} .
$$

Therefore, after $n$ steps, with $n$ big enough, we have $p_{n} \geq 2$. The bootstrap argument then leads to $B\left(\varphi_{k+1}\right) \in H^{2}(\Omega)$ such that by (4.13), we also have $\varphi_{k+1} \in H^{2}(\Omega)$ (actually, one could also push the regularity for $\varphi_{k+1}$ further; however the $H^{2}$-regularity is enough for our purpose).

Let us now consider the discrete estimates that can be derived from the incremental-step problem (4.9)-(4.10). The basic estimate (4.17) still holds. As far as the estimates (4.18)-(4.20) and (4.22) are concerned, these can be repeated. However, the contribution coming from the convective term $\boldsymbol{U}_{k} \cdot \nabla \varphi_{k+1}$ in (4.22), instead of being estimated as in (4.23), is now controlled as follows (let us consider just the case $d=3$, and estimate only the main part of this contribution, recalling (4.5)):

$$
\begin{aligned}
& \tau \sum_{k=0}^{n}\left\|\boldsymbol{U}_{k} \cdot \nabla B\left(\varphi_{k+1}\right)\right\|^{2} \leq \tau \sum_{k=0}^{n}\left\|\boldsymbol{U}_{k}\right\|_{L^{r}(\Omega)^{3}}^{2}\left\|\nabla B\left(\varphi_{k+1}\right)\right\|_{L^{2 r /(r-2)}(\Omega)^{3}}^{2} \\
& \leq \tau \sum_{k=0}^{n}\left\|\boldsymbol{U}_{k}\right\|_{L^{r}(\Omega)^{3}}^{2}\left\|\nabla B\left(\varphi_{k+1}\right)\right\|^{2-\frac{6}{r}}\left\|\nabla B\left(\varphi_{k+1}\right)\right\|_{V}^{\frac{6}{r}} \\
& \leq \delta \tau \sum_{k=0}^{n}\left\|B\left(\varphi_{k+1}\right)\right\|_{H^{2}(\Omega)}^{2}+C_{\delta} \tau \sum_{k=0}^{n}\left\|\boldsymbol{U}_{k}\right\|_{L^{r}(\Omega)^{3}}^{\frac{2 r}{r-3}}\left\|\nabla B\left(\varphi_{k+1}\right)\right\|^{2}
\end{aligned}
$$

where $\delta>0$ is to be fixed later. Here, the Gagliardo-Nirenberg inequality has been used. It is easy to see that

$$
\tau \sum_{k=0}^{n}\left\|\boldsymbol{U}_{k}\right\|_{L^{r}(\Omega)^{3}}^{\frac{2 r}{r-3}} \leq\|\boldsymbol{u}\|_{L^{\frac{2 r}{r-3}}\left(0, T ; L^{r}(\Omega)^{3}\right)}^{\frac{2 r}{r-3}} .
$$

Therefore, taking (4.24)-(4.28) into account, from the discrete Gronwall's Lemma and from (6.1), (6.7), we can recover estimate (4.31) (the constant $\mathbb{Q}$ now depends on the norm of $\boldsymbol{u}$ on the righthand side of (6.7)). This allows us to deduce (6.2).

Next, as far as the regularity (6.3) is concerned, let us consider the two cases $d=2,3$ separately. In the case $d=2$, we can argue exactly as in Step 1 in the proof of Theorem 3.6, by using estimate (4.32), which can now be written in the form

$$
\tau \sum_{k=0}^{n}\left\|B\left(\varphi_{k+1}\right)\right\|_{H^{2}(\Omega)}^{2} \leq \mathbb{Q}\left(\left\|\varphi_{0}\right\|_{V},\|\boldsymbol{u}\|_{L^{2 r /(r-d)}\left(0, T ; L^{r}(\Omega)^{d}\right)}\right),
$$

and which is derived from (4.24), combined with (4.25)-(4.28), (4.31), (6.1), (6.6) and (6.7). If $d=3$, the argument requires some further care. The first step is to prove a bound in $L^{4}\left(0, T ; L^{4}(\Omega)^{3}\right)$ for the sequence of $\nabla B\left(\bar{\varphi}_{N}\right)$, namely,

$$
\tau \sum_{k=0}^{n}\left\|\nabla B\left(\varphi_{k+1}\right)\right\|_{L^{4}(\Omega)^{3}}^{4} \leq \mathbb{Q}\left(\left\|\varphi_{0}\right\|_{V},\|\boldsymbol{u}\|_{L^{2 r /(r-3)}\left(0, T ; L^{r}(\Omega)^{3}\right)}\right), \quad n=0, \ldots, N-1 .
$$

This bound is a consequence of (6.8) and of the following Gagliardo-Nirenberg inequality (which holds for every dimension $d$, see, e.g., $[17,18,41]$ )

$$
\left\|\nabla B\left(\varphi_{k+1}\right)\right\|_{L^{4}(\Omega)^{3}} \leq C\left\|B\left(\varphi_{k+1}\right)\right\|_{L^{\infty}(\Omega)}^{1 / 2}\left\|B\left(\varphi_{k+1}\right)\right\|_{H^{2}(\Omega)}^{1 / 2},
$$


provided that we prove a uniform bound in $L^{\infty}(\Omega)$ for the time discrete solutions $\varphi_{k+1}$ to the incremental-step problem (4.9)-(4.10), namely,

$$
\sup _{0 \leq k \leq n}\left\|\varphi_{k+1}\right\|_{L^{\infty}(\Omega)} \leq C\left(\left\|\varphi_{0}\right\|_{L^{\infty}(\Omega)}\right), \quad n=0, \ldots, N-1 .
$$

Once we have (6.11), we also find a bound for $\nabla \bar{\varphi}_{N}$ and for $\nabla \lambda\left(\bar{\varphi}_{N}\right)$ in $L^{4}\left(0, T ; L^{4}(\Omega)^{3}\right)$. Moreover, since we know that $\varphi_{k+1} \in H^{2}(\Omega)$, then (4.13) holds. From this identity, we deduce the bound for $\bar{\varphi}_{N}$ in $L^{2}\left(0, T ; H^{2}(\Omega)\right)$, which yields $(6.3)$.

Part (b). Let us prove (6.11). This is achieved through a Moser-Alikakos iteration argument performed on (4.9)-(4.10). Let us begin with an elementary identity that can be obtained from $2(a-b) a=a^{2}-b^{2}+(a-b)^{2}$, by multiplying it by $a^{2}$, then by multiplying the resulting identity by $a^{4}$, and iterating this procedure $j \geq 1$ times. We obtain that

$$
(a-b) a^{2^{j}-1}=\frac{1}{2^{j}} a^{2^{j}}-\frac{1}{2^{j}} b^{2^{j}}+A_{j}(a, b),
$$

where $A_{j}(a, b) \geq 0$ is some polynomial function of order $2^{j}$ which we do not write explicitly, since it is not essential. We now set $p_{j}:=2^{j}$, multiply (4.9) by $\varphi_{k+1}^{p_{j}-1}$, integrate over $\Omega$ (taking the boundary condition (4.10) and the incompressibility condition for $\boldsymbol{U}_{k}$ into account), and sum the resulting identity over $k$, for $k=0, \ldots, n$, with $0 \leq n \leq N-1$. By means of (6.12), we easily get the estimate

$$
\begin{aligned}
& \frac{1}{p_{j}} \int_{\Omega} \varphi_{n+1}^{p_{j}} d x+\frac{4 \alpha_{0}}{p_{j} p_{j}^{\prime}} \tau \sum_{k=0}^{n} \int_{\Omega}\left|\nabla\left(\varphi_{k+1}^{p_{j} / 2}\right)\right|^{2} d x \\
& \leq \frac{1}{p_{j}} \int_{\Omega} \varphi_{0}^{p_{j}} d x+\tau \sum_{k=0}^{n}\left(m\left(\varphi_{k}\right)\left(\nabla K * Q\left(\varphi_{k}\right)\right), \nabla\left(\varphi_{k+1}^{p_{j}-1}\right)\right),
\end{aligned}
$$

where $p_{j}^{\prime}$ is the conjugate exponent to $p_{j}$. Let us estimate the term on the right-hand side of (6.13). We have

$$
\begin{aligned}
& \tau \mid \sum_{k=0}^{n}\left(m\left(\varphi_{k}\right)\left(\nabla K * Q\left(\varphi_{k}\right)\right), \nabla\left(\varphi_{k+1}^{p_{j}-1}\right) \mid\right. \\
& \leq \frac{\alpha_{0}}{p_{j} p_{j}^{\prime}} \tau \sum_{k=0}^{n} \int_{\Omega}\left|\nabla\left(\varphi_{k+1}^{p_{j} / 2}\right)\right|^{2} d x+\frac{m_{\infty}^{2} b^{2}}{\alpha_{0} p_{j}^{\prime}} p_{j} \tau \sum_{k=0}^{n} \int_{\Omega}\left(\frac{1}{p_{j-1}^{\prime}}\left|\varphi_{k+1}^{p_{j} / 2}\right|^{2} d x+\frac{2}{p_{j}}\right) .
\end{aligned}
$$

By means of this estimate, and setting $\psi_{k}^{(j)}:=\varphi_{k}^{p_{j} / 2},(6.13)$ yields that

$$
\begin{aligned}
& \int_{\Omega}\left|\psi_{n+1}^{(j)}\right|^{2} d x+\frac{\alpha_{0}}{p_{j}^{\prime}} \tau \sum_{k=0}^{n} \int_{\Omega}\left|\nabla \psi_{k+1}^{(j)}\right|^{2} d x \\
& \leq \int_{\Omega}\left|\psi_{0}^{(j)}\right|^{2} d x+C p_{j}^{2} \tau \sum_{k=0}^{n} \int_{\Omega}\left|\psi_{k+1}^{(j)}\right|^{2} d x+C p_{j},
\end{aligned}
$$

where $C>0$ shall henceforth denote some positive constant which may depend on the mobility $m$, and on $K, \alpha_{0}, \Omega$ and $T$, but are independent of the index $j$ and of $N$. Using the following 3D Gagliardo-Nirenberg inequality,

$$
\left\|\psi_{k+1}^{(j)}\right\|^{2} \leq C\left(\left\|\psi_{k+1}^{(j)}\right\|_{L^{1}(\Omega)}^{4 / 5}\left\|\nabla \psi_{k+1}^{(j)}\right\|^{6 / 5}+\left\|\psi_{k+1}^{(j)}\right\|_{L^{1}(\Omega)}^{2}\right),
$$


and Young's inequality in (6.15), we obtain that

$$
\int_{\Omega}\left|\psi_{n+1}^{(j)}\right|^{2} d x+\frac{\alpha_{0}}{2 p_{j}^{\prime}} \tau \sum_{k=0}^{n} \int_{\Omega}\left|\nabla \psi_{k+1}^{(j)}\right|^{2} d x \leq \int_{\Omega}\left|\psi_{0}^{(j)}\right|^{2} d x+C p_{j}^{5} \tau \sum_{k=0}^{n}\left\|\psi_{k+1}^{(j)}\right\|_{L^{1}(\Omega)}^{2} .
$$

The last inequality implies that

$$
\begin{aligned}
& \int_{\Omega} \varphi_{n+1}^{p_{j}} d x \\
& \leq \int_{\Omega} \varphi_{0}^{p_{j}} d x+C p_{j}^{5} \tau \sum_{k=0}^{n}\left(\int_{\Omega}\left|\varphi_{k+1}\right|^{p_{j-1}} d x\right)^{2} \\
& \leq \int_{\Omega} \varphi_{0}^{p_{j}}+C p_{j}^{5} T \max _{0 \leq k \leq n}\left(\int_{\Omega}\left|\varphi_{k+1}\right|^{p_{j-1}} d x\right)^{2} \\
& \leq C p_{j}^{5}\left(\max _{0 \leq k \leq N-1}\left\{1, \int_{\Omega}\left|\varphi_{k+1}\right|^{p_{j-1}} d x\right\}\right)^{2},
\end{aligned}
$$

where we have used the fact that $\left\|\varphi_{0}\right\|_{L^{\infty}(\Omega)} \leq 1$, and where the constant $C>0$ depends on $\left\|\varphi_{0}\right\|_{L^{\infty}(\Omega)}$. Setting

$$
E_{j}:=\max _{0 \leq k \leq N-1}\left\{1, \int_{\Omega}\left|\varphi_{k+1}\right|^{p_{j}} d x\right\}, \quad \forall j \geq 0,
$$

we obtain from (6.17) the recursive relation

$$
E_{j} \leq C p_{j}^{5} E_{j-1}^{2}, \quad j \geq 1
$$

so that

$$
E_{j} \leq C^{\sum_{l=0}^{j-1} 2^{l}} \prod_{l=0}^{j-1} p_{j-l}^{5 \cdot 2^{l}} E_{0}^{2^{j}}
$$

Consequently,

$$
\begin{aligned}
\max _{0 \leq k \leq N-1}\left\|\varphi_{k+1}\right\|_{L^{p_{j}(\Omega)}} & \leq C 2^{5 \sum_{\ell=1}^{j} \frac{\ell}{2^{\ell}}} E_{0} \\
& \leq C \max _{0 \leq k \leq N-1}\left\{1, \int_{\Omega}\left|\varphi_{k+1}\right| d x\right\} \\
& \leq C\left(\left\|\varphi_{0}\right\|_{L^{\infty}(\Omega)}\right),
\end{aligned}
$$

where (4.17) has been taken into account in the last estimate. Letting $j \rightarrow \infty$, and using the fact that the constant $C$ depends neither on the index $j$ nor on $N$, we conclude (6.11) from (6.18).

Part (c). We now prove uniqueness of the strong solution satisfying (6.2)-(6.3). Let us start with the case $d=2$. We take the difference of (3.1) and (3.3), written for two solutions, and multiply the resulting identity by $\varphi:=\varphi_{2}-\varphi_{1}$ in $H$. We then get

$$
\begin{aligned}
& \frac{1}{2} \frac{d}{d t}\|\varphi\|^{2}+\left(\nabla\left(B\left(\varphi_{2}\right)-B\left(\varphi_{1}\right)\right), \nabla \varphi\right) \\
& =\left(\left(m\left(\varphi_{2}\right)-m\left(\varphi_{1}\right)\right)\left(\nabla K * \varphi_{2}\right), \nabla \varphi\right)+\left(m\left(\varphi_{1}\right)(\nabla K * \varphi), \nabla \varphi\right) .
\end{aligned}
$$

Thanks to (A4), we can deduce that

$$
\left(\nabla\left(B\left(\varphi_{2}\right)-B\left(\varphi_{1}\right)\right), \nabla \varphi\right) \geq \alpha_{0}\|\nabla \varphi\|^{2}+\left(\left(\lambda\left(\varphi_{2}\right)-\lambda\left(\varphi_{1}\right)\right) \nabla \varphi_{2}, \nabla \varphi\right),
$$


and, due to the regularity estimate $(6.2)$ for $\varphi_{2}$, we have that

$$
\begin{aligned}
& \left|\left(\left(\lambda\left(\varphi_{2}\right)-\lambda\left(\varphi_{1}\right)\right) \nabla \varphi_{2}, \nabla \varphi\right)\right| \\
& \leq C\left(\|\varphi\|+\|\varphi\|^{1 / 2}\|\nabla \varphi\|^{1 / 2}\right)\left\|\nabla \varphi_{2}\right\|^{1 / 2}\left\|\varphi_{2}\right\|_{H^{2}(\Omega)}^{1 / 2}\|\nabla \varphi\| \\
& \leq \frac{\alpha_{0}}{6}\|\nabla \varphi\|^{2}+C\left(1+\left\|\varphi_{2}\right\|_{H^{2}(\Omega)}^{2}\right)\|\varphi\|^{2} .
\end{aligned}
$$

The estimates of the two terms on the right-hand side of (6.19) being straightforward, we are led to

$$
\frac{d}{d t}\|\varphi\|^{2}+\alpha_{0}\|\nabla \varphi\|^{2} \leq C\left(1+\left\|\varphi_{2}\right\|_{H^{2}(\Omega)}^{2}\right)\|\varphi\|^{2}
$$

Uniqueness, and also a continuous dependence estimate, then follow from applying Gronwall's lemma, owing once again to (6.3) for $\varphi_{2}$.

For $d=3$, the test by $\varphi$ does not work for uniqueness (the difficulty lies in the estimate of the term $\left.\left(\left(\lambda\left(\varphi_{2}\right)-\lambda\left(\varphi_{1}\right)\right) \nabla \varphi_{2}, \nabla \varphi\right)\right)$. The test by $\left(-\Delta_{N}\right)^{-1} \varphi$ works $\left(-\Delta_{N}\right.$ being the Laplace operator with homogeneous Neumann boundary condition), provided that $\boldsymbol{u} \in L^{2}\left(0, T ; L^{\infty}(\Omega)^{3}\right)$. Uniqueness then follows by arguing as in [24, Proposition 4].

Part (d). Let us now prove the last part of the theorem. If $d=2$ then we can argue as in Step 3 of the proof of Theorem 3.6. Identity (4.67) and estimates (4.68)-(4.73) can be rewritten in such a way that the discrete inequality (4.75) holds, where the constant $C$ now depends on the norm of $\boldsymbol{u}$ on the right-hand side of (6.7). Also the argument for the control of $\left(\varphi_{1}-\varphi_{0}\right) / \tau$ in $L^{2}$ still works, with only one difference. More precisely, instead of (4.78), we now have, as a consequence of the first of (6.4),

$$
\left\|\boldsymbol{U}_{k}\right\|_{L^{\infty}(\Omega)^{2}} \leq \frac{1}{\tau^{1 / s}}\|\boldsymbol{u}\|_{L^{s}\left(0, T ; L^{\infty}(\Omega)^{2}\right)} .
$$

Hence, instead of using Agmon's inequality in (4.79), we deduce that

$$
\begin{aligned}
& \tau\left|\left(\boldsymbol{U}_{k} \cdot\left(\varphi_{2}-\varphi_{1}\right), \nabla\left(B\left(\varphi_{2}\right)-B\left(\varphi_{1}\right)\right)\right)\right| \\
& \leq \tau\left\|\boldsymbol{U}_{k}\right\|_{L^{\infty}(\Omega)^{2}}\left\|\varphi_{2}-\varphi_{1}\right\|\left\|\nabla\left(B\left(\varphi_{2}\right)-B\left(\varphi_{1}\right)\right)\right\| \\
& \leq \frac{\tau}{2}\left\|\nabla\left(B\left(\varphi_{2}\right)-B\left(\varphi_{1}\right)\right)\right\|^{2}+\frac{1}{2} \tau^{1-\frac{2}{s}}\|\boldsymbol{u}\|_{L^{s}\left(0, T ; L^{\infty}(\Omega)^{2}\right)}^{2}\left\|\varphi_{2}-\varphi_{1}\right\|^{2} .
\end{aligned}
$$

Since $s>2$, we can choose $0<\tau \leq \tau_{1}$, with $\tau_{1}$ small enough (and depending on the norm of $\boldsymbol{u}$ on the right-hand side of (6.7)), and still obtain (4.82), yielding the desired control for the quotient $\left(\varphi_{1}-\varphi_{0}\right) / \tau$. Owing to this control and to the second property of (6.4) and (4.84), we still get (4.85) from (4.75), which allows to obtain the second part of (6.5).

Finally, in order to deduce the first part of (6.5), we can argue as in Step 1 of the proof of Theorem 3.6, first estimating the $H^{2}$-norm of $B\left(\varphi_{k+1}\right)$ by elliptic regularity and then using (4.9) (cf. (4.24)). The $L^{2}$-norm of the advective term, which essentially amounts to control $\boldsymbol{U}_{k} \cdot \nabla B\left(\varphi_{k+1}\right)$, on account of the first of (6.4), can now be estimated as follows:

$$
\begin{aligned}
& \left\|\boldsymbol{U}_{k} \cdot \nabla B\left(\varphi_{k+1}\right)\right\| \leq\left\|\boldsymbol{U}_{k}\right\|_{L^{\sigma}(\Omega)^{2}}\left\|\nabla B\left(\varphi_{k+1}\right)\right\|_{L^{2 \sigma /(\sigma-2)}(\Omega)^{2}} \\
& \leq C\|\boldsymbol{u}\|_{L^{\infty}\left(0, T ; L^{\sigma}(\Omega)^{2}\right)}\left\|\nabla B\left(\varphi_{k+1}\right)\right\|^{1-2 / \sigma}\left\|B\left(\varphi_{k+1}\right)\right\|_{H^{2}(\Omega)}^{2 / \sigma} \\
& \leq \delta\left\|B\left(\varphi_{k+1}\right)\right\|_{H^{2}(\Omega)}+\mathbb{Q}_{\delta}\left(\left\|\varphi_{0}\right\|_{V},\|\boldsymbol{u}\|_{L^{\infty}\left(0, T ; L^{\sigma}(\Omega)^{2}\right)},\|\boldsymbol{u}\|_{L^{2 r /(r-2)}\left(0, T ; L^{r}(\Omega)^{2}\right)}\right) .
\end{aligned}
$$

Therefore, choosing $\delta>0$ small enough, we get

$$
\left\|B\left(\bar{\varphi}_{N}\right)\right\|_{H^{2}(\Omega)} \leq \mathbb{Q}\left(\left\|\varphi_{0}\right\|_{V},\|\boldsymbol{u}\|_{L^{\infty}\left(0, T ; L^{\sigma}(\Omega)^{2}\right) \cap L^{2 r /(r-2)}\left(0, T ; L^{r}(\Omega)^{2}\right)}\right)\left(\left\|\widehat{\varphi}_{N}^{\prime}\right\|+1\right) .
$$


Owing now to the uniform bound for $\widehat{\varphi}_{N}^{\prime} \in L^{\infty}(0, T ; H)$, the foregoing estimate yields a bound for $B\left(\bar{\varphi}_{N}\right)$ in $L^{\infty}\left(0, T ; H^{2}(\Omega)\right)$; henceforth, we also have

$$
\nabla B\left(\bar{\varphi}_{N}\right), \nabla \bar{\varphi}_{N}, \nabla \lambda\left(\bar{\varphi}_{N}\right) \in L^{\infty}\left(0, T ; L^{p}(\Omega)^{2}\right), \text { for all } p<\infty .
$$

Thus, on account of (4.13), we find the desired bound for $\bar{\varphi}_{N}$ in $L^{\infty}\left(0, T ; H^{2}(\Omega)\right)$. Hence, the first part of (6.5) is proven and the proof of the theorem is finished.

Remark 6.2. The bound (6.11) obviously also holds for $d=2$. Therefore, the argument relying on (6.10) can be employed, both in Theorem 3.6 and in Theorem 6.1 , to deduce the $L^{2}\left(0, T ; H^{2}(\Omega)\right)$ regularity for $\varphi$ in two dimensions as well. However, we point out that, in the case $d=2$, this regularity can be established without using (6.11).

Remark 6.3. If $d=3$ the regularity (6.5) is open unless we assume $\lambda:=m F^{\prime \prime}$ constant (in this case (6.4) is still required). It is worth observing that these assumptions are basically the ones considered in [31]) whose regularity was discussed in [39]. Moreover, if $\lambda$ is constant then uniqueness of the strong solution satisfying (6.2)-(6.3) holds for $d=3$, also under the more general condition (6.1) (without the need to assume $r=\infty$ ). Indeed, the second term on the right-hand side of (6.20) vanishes.

Analogous to Proposition 5.1, we may employ the uniform Gronwall's lemma (or, more precisely, its discrete variant, see [43, Lemma 3]), to establish uniform in time regularity estimates for the convective nonlocal Cahn-Hilliard equation with a prescribed (divergent-free) velocity. We can therefore deduce from Theorem 6.1 another result obtained by working with translation bounded functions and providing also a dissipative estimate for $\varphi$ (cf. (5.5)). We omit the statement of this theorem and its proof, since they can be deduced in a straightforward way. Moreover (cf. Remark $6.3)$, if $d=3$ and

$$
m F^{\prime \prime}=\lambda_{0}\left(\lambda_{0} \text { is a positive constant }\right),
$$

then it follows that $\varphi \in L^{\infty}\left(\mathbb{R}_{+} ; H^{2}(\Omega)\right)$ and $\varphi_{t} \in L^{\infty}\left(\mathbb{R}_{+} ; H\right) \cap L_{t b}^{2}\left(\mathbb{R}_{+} ; V\right)$, provided $\varphi_{0} \in H^{2}(\Omega)$ satisfies (3.11) and $\boldsymbol{u}$ satisfies (6.4) in the corresponding translation bounded spaces.

As far as the time continuity property of (5.40) is concerned, assume that all the conditions of Theorem 6.1 and, in addition, suppose that (M1b), (A1b) are fulfilled. By arguing as in the second part of Remark 5.2, we can easily see that the second of (5.40) still holds, under the further regularity $\boldsymbol{u} \in C^{0}\left([0, T] ; L^{\sigma}(\Omega)^{d}\right)$, for some $\sigma>d$, and, if $d=3$, provided that (6.21) holds. Suppose now that assumptions (K), (M), (A1)-(A4) are satisfied and that $\boldsymbol{u} \in L^{\infty}(\Omega)^{d}$ is independent of time. Then we know from [24, Section 6] that (1.2), (1.3), (1.5) 2 and (1.6) $)_{2}$ generates a semigroup of closed operators $\left\{S_{\kappa}(t)\right\}_{t \geq 0}$, with $\kappa \in[0,1]$ fixed, on the phase space $\mathcal{Y}_{\kappa}$, defined as in (5.42) and endowed with the metric induced by the $L^{2}$-norm. Namely, $\varphi \in C^{0}\left([0, \infty), \mathcal{Y}_{\kappa}\right)$ given by $\varphi(t):=S_{\kappa}(t) \varphi_{0}$, for all $t \geq 0$, is the (unique) weak solution to (1.2), (1.3), (1.5) 2 and (1.6) 2 corresponding to $\varphi_{0} \in \mathcal{Y}_{\kappa}$. According to [24, Theorem 5], this semigroup possesses a connected global attractor $\widetilde{\mathcal{A}}_{\kappa}$.

Assume now, in addition, that the (M1b) and (A1t) are fulfilled, and, for $d=3$, that (6.21) holds. It is then easy to check that the argument devised at the end of Section 5 to prove the regularity of the global attractor for (1.2)-(1.6), can be adapted to the present situation. This yields the following result.

Theorem 6.4. Suppose that assumptions $(\boldsymbol{K}),(\boldsymbol{M} \mathbf{1 b}),(\boldsymbol{A} \mathbf{1 t}),(\boldsymbol{A 4})$ are satisfied, that $K \in$ $W_{\text {loc }}^{2,1}\left(\mathbb{R}^{d}\right)$ or that $K$ is admissible, and that $\boldsymbol{u} \in L^{\infty}(\Omega)^{d}, d=2,3$, is independent of time. Moreover, if $d=3$, assume that (6.21) holds. Then the global attractor $\widetilde{\mathcal{A}}_{k}$ of the dynamical system 
$\left(\mathcal{Y}_{k},\left\{S_{\kappa}(t)\right\}_{t \geq 0}\right)$ generated by (1.2), (1.3), (1.5) $2,(1.6)_{2}$ is such that

$$
\widetilde{\mathcal{A}}_{\kappa} \subset \mathcal{B}_{\mathcal{Z}_{\kappa}}(\Lambda(k)) \text {, }
$$

where $\mathcal{B}_{\mathcal{Z}_{\kappa}}(\Lambda(k))$ is the closed ball in the metric space $\mathcal{Z}_{k}$ (cf. (5.43)), endowed with the metric induced by the $H^{2}$-norm, having radius $\Lambda(k)$, for some $\Lambda(k)>0$.

Acknowledgments. The first and third authors are members of the Gruppo Nazionale per l'Analisi Matematica, la Probabilità e le loro Applicazioni (GNAMPA) of the Istituto Nazionale di Alta Matematica (INdAM). The first author is "titolare di un Assegno di Ricerca dell'Istituto Nazionale di Alta Matematica".

\section{REFERENCES}

[1] H. Abels, On a diffusive interface model for two-phase flows of viscous, incompressible fluids with matched densities, Arch. Ration. Mech. Anal. 194 (2009), 463-506.

[2] H. Abels, Longtime behavior of solutions of a Navier-Stokes/Cahn-Hilliard system, Proceedings of the Conference "Nonlocal and Abstract Parabolic Equations and their Applications", Bedlewo, Banach Center Publ. 86 (2009), 9-19.

[3] H. Abels, D. Depner, H. Garcke, On an incompressible NavierStokes/Cahn Hilliard system with degenerate mobility, Ann. Inst. H. Poincaré Anal. Non Linéaire 30 (2013), 1175-1190.

[4] H Abels, Y. Terasawa, On Stokes operators with variable viscosity in bounded and unbounded domains, Math. Ann. 344 (2009), 381-429.

[5] S. Agmon, Lectures on elliptic boundary value problems, Mathematical Studies, Van Nostrand, New York, 1965.

[6] J.M. Ball, Continuity properties and global attractors of generalized semiflows and the Navier-Stokes equation, J. Nonlinear Sci. 7 (1997), 475-502 (Erratum, J. Nonlinear Sci. 8 (1998), 233).

[7] S. Bastea, R. Esposito, J.L. Lebowitz, R. Marra, Sharp interface motion of a binary fluid mixture, J. Stat. Phys. 124 (2006), 445-483.

[8] P.W. Bates, J. Han, The Neumann boundary problem for a nonlocal Cahn-Hilliard equation, J. Differential Equations 212 (2005), 235-277.

[9] J. Bedrossian, N. Rodríguez, A. Bertozzi, Local and global well-posedness for an aggregation equation and PatlakKeller-Segel models with degenerate diffusion, Nonlinearity 24 (2011), 1683-1714.

[10] O.V. Besov, V.P. Il'in, S.M. Nikol'skiǔ, Integral representations of functions and embedding theorems. Vol. II, Scripta Series in Mathematics. Edited by M.H. Taibleson. V. H. Winston \& Sons, Washington, D.C.; Halsted Press [John Wiley \& Sons], New York-Toronto, Ont.-London, 1979.

[11] H. Brézis, Équations et inéquations non-linéaires dans les espaces vectoriel en dualité, Ann. Inst. Fourier 18 (1968), 115-176.

[12] F. Boyer, Mathematical study of multi-phase flow under shear through order parameter formulation, Asymptot. Anal. 20 (1999), 175-212.

[13] C. Cao, C.G. Gal, Global solutions for the $2 D$ NS-CH model for a two-phase flow of viscous, incompressible fluids with mixed partial viscosity and mobility, Nonlinearity 25 (2012), 3211-3234.

[14] P. Colli, S. Frigeri, M. Grasselli, Global existence of weak solutions to a nonlocal Cahn-Hilliard-Navier-Stokes system, J. Math. Anal. Appl. 386 (2012), 428-444.

[15] E. DiBenedetto, Real Analysis, Birkhäuser, Boston, Advanced Text Series, 2002.

[16] C.M. Elliott, H. Garcke, On the Cahn-Hilliard equation with degenerate mobility, SIAM J. Math. Anal. 27 (1996), 404-423.

[17] A. Friedman, Partial differential equations, Holt, Rinehart and Winston, New York, 1969.

[18] E. Gagliardo, Ulteriori proprietà di alcune classi di funzioni in più variabili, Ricerche Mat. 8 (1959), $24-51$.

[19] S. Frigeri, Global existence of weak solutions for a nonlocal model for two-phase flows of incompressible fluids with unmatched densities, Math. Models Methods Appl. Sci. 26 (2016), 1955-1993.

[20] S. Frigeri, C.G. Gal, M. Grasselli On nonlocal Cahn-Hilliard-Navier-Stokes systems in two dimensions, J. Nonlinear Sci. 26 (2016), 847-893.

[21] S. Frigeri, M. Grasselli, Global and trajectories attractors for a nonlocal Cahn-Hilliard-Navier-Stokes system, J. Dynam. Differential Equations 24 (2012), 827-856.

[22] S. Frigeri, M. Grasselli, Nonlocal Cahn-Hilliard-Navier-Stokes systems with singular potentials, Dyn. Partial Differ. Equ. 9 (2012), 273-304. 
[23] S. Frigeri, M. Grasselli, P. Krejčí, Strong solutions for two-dimensional nonlocal Cahn-Hilliard-Navier-Stokes systems, J. Differential Equations 255 (2013), 2597-2614.

[24] S. Frigeri, M. Grasselli, E. Rocca, A diffuse interface model for two-phase incompressible flows with nonlocal interactions and nonconstant mobility, Nonlinearity 28 (2015), 1257-1293.

[25] S. Frigeri, E. Rocca, J. Sprekels, Optimal distributed control of a nonlocal Cahn-Hilliard/Navier-Stokes system in 2D, SIAM J. Control Optim. 54 (2016), 221-250.

[26] C.G. Gal, On an inviscid model for incompressible two-phase flows with nonlocal interaction, J. Math. Fluid Mechanics (2016), 18, 659-677.

[27] C.G. Gal, M. Grasselli, Asymptotic behavior of a Cahn-Hilliard-Navier-Stokes system in 2D, Ann. Inst. H. Poincaré Anal. Non Linéaire 27 (2010), 401-436.

[28] C.G. Gal, M. Grasselli, Trajectory attractors for binary fluid mixtures in 3D, Chinese Ann. Math. Ser. B 31 (2010), 655-678.

[29] C.G. Gal, M. Grasselli, Instability of two-phase flows: a lower bound on the dimension of the global attractor of the Cahn-Hilliard-Navier-Stokes system, Phys. D 240 (2011), 629-635.

[30] C.G. Gal, M. Grasselli, Longtime behavior of nonlocal Cahn-Hilliard equations, Discrete Contin. Dyn. Syst. Ser. A 34 (2014), 145-179.

[31] H. Gajewski, K. Zacharias, On a nonlocal phase separation model, J. Math. Anal. Appl. 286 (2003), 11-31.

[32] G. Giacomin, J.L. Lebowitz, Exact macroscopic description of phase segregation in model alloys with long range interactions, Phys. Rev. Lett. 76 (1996), 1094-1097.

[33] G. Giacomin, J.L. Lebowitz, Phase segregation dynamics in particle systems with long range interactions. I. Macroscopic limits, J. Statist. Phys. 87 (1997), 37-61.

[34] G. Giacomin, J.L. Lebowitz, Phase segregation dynamics in particle systems with long range interactions. II. Phase motion, SIAM J. Appl. Math. 58 (1998), 1707-1729.

[35] C.G. Gal, A. Giorgini, M. Grasselli, The nonlocal Cahn-Hilliard equation with singular potential: well-posedness, regularity and strict separation property, submitted.

[36] J.S. Kim, Phase-field models for multi-component fluid flows, Commun. Comput. Phys. 12 (2012), 613-661.

[37] C. Liu, J. Shen, A phase field model for the mixture of two incompressible fluids and its approximation by a Fourier spectral method, Phys. D 179 (2003), 211-228.

[38] S.-O. Londen, H. Petzeltová, Convergence of solutions of a non-local phase-field system, Discrete Contin. Dyn. Syst. Ser. S 4 (2011), 653-670.

[39] S.-O. Londen, H. Petzeltová, Regularity and separation from potential barriers for a non-local phase-field system, J. Math. Anal. Appl. 379 (2011), 724-735.

[40] O.A. Ladyženskaja, V.A. Solonnikov, N.N. Ural'ceva, Linear and quasilinear equations of parabolic type, AMS Transl. Monographs 23, AMS, Providence, R.I. 1968.

[41] L. Nirenberg, On elliptic partial differential equations, Ann. Scuola Norm. Sup. Pisa 13 (1959), $115-162$.

[42] T. Roubíček, Nonlinear Partial Differential Equations with Applications, Birkhäuser, 2005.

[43] J. Shen, Long time stabilities and convergences for the fully discrete nonliner Galerkin method, Appl. Anal. 38 (1990), 201-229.

[44] V.N. Starovoitov, The dynamics of a two-component fluid in the presence of capillary forces, Math. Notes 62 (1997), 244-254.

[45] Y. Sun, Z. Zhang, Global regularity for the initial-boundary value problem of the 2-D Boussinesq system with variable viscosity and thermal diffusivity, J. Differential Equations 255 (2013), 1069-1085.

[46] R. Temam, Navier-Stokes equations and nonlinear functional analysis, Second edition, CBMS-NSF Reg. Conf. Ser. Appl. Math. 66, SIAM, Philadelphia, PA, 1995.

[47] L. Zhao, H. Wu, H. Huang, Convergence to equilibrium for a phase-field model for the mixture of two viscous incompressible fluids, Commun. Math. Sci. 7 (2009), 939-962. 
Dipartimento di Matematica "N. Tartaglia”, Università Cattolica del Sacro Cuore (Brescia), Via Musei 41, Brescia I-25121, ItALY

E-mail address: sergiopietro.frigeri@unicatt.it, sergio.frigeri.sf@gmail.com

Florida International University, Department of Mathematics, Miami, FL 33199, USA

E-mail address: cgal@fiu.edu

Dipartimento di Matematica, Politecnico di Milano, Milano I-20133, Italy

E-mail address: maurizio.grasselli@polimi.it

Weierstrass Institute for Applied Analysis and Stochastics, Mohrenstr. 39, D-10117 Berlin, Germany, and Department of Mathematics, Humboldt-Universität Zu Berlin, Unter den Linden 6, D-10099 Berlin, Germany

E-mail address: juergen.sprekels@wias-berlin.de 